\title{
Experimentelle und numerische Untersuchungen zur \\ Ausbreitung von Volumenstörungen in thermischen Plumes
}

\author{
Dissertation \\ zur Erlangung des Doktorgrades \\ der Mathematisch-Naturwissenschaftlichen Fakultäten \\ der Georg-August-Universität zu Göttingen
}

vorgelegt von

Nils Laudenbach

aus

Bremen

Göttingen 2001 
D 7

Referent: Prof. Dr. U. Christensen

Korreferent: Prof. Dr. H. Eckelmann

Tag der mündlichen Prüfung: 14.12.2001 


\section{Inhaltsverzeichnis}

\begin{tabular}{ll}
\hline Abstract & 1
\end{tabular}

1 Einleitung 3

2 Experiment und Meßmethoden 9

2.1 Aufbau des Plumeexperiments . . . . . . . . . . . . . . . . . . 9 9

2.2 Temperaturmeßmethode . . . . . . . . . . . . . . . . . . . 12

2.2 .1 Theorie . . . . . . . . . . . . . . . . . . . . 12

2.2 .2 Aufbau . . . . . . . . . . . . . . . . . . . . 14

2.2.3 Auswertung und Beispielergebnisse . . . . . . . . . . . . 17

2.3 Particle Image Velocimetry . . . . . . . . . . . . . . . . . . . . . . . 19

2.3.1 Aufbau, Prinzip und Auswertungsmethode . . . . . . . . . . . . . 19

2.3.2 Experiment und Beispielergebnisse . . . . . . . . . . . . 23

3 Numerische Modellierung 27

3.1 Physikalische Grundgleichungen . . . . . . . . . . . . . . . . . 28

$3.1 .1 \quad$ Kontinuitäts- und Stokes-Gleichung . . . . . . . . . . . . . . 28

3.1 .2 Wärmetransportgleichung . . . . . . . . . . . . . . . 32

3.2 Anfangs- und Randbedingungen . . . . . . . . . . . . . . . . 32

3.2 .1 Unterseite . . . . . . . . . . . . . . . . . . . . 33

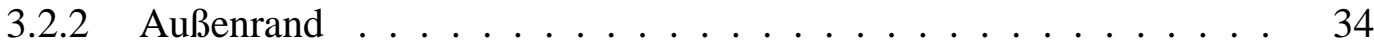

3.2 .3 Oberseite . . . . . . . . . . . . . . . . . . 34

3.2 .4 Zylinderachse. . . . . . . . . . . . . . . . . . . 35

3.2 .5 Temperaturanfangsbedingung . . . . . . . . . . . . 35

3.3 Diskretisierung und Lösungsverfahren . . . . . . . . . . . . . . . . . . 35

3.3 .1 Stokes-Gleichung. . . . . . . . . . . . . . . . . . . . . 36

3.3 .2 Wärmetransportgleichung . . . . . . . . . . . . . . . 38

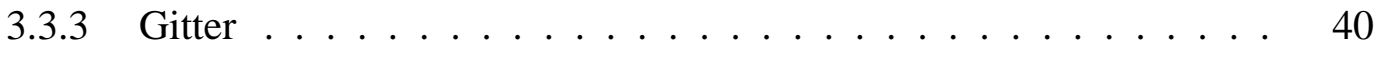

3.4 Programmtests . . . . . . . . . . . . . . . . 42

4 Beispielergebnisse und Vergleiche zwischen Numerik und Experiment 45

$4.1 \quad$ Stationäre Plumes . . . . . . . . . . . . . . . . . . 45 


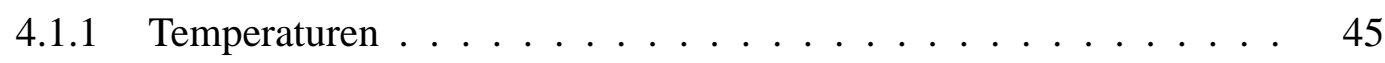

4.1.2 Geschwindigkeiten . . . . . . . . . . . . . . . . . . . . . 48

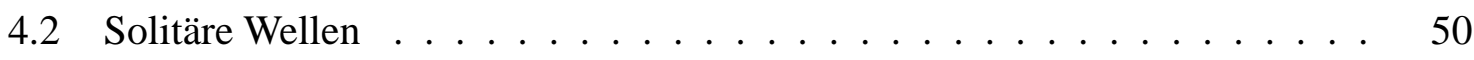

4.2 .1 Temperaturen . . . . . . . . . . . . . . . 51

4.2.2 Geschwindigkeiten . . . . . . . . . . . . . 56

5 Ergebnisse numerischer Parameterstudien

5.1 Wellengeschwindigkeit . . . . . . . . . . . . . . . . 65

5.2 Temperaturanomalie der Wellen . . . . . . . . . . . . . . . . . . 67

5.3 Einfluß einer tiefenabhängigen Viskosität . . . . . . . . . . . . . . . . . . 72

5.4 Skalierung auf die Mantelplumes der Erde . . . . . . . . . . . . . . 76

\begin{tabular}{lll}
\hline Diskussion & 79
\end{tabular}

$7 \begin{array}{lr}\text { Zusammenfassung } & 83\end{array}$

\begin{tabular}{|l|l}
\hline Anhang & $\mathbf{8 7}$
\end{tabular}

A.1 Sirupeigenschaften . . . . . . . . . . . . . . . . . . . . . . . . 88

A.1.1 Dichte, Viskosität und andere Größen . . . . . . . . . . . . . . . . . . . 89

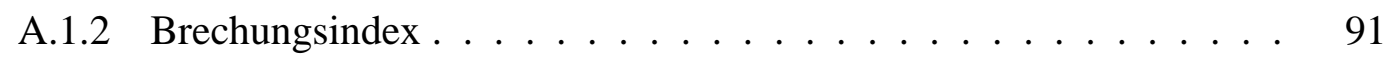

A.2 Gleichungen. . . . . . . . . . . . . . . . . . . . . . . . . . . . . . .

A.2.1 Stokesgleichung in Stromfunktionsformulierung . . . . . . . . . . . 92

A.2.2 Diskretisierung der Wärmetransportgleichung . . . . . . . . . . . . . 92

A.3 Verwendete Skalen . . . . . . . . . . . . . . . . . . . . . . . . . . . . . . . . . . . .

A.4 Verwendete Geräte . . . . . . . . . . . . . . . . . . . . . . . . . . . . 95

A.4.1 Nd:YAG-Laser . . . . . . . . . . . . . . . . . . . . . . . . . . . . 95

A.4.2 CCD-Kamera für PIV . . . . . . . . . . . . . . . . . . 95

A.4.3 HeNe-Laser für Temperaturmeßmethode . . . . . . . . . . . . . 95

A.4.4 CCD-Kamera für Temperaturmeßmethode . . . . . . . . . . . . 95

A.4.5 Galvanoscanner. . . . . . . . . . . . . . . 96

\begin{tabular}{|l|l}
\hline Literaturverzeichnis & 97
\end{tabular}

\begin{tabular}{ll}
\hline Danksagung & 101
\end{tabular} 


\section{Abbildungsverzeichnis}

2.1 Versuchsaufbau des Plumeexperiments . . . . . . . . . . . . . . . 10

2.2 Fotografische Aufnahme eines Diapirs und eines stationären Kanals . . . . 11

2.3 Fotografische Aufnahme einer solitären Welle . . . . . . . . . . . . 11

2.4 Skizze der Laserstrahlablenkung . . . . . . . . . . . . . . . . . . . . . 13

2.5 Temperatur und Ablenkungskurve für einen hypothetischen Plume . . . . . 14

2.6 Experimenteller Aufbau der Temperaturmeßmethode . . . . . . . . . . . 15

2.7 Exemplarische Ablenkungskurve und Vergleich der durch Linsenfehler verursachten Meßfehler . . . . . . . . . . . . . . . . . 16

2.8 Ablenkungskurven in verschiedenen Aufstiegshöhen und die daraus bestimmten Temperaturprofile . . . . . . . . . . . . . . . . . . . 17

2.9 Vollständig ausgemessene Temperaturfelder von zwei unterschiedlich starken Plumekanälen . . . . . . . . . . . . . . . . . . . . 19

2.10 Aufbau der PIV-Methode . . . . . . . . . . . . . . . . . . . . . 20

2.11 Schematische Darstellung einer PIV-Auswertung . . . . . . . . . . . 21

2.12 Beispielabtastflecken mit Korrelationsfunktion . . . . . . . . . . . . . 22

2.13 Horizontales Profil der Vertikalgeschwindigkeit in einem Plumekanal . . . 24

2.14 Gemessene Geschwindigkeiten in einem Diapir . . . . . . . . . . . . 25

3.1 Temperaturrandbedingung und vorgegebene Vertikalgeschwindigkeit. . . . 33

3.2 Differenzenschema . . . . . . . . . . . . . . . . . . 37

3.3 Besetzung der Bandmatrix . . . . . . . . . . . . . . . . . 37

3.4 ADI-Schema . . . . . . . . . . . . . . . . . . . . . . . . . . . 39

3.5 Nichtäquidistantes Gitter in radialer Richtung . . . . . . . . . . . . . 41

3.6 Vergleichsrechnungen zur Gitterauflösung . . . . . . . . . . . . . . . 41

3.7 Test mit analytisch berechneter Stromfunktion . . . . . . . . . . . . . . . 42

3.8 Vergleich der Zeitentwicklung zwischen CMG und CFDC . . . . . . . . 43

3.9 Vergleich von vertikalen Temperaturprofilen zwischen CMG und CFDC . . 44

3.10 Vergleich von radialen Temperaturprofilen zwischen CMG und CFDC . . . 44

4.1 Temperaturfeld eines aufsteigenden Diapirs und der dazugehörigen Kanalströmung (numerisch) . . . . . . . . . . . . . . . . . 46

4.2 Vergleich von experimentellen und numerischen Temperaturprofilen . . . . 47 
4.3 Vergleich zwischen einfachen Geschwindigkeitsmessungen und Numerik .

4.4 Vergleich zwischen einem gemessenen und einem numerisch bestimmten vertikalen Geschwindigkeitsfeld

4.5 Vergleich zwischen gemessenen und numerisch bestimmten vertikalen Geschwindigkeitsprofilen . . . . . . . . . . . . . . .

4.6 Vergleich zwischen experimentell und numerisch bestimmten Temperaturprofilen in einer aufsteigenden Welle . . . . . . . . . . . . . . . . .

4.7 Vergleich von experimentellen und numerischen Wellentemperaturen. . . . .

4.8 Vollständiges experimentell bestimmtes Temperaturfeld und Temperaturanomalie für eine aufsteigende Welle . . . . . . . . . . . . . 53

4.9 Temperaturanomalie einer aufsteigenden Welle . . . . . . . . . . . . 54

4.10 Differenztemperaturfelder zweier Wellen, erzeugt durch unterschiedliche lange Störungen . . . . . . . . . . . . . . . . 55

4.11 Numerisch bestimmte Wellengeschwindigkeiten . . . . . . . . . . . 57

4.12 Geschwindigkeitsstruktur einer aufsteigenden Welle (numerisch) . . . . . . 58

4.13 Vergleich zwischen einem gemessenen und einem numerisch bestimmten vertikalen Geschwindigkeitsfeld in einer aufsteigenden Welle . . . . . . . 59

4.14 Temperaturanomalien zweier gleicher hintereinander aufsteigender Wellen . 60

4.15 Vergleich zwischen einem gemessenen und einem numerisch bestimmten radialen Geschwindigkeitsfeld in einer aufsteigenden Welle . . . . . . . . . 61

4.16 Wechselwirkung zwischen zwei solitären Wellen in einem chemischen Plume 62

4.17 Kollision zwischen zwei unterschiedlich großen thermischen Wellen . . . . 63

5.1 Ausbreitungsgeschwindigkeit gegen Wellenvolumen (dimensionslos). . . . 66

5.2 Mit $Q /$ א skalierte Kanaltemperaturen . . . . . . . . . . . . . . 68

5.3 Verhältnis zwischen maximalen Wellen- und Kanaltemperaturen . . . . . . 69

5.4 Verhältnis zwischen maximalen Wellen- und Kanaltemperaturen in Abhängigkeit von der Aufstiegshöhe

5.5 Maximale Temperaturanomalie verschiedener Wellen gegenüber dimensionslosem Wellenvolumen ................. 70

5.6 Kanaltemperaturen bei tiefenabhängiger Viskosität . . . . . . . . . . . . . 72

5.7 Kanalgeschwindigkeit bei tiefenabhängiger Viskosität. . . . . . . . . . . . 73

5.8 Einfluß der tiefenabhängigen Viskosität auf den Plumeaufstieg . . . . . . . 74

5.9 Einfluß der tiefenabhängigen Viskosität auf den Wellenaufstieg . . . . . . . 75

5.10 Wellentemperaturen bei tiefenabhängiger Viskosität . . . . . . . . . . 76

6.1 Schmelzkurve für trockenen Peridotit . . . . . . . . . . . . 80

A.1 Temperaturabhängigkeit der Sirupdichte . . . . . . . . . . . . . . . . 89

A.2 Temperaturabhängigkeit der Sirupviskosität . . . . . . . . . . . . . . . . . . . . . 90

A.3 Temperaturabhängigkeit des Sirupbrechungsindex . . . . . . . . . . . . 91

A.4 Skizze einer niedrigviskosen Kanalströmung in einer hochviskosen Matrix. 93 


\section{Abstract}

The propagation of wavelike disturbances in thermal plumes conduit is studied in laboratory experiments and numerical simulations. In experiments thermal plumes are created by injecting hot corn syrup into a column of cold syrup. Wavelike disturbances are generated by enhancing the injection rate for a few seconds. For the measurement of the thermal structure of the plume a newly implemented non-intrusive method is used. It is based on the deflection of a laser beam passing through the plume. Continuous scanning provides a new radial temperature profile each second, which allows detailed studies of the thermal structure of solitary waves. Velocity measurements are performed with a PIV (Particle Image Velocimetry) - method.

For numerical simulation a finite difference code for cylindrical axisymmetric geometry was developed, based on a direct solver for the Stokes-equation and an ADI scheme for advancing temperature.

For the first time, a quantitative comparison between laboratory thermal plumes and conduit waves with a numerical model is presented. The comparison shows an excellent agreement between experimental and numerical temperature profiles of plume conduits and waves. Also the agreement for the velocities in the plume conduit is very good.

Studies of waves with different generation parameters show that the thermal structure and the propagation speed do not depend on the time and amplitude of disturbances but on the wave volume. Scaling laws are derived from the numerical calculations in order to describe the dependence of the propagation speed and wave temperature on the model parameters.

Scaling the results to the earth shows that waves are unlikely to exist in mantle plumes originating from the $660 \mathrm{~km}$ discontinuity, because the observed minimum wavelength is about $400 \mathrm{~km}$.

For mantle plumes with high buoyancy fluxes the results show only minor influence of waves with realistic wavelength on the temperature anomalies of the conduit. Significant increase of melt production is only expected for plumes with lower buoyancy fluxes under an older lithosphere. If the conduit is at the same temperature as the solidus, a temperature increase caused by an arriving wave yields a significant amount of partial melting. This can be a possible explanation for episodic magma production at weak hot-spots. 


\section{Kapitel 1}

\section{Einleitung}

Auf der Erde findet man neben dem Vulkanismus an Subduktionszonen und Mittelozeanischen Rücken auch vulkanisch aktive, lokal begrenzte Gebiete, die durch Bereiche erhöhter Temperatur unterhalb der Lithosphäre erzeugt werden. Offenbar unbeeinflußt von großräumigen Kontinentalplattenbewegungen verändern diese sogenannten „Hotspots“ über Zeitskalen von Millionen Jahren ihre Lage zueinander nicht oder nur geringfügig. Sie erzeugen auf bewegten Lithosphärenplatten Vulkanketten, in denen sich der jüngste Vulkanismus in unmittelbarer Nähe und der älteste am weitesten vom Hotspot entfernt befindet. In diesen Gebieten beobachtet man neben einem erhöhten Wärmefluß auch positive Geoidanomalien und topographische Aufwölbungen der Lithosphärenplatten, sowie geochemische Signaturen der Basaltlaven, die sich zum Teil stark von denen an Subduktionszonen und an Mittelozeanischen Rücken unterscheiden. Als Ursache für die Hotspots wurden von Morgan [1972] die sogenannten Mantelplumes (kurz: Plumes) vorgeschlagen. Dabei handelt es sich um heiße, säulenförmige, lokal begrenzte Aufströme im Erdmantel, die heißes Material aus großen Tiefen unter die Lithosphäre transportieren und so den beobachteten Vulkanismus sowie die Topographie- und Geoidanomalien erzeugen. Die unterschiedlichen chemischen Signaturen deuten auf unterschiedliche Quellregionen des aufgeschmolzenen Mantelmaterials hin.

Man geht davon aus, daß Plumes durch Instabilitäten in einer thermischen Grenzschicht entstehen und in einiger Entfernung zu dieser und der Lithosphäre eine angenähert axialsymmetrische Struktur aufweisen. Bei neu entstehenden Plumes vermutet man, daß sich zunächst ein großer pilzkopfartiger Plumekopf, ein sogenannter Diapir, aus heißem Material bildet. Dieser erfährt durch die thermische Ausdehnung des heißen Gesteins einen Auftrieb gegenüber dem Umgebungsmaterial, so daß der er langsam aufsteigt und weiteres heißes Material aus der Grenzschicht in einer dünnen kanalartigen Strömung nachfließen kann. Erreicht ein Diapir die Unterseite der Lithosphäre, so ist es möglich, daß der Solidus des Mantelgesteins weiträumig überschritten wird und große Mengen an Schmelzen produziert werden. Vermutlich sind die großen Flutbasaltregionen der Erde auf diese Weise entstanden (Duncan und Richards, [1991]). Abschätzungen aus der Schmelzenproduktion des beobachteten Vulkanismus haben ergeben, daß die Temperaturanomalie eines Plumes unterhalb der Lithosphäre im Vergleich zum Umgebungsmaterial etwa 200-300 K beträgt (Watson und 
McKenzie, [1991], White, [1993]). Die Aufstiegsgeschwindigkeiten in einem Plumekanal werden im allgemeinen mit etwa 10-100 cm/a angegeben (Morgan, [1972]). Sie sind damit etwa eine Größenordnung schneller als die für die großräumige Mantelkonvektion aus Plattenbewegungen abgeschätzten Geschwindigkeiten.

In der Geophysik sind Plumes im Laufe der Jahre zu einer größtenteils anerkannten Erklärung für Hotspots geworden. Dazu beigetragen haben auch die Ergebnisse der Seismologie (z.B. Wolfe et al., [1997], Li et al., [2000] und Ritter et al., [2001]), die für den oberen Mantel deutliche lokale seismische Geschwindigkeitsanomalien finden, die mit Plumes in Verbindung gebracht werden können. Obwohl diese seismologischen Erfolge kaum noch an der Existenz von Plumes zweifeln lassen, konnte ihre Quellregion bislang noch nicht eindeutig geklärt werden. Shen et al. [1998] finden unter Island eine um 20 km verringerte Übergangszonenmächtigkeit, was von den Autoren auf einen Plume mit einer Quellregion im unteren Mantel zurückgeführt wird. Olson [1990] vermutet, daß Plumes im unteren Mantel eventuell an der D"-Schicht durch Instabilitäten an der thermischen Grenzschicht zwischen Erdkern und Erdmantel entstehen. Diskutiert werden aber auch Quellregionen im mittleren Mantel (Kellog et al., [1999]) in ca. $1900 \mathrm{~km}$ und in der Übergangszone zwischen $410 \mathrm{~km}$ und $660 \mathrm{~km}$ Tiefe. Albers und Christensen [1996] zeigen in einem vereinfachten numerischen Modell, daß zumindest schwache Plumes aufgrund ihrer thermischen Auskühlung nicht von der Kern-Mantel-Grenze stammen können, sondern ihren Ursprung in geringeren Tiefen haben müssen.

Mit Hilfe der genannten und unzähliger weiterer geodynamischer Modellierungen versucht man die Physik des Erdmantels im allgemeinen (z.B. Christensen und Harder, [1990], Bunge et al., [1996]), sowie das Verhalten von abtauchenden Lithosphärenplatten (z.B. Christensen, [1997]) und aufsteigenden Plumes im speziellen (z.B. Olson et al., [1993]) genauer $\mathrm{zu}$ verstehen. Alle diese Modelle beruhen auf einem kontinuumsmechanischen Ansatz, mit dessen Hilfe man die Strömungsvorgänge im Erdmantel in den Grundgleichungen der Fluiddynamik beschreibt (Turcotte und Schubert, [1982]) oder mit Flüssigkeitsexperimenten im Labor nachzubilden versucht (z.B. Nataf et al., [1981], Davaille, [1999]). Laborexperimente spielen eine wichtige Rolle im Verständnis von Plumes. In einigen Tankexperimenten werden Plumes analog zur Erde an einer thermischen Grenzschicht am Gefäßboden (z.B. Olson et al., [1988]) erzeugt. In anderen, sogenannten Zweiflüssigkeitsexperimenten werden Plumes untersucht, die dadurch entstehen, daß man eine viskose Flüssigkeit geringer Dichte und Viskosität in eine andere Flüssigkeit höherer Dichte und Viskosität injiziert (z.B. Olson und Singer, [1985]). Olson und Christensen [1986] und Whitehead und Helfrich [1988] untersuchen in derartigen Experimenten die Ausbreitung von Volumenstörungen in Form von solitären Wellen in einem stationären Plumekanal. Solitäre Wellen sind Wellenphänomene, deren Ausbreitungsgeschwindigkeiten von ihren Amplituden abhängen und die über große Ausbreitungsentfernungen ihre Form beibehalten.

Die in diesen Zweiflüssigkeitsexperimenten beobachteten Plumes zeigen zwar in vielen Aspekten ein ähnliches Verhalten wie die thermischen, sind aber als Modelle für die Mantelplumes der Erde nur eingeschränkt geeignet. Im Gegensatz zu der in thermischen Plumes 
auftretenden thermischen Diffusion ist die chemische Diffusion bei den durch Dichtekontraste angetrieben Plumes um einige Größenordnungen kleiner und spielt eine untergeordnete Rolle. Experimente und numerische Rechnungen zeigen aber, daß die thermische Diffusion in Plumeköpfen (Griffiths, [1986]) und Plumekanälen der Erde (Albers und Christensen, [1996]) einen nicht zu vernachlässigenden Einfluß hat. Daher wird in der hier vorliegenden Arbeit die Modellierung eines rein thermisch getriebenen Plumes vorgestellt. Als Modellflüssigkeit wird ein Glukosesirup verwendet, dessen Viskosität stark von der Temperatur abhängt. Am Boden eines mit kaltem Sirup gefüllten Plexiglasgefäßes wird heißer Sirup injiziert und dadurch ein Plume erzeugt. Durch das Injizieren ist der Volumenfluß des heißen Materials genau kontrollierbar und der Aufstiegsort des Plumes fest vorgegeben, was für die experimentelle Untersuchung große Vorteile gegenüber der Plumeerzeugung an einer thermischen Grenzschicht hat. Im Gegensatz zu den genannten Zweiflüssigkeitsexperimenten tritt hier allerdings die Schwierigkeit auf, daß sich das Plumematerial nur durch seine Temperatur vom Umgebungsmaterial unterscheidet und die Strömung nicht direkt visuell zu beobachten ist, wie es bei chemischen Plumes, zum Beispiel durch Einfärben des leichteren Materials, zu erreichen wäre. Gebräuchliche Beobachtungsmethoden, wie z.B. das Schlierenverfahren (z.B. Eckelmann, [1997]) oder einfach nur die Beobachtung von Schattenbildern der mit parallelem Licht durchstrahlten Strömung (Chen und Whitehead, [1968]) (Schattenverfahren) können den Plume zwar visualisieren, aber dabei nur qualitative Aussagen über die Temperaturen liefern. Für ein genaueres Verständnis der Plumedynamik ist aber die Kenntnis des radialen und vertikalen Temperaturverlaufs im Plume von großer Bedeutung. Da dieser jedoch nicht direkt zugänglich ist, weil eingebrachte Thermometer oder Thermoelemente die sensible Strömung stark beeinflussen könnten, wird in dieser Arbeit eine berührungslose Temperaturmeßmethode angewandt (Laudenbach und Christensen, [2001]). Diese macht es sich zunutze, daß der Brechungsindex bei den für gewöhnlich verwendeten Flüssigkeiten temperaturabhängig und der aufsteigende Plume axialsymmetrisch ist. Dabei werden die horizontalen Ablenkungswinkel gemessen, die ein Laserstrahl beim Durchlaufen des Plumes in verschiedenen radialen Abständen von Plumezentrum erfährt. Das der Seismologie entlehnte Wiechert-Herglotz Verfahren (z.B. Fowler, [1990]) wird in leicht abgewandelter Form dazu verwendet, die Daten der Strahlablenkung zu invertieren. Die Lösung der Inversion liefert den radialen Verlauf des Brechungsindexes und damit auch den Temperaturverlauf in der Strömung. Der experimentelle Aufbau ist so konzipiert, daß die Messung der Ablenkungswinkel so schnell erfolgen kann, daß die Methode auch für instationäre Plumeströmungen anwendbar ist. Neben den Temperaturen sind insbesondere im Hinblick auf zeitabhängige Vorgänge auch die Strömungsgeschwindigkeiten von großem Interesse. Zu deren Untersuchung stellt die Strömungsmeßtechnik mit der PIV-Methode (Particle-Image-Velocimetry, z.B. Eckelmann, [1997]) eine etablierte und viel genutzte optische Geschwindigkeitsfeldmeßmethode zur Verfügung, die in dieser Arbeit ebenfalls Verwendung findet.

Bei der Betrachtung instationärer Vorgänge sind die, sich im Plumekanal ausbreitenden, Volumenstörungen von besonderem Interesse und stellen eine Hauptmotivation für die in dieser Arbeit beschriebenen Untersuchungen dar. Schubert et al. [1989] zeigen mit einem 
numerischen Modell, daß die von Olson und Christensen [1986] und Whitehead und Helfrich [1988] experimentell beobachteten solitären Wellen auch in rein thermischen Plumekanälen aufsteigen können. Man vermutet, daß derartige Wellen die Ursache für einen zeitlich stark variierenden Hotspot-Vulkanismus sind, da sie immer wieder große Mengen heißen Materials sehr schnell unter die Lithosphäre transportieren und auf diese Weise einen über Millionen Jahre hinweg ,pulsierenden“ Hotspot erzeugen könnten. In der hier vorliegenden Arbeit werden Volumenstörungen in Form von solitären Wellen auch im Experiment erzeugt und mit Hilfe der oben genannten Meßmethoden untersucht.

Zusätzlich zu der experimentellen Modellierung wird in dieser Arbeit auch eine numerische Modellierung thermischer Plumes vorgestellt. Diese Umsetzung orientiert sich zunächst sehr stark an dem Experiment, um die direkte Vergleichbarkeit zwischen beiden Modellierungen zu gewährleisten, so daß eine bessere Beurteilung der Ergebnisse möglich wird. Insbesondere die Qualität der experimentellen Temperaturmeßmethode läßt sich damit leichter abschätzen.

Mit Hilfe der numerischen Modellierung werden systematische Parameterstudien im Hinblick auf die Wellenausbreitung in thermischen Plumes durchgeführt, die es ermöglichen, die experimentellen Resultate in einen allgemein gültigen Kontext einzuordnen. Dabei sind auch Parameterbereiche untersucht worden, die experimentell nur schwer oder im Fall einer tiefen- bzw. druckabhängigen Viskosität überhaupt nicht zugänglich sind.

Die in den Parameterstudien erzielten Ergebnisse werden auf die Erde skaliert und ihre Bedeutung für verschiedene Mantelplumes der Erde diskutiert. Dabei wird insbesondere untersucht, ob die durch die Wellen transportierten Temperaturanomalien einen Einfluß auf die Aktivität von Hotspots haben können.

Zusammenfassend läßt sich feststellen, daß das in dieser Arbeit vorgestellte Experiment die Effekte der thermischen Diffusion auf den Plumekanal und auf solitäre Wellen berücksichtigt und gegenüber anderen experimentellen Plumemodellierungen (z.B. Griffiths und Campbell, [1990]) den Vorteil hat, daß es die störungsfreie Bestimmung der für die Dynamik von thermischen Plumes wichtigen physikalischen Größen ermöglicht. Deshalb können hier erstmals direkte quantitative Vergleiche mit einer numerischen Modellierung eines thermischen Plumes angestellt werden. Das hat zum einen den Vorteil, daß im Falle einer Übereinstimmung zwischen den Modellierungen, die Numerik aufwendige experimentelle Parameterstudien ersetzen kann und zum anderen die Ergebnisse von Rechnungen mit erdähnlicheren Parametern in einem gesicherten Zusammenhang erscheinen. Das für die Vergleiche entwickelte zweidimensionale Konvektionsprogramm „CFDC“ hat im Gegensatz zu dreidimensionalen Programmen den Vorteil, daß es bei den für die Untersuchung von solitären Wellen erforderlichen langgestreckten Untersuchungsgebieten mit einem vertretbaren Speicher- bzw. Rechenzeitaufwand arbeitet, so daß umfangreiche Parameterstudien überhaupt erst möglich werden.

Die Arbeit gliedert sich so, daß im nachfolgenden zweiten Kapitel zunächst der experimentelle Versuchsaufbau, die verwendeten Meßmethoden sowie einige Beispielresultate dargestellt werden. Im Kapitel 3 wird auf die numerische Modellierung des Experiments 
eingegangen und im vierten Kapitel werden die experimentellen Daten mit den numerischen Resultaten verglichen und diskutiert. Dabei werden an einigen Beispielen auch bestimmte Plume- und Welleneigenschaften verdeutlicht und diskutiert. Anschließend folgen im fünften Kapitel systematische Studien zur Wellenausbreitung in thermischen Plumes. Durch Skalierungsgesetze werden aus den numerischen Resultaten allgemeingültige Beschreibungen des Wellenausbreitungsverhalten abgeleitet. Außerdem wird die durch die Wellen erzeugte Temperaturanomalie quantifiziert und der Einfluß einer tiefenabhängigen Viskosität diskutiert. Am Ende des fünften Kapitels werden die Ergebnisse der Parameterstudien auf die Erde skaliert. In der Diskussion im sechsten Kapitel werden diese Ergebnisse angewandt um geophysikalische Aussagen über den Einfluß von solitären Wellen auf den Hotspot-Vulkanismus abzuleiten. Am Ende der Arbeit folgt der Diskussion eine Zusammenfassung und ein Anhang. In diesem sind eine Aufstellung der Sirupeigenschaften, einige Formeln sowie verwendete Skalierungen und eine Auflistung der verwendeten Geräte zu finden. 


\section{Kapitel 2}

\section{Experiment und Meßmethoden}

In diesem Kapitel werden der experimentelle Aufbau und die verwendeten Meßmethoden beschrieben. Da es sich bei dem Experiment um eine Weiterentwicklung des in Laudenbach [1997] beschrieben Aufbaus handelt, wird hier nur auf die wichtigsten Punkte und die angebrachten Veränderungen eingegangen. Eine Darstellung der Versuchsdetails, der Versuchsvorbereitung und der Durchführung finden sich in der oben genannten Arbeit.

\subsection{Aufbau des Plumeexperiments}

Im Experiment wird ein Glukosesirup (Anhang A.1) verwendet, der sich wegen der exponentiellen Abhängigkeit der Viskosität $\eta$ von der Temperatur $T$ sehr gut als Modellsubstanz für den Erdmantel eignet. Für die Abhängigkeit der Viskosität von der Temperatur wird ein modifiziertes Arrheniusgesetz angesetzt:

$$
\eta_{S}(T)=\eta_{S o} \exp \left(\frac{1}{a+b T}\right)
$$

Dabei sind $a, b$ und $\eta_{S o}$ Parameter, die durch Viskositätsmessungen bei verschiedenen Temperaturen $T$ in ${ }^{\circ} \mathrm{C}$ bestimmt sind. Typische Werte für diese Parameter sind $\eta_{S o}=$ $3.35 \times 10^{-5}$ Pas, $a=5.2 \times 10^{-2}, b=7 \times 10^{-4} K^{-1}$. Für den experimentell relevanten Bereich zwischen $10^{\circ} \mathrm{C}$ und $100^{\circ} \mathrm{C}$ variiert damit die Viskosität über vier Größenordnungen (Anhang A.1.1).

Der experimentelle Aufbau (Abbildung 2.1) besteht aus einem rechteckigen Plexiglasgefäß mit einer Grundfläche von $20 \mathrm{~cm} \times 20 \mathrm{~cm}$ und einer Höhe von $150 \mathrm{~cm}$, das mit Sirup der Raumtemperatur $T_{o}$ gefüllt ist. Der heiße Sirup wird in einem Metalldruckbehälter in einem Wasserbad auf konstanter Temperatur gehalten und durch die $7 \mathrm{~mm}$ weite Öffnung in das Plexiglasgefäß injiziert. Mit Hilfe eines computergesteuerten Schrittmotors, der den Kolbenvorschub einer Spritze kontrolliert, läßt sich der Volumenfluß $Q=d V / d t$ des injizierten Materials sehr genau regeln. Das durch den Kolben verdrängte Silikonöl drückt die gleiche Menge heißen Materials durch ein Steigrohr im Druckbehälter in das Plexiglasgefäß. 


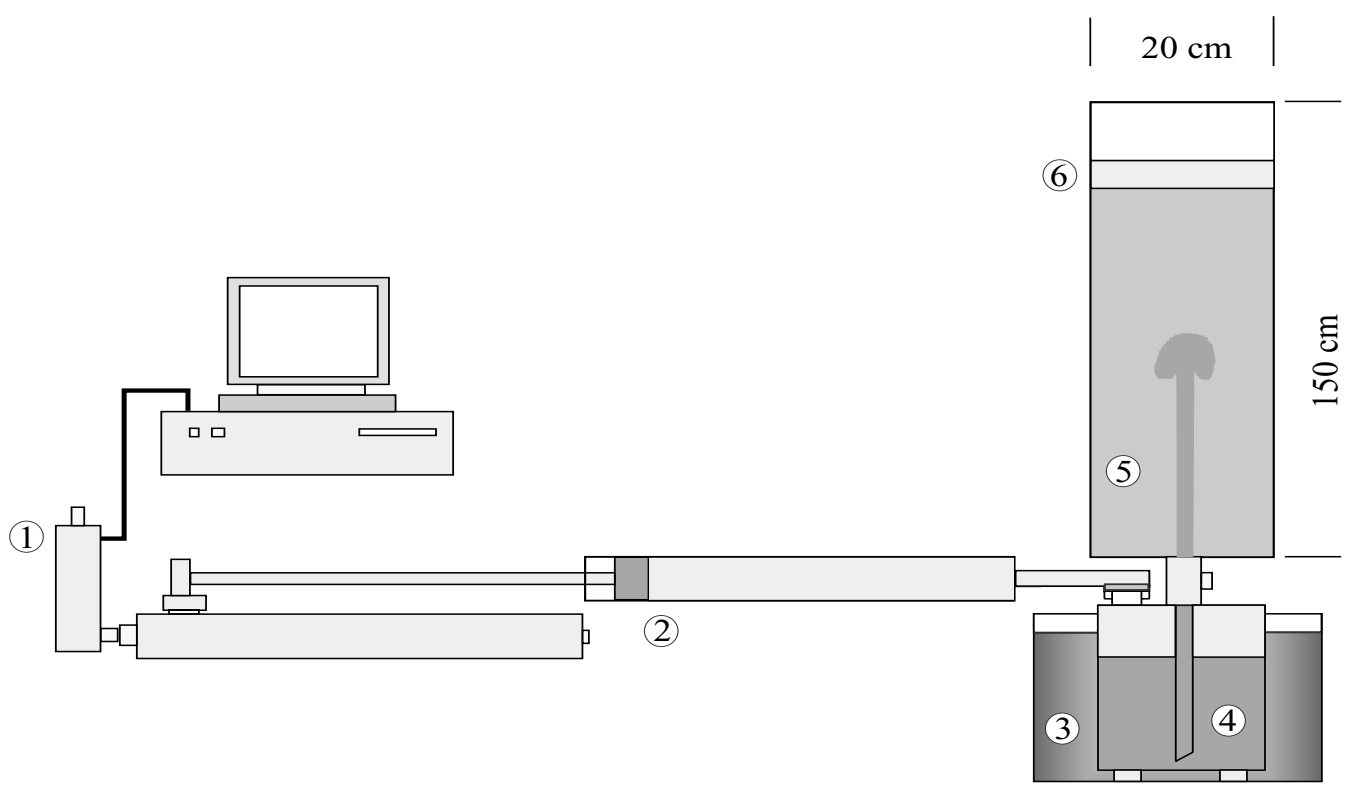

Abbildung 2.1: Versuchsaufbau: 1. Computergesteuerter Schrittmotor; 2. Kolbenspritze gefüllt mit Silikonöl; 3. Wasserbad; 4. Druckbehälter mit heißem Sirup und Steigrohr; 5. Plexiglasgefäß gefüllt mit Sirup; 6. Dünne Ölschicht

Die Temperatur des einströmenden Sirups am Gefäßboden läßt sich durch ein Thermoelement in der Eintrittsöffnung und die Matrixtemperatur durch ein Quecksilberthermometer an der Gefäßwandung bestimmen. Um einen Wasserverlust des Sirups durch Verdunstung zu verhindern, ist auf die Sirupoberfläche eine dünne Ölschicht aufgebracht. Zu Beginn eines Experiments wird ein konstanter Volumenstrom heißen Materials injiziert. Dabei bildet sich zunächst ein pilzkopfartiger Diapir (Abbildung 2.2 a), dem ein Plumekanal nachfolgt. Der Diapir vergrößert sich während des Aufstiegs durch spiralartiges Einbinden von Umgebungsmaterial (engl.: entrainement). Besitzt er eine ausreichend große Temperaturanomalie, so erreicht er die Flüssigkeitsoberfläche, wo er sich in Form einer warmen Schicht ausbreitet und sich dabei langsam (thermisch) auflöst. Der übrigbleibende stationäre Kanal (Abbildung 2.2 b) stellt die Voraussetzung zur Erzeugung solitärer Wellen dar. Sie werden im Experiment durch kurzzeitiges Erhöhen des vorgegebenen Volumenstroms erzeugt.

In Abbildung 2.3 ist eine derartig erzeugte Materialwelle dargestellt. Im Moment des vergrößerten Volumenflusses wurde etwas grüne Farbe zusätzlich in den Kanal injiziert. Man erkennt, daß die Farbe die Welle nicht verläßt. In einem sich mit der Welle nach oben bewegenden Referenzkoordinatensystem zeichnet die Farbe die geschlossenen Stromlinien der Welle nach. Whitehead und Helfrich [1988] machen in ihren Zweiflüssigkeitsexperimenten ähnliche Beobachtungen.

Zur Temperaturbestimmung in stationären Kanälen und solitären Wellen wird das nachfolgende Meßverfahren verwendet. Dieses weicht zwar nur in einigen wenigen Punkten von dem in Laudenbach [1997] dargestellten Verfahren ab, soll aber wegen seiner großen Bedeutung für diese Arbeit dennoch hier beschrieben werden. 
a)

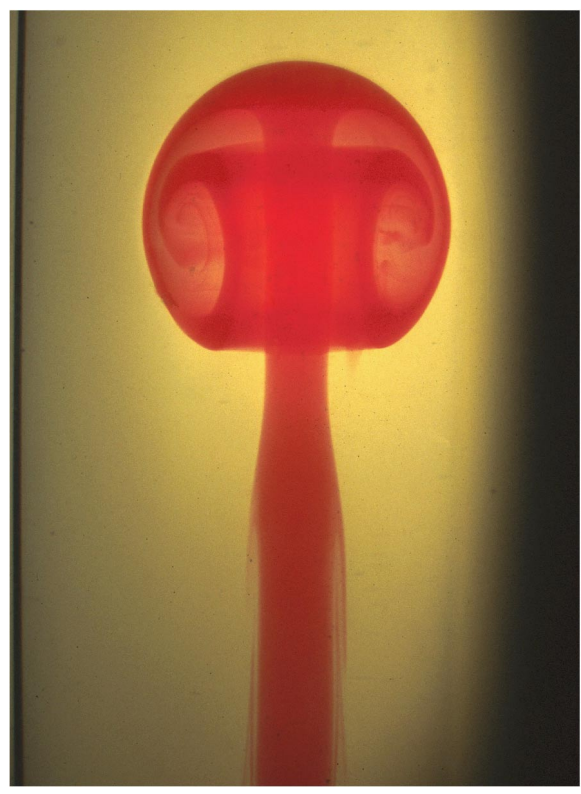

b)

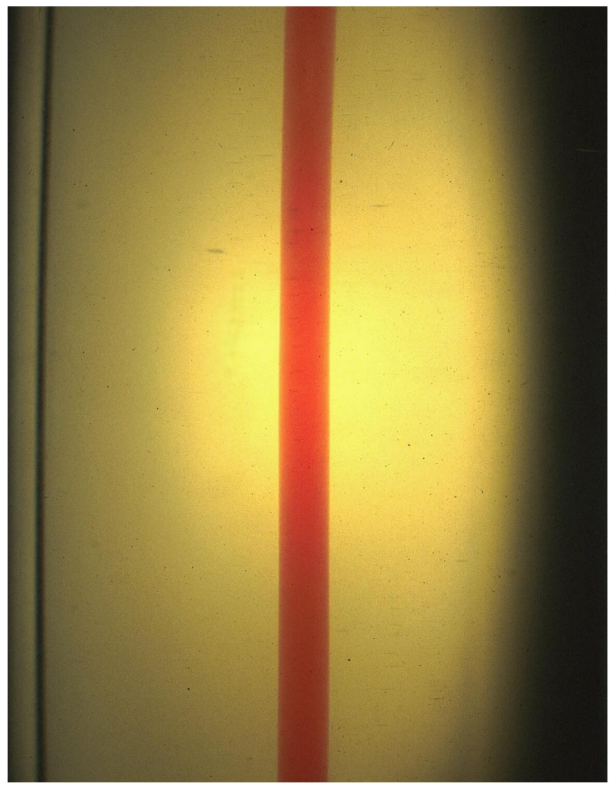

Abbildung 2.2: Fotografie eines Diapirs (a) und eines stationären Kanals (b). Zur Visualisierung wurde das injizierte Material rot eingefärbt. (Bildmaßstab ca. 1:3)

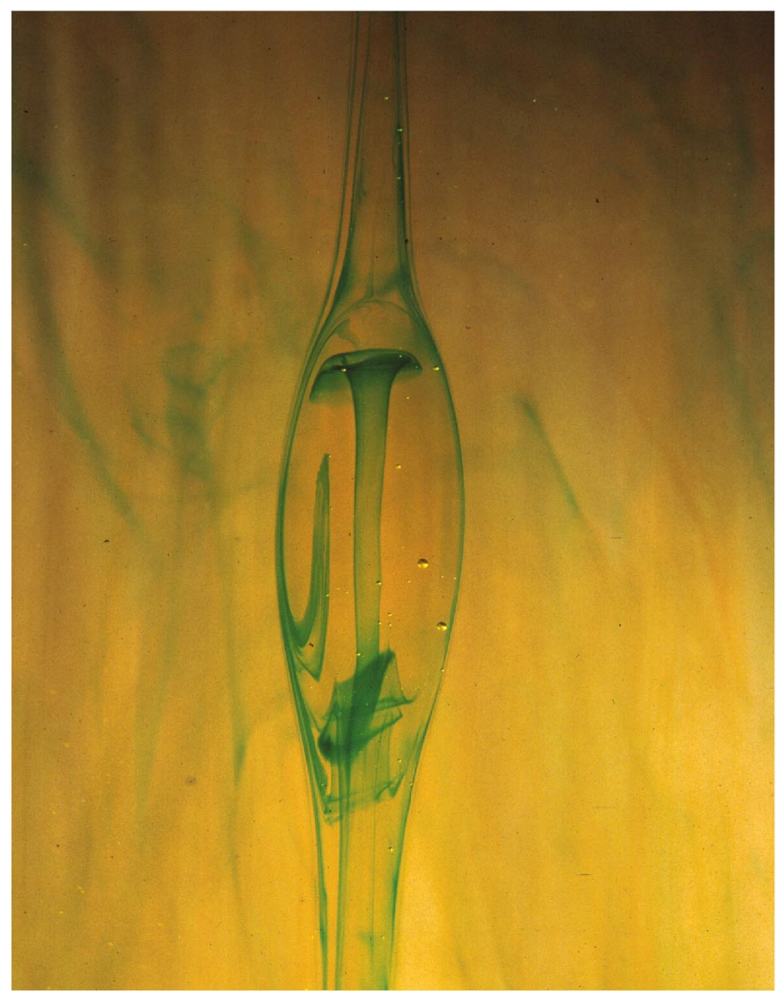

Abbildung 2.3: Fotografische Aufnahme einer solitären Welle. Im Moment des erhöhten Volumenflusses wurde etwas Farbe injiziert, die die geschlossenen Stromlinien in einem sich mitbewegenden Referenzkoordinatensystem nachzeichnet. Die Farbe verläßt die Welle nicht. (Bildmaßstab ca. 1:2.5) 


\subsection{Temperaturmeßmethode}

In den folgenden Abschnitten werden die theoretischen Grundlagen der Temperaturmeßmethode, der praktische Aufbau und einige Beispielresultate dargestellt. Wesentliche Teile davon sind Laudenbach [2001] entnommen. Die Methode basiert ursprünglich auf einem Experiment von Herbert und LeContel [1991], die aus den Ablenkungswinkeln eines mit Laserstrahlen durchstrahlten Laborplumes die radialen Temperaturen in quasi-stationären Plumeströmungen bestimmen. Die in der hier vorliegenden Arbeit beschriebene Methode ist aber insbesondere auch im Hinblick auf die Untersuchung von solitären Wellen, also auch für stark zeitabhängige, instationäre Vorgänge konzipiert worden.

\subsubsection{Theorie}

Mit zunehmender Temperatur nimmt der Brechungsindex des Sirups nahezu linear ab (Abbildung A.1.2). Dadurch wirkt ein heißer Aufstrom für einfallende Strahlen in horizontaler Richtung wie eine Zerstreuungslinse mit einer sehr schwachen Brechkraft. Es wird angenommen, daß der Plume axialsymmetrisch ist und sich seine Eigenschaften nur geringfügig mit der Höhe verändern, so daß er in einer konstanten Aufstiegshöhe näherungsweise als Zylinder behandelt werden kann und sich die physikalischen Größen nur mit dem Radius $r$ ändern. Zur Bestimmung radialer Temperaturprofile werden die horizontalen Ablenkungswinkel von Laserstrahlen gemessen, die in unterschiedlichen Entfernungen zur Plumeachse die Strömung passieren. Der Strahlverlauf wird durch das Snelliussche Brechungsgesetz für kontinuierliche Medien beschrieben. Da die Symmetrie in der Ausbreitungsebene des Strahls die gleiche ist wie in einem eindimensionalen Kugelmodel, können hier Analogien zur Strahlenseismologie in einer radialsymmetrischen Erde genutzt werden. Analog zum seismischen Strahlparameter, kann auch hier jedem Laserstrahl ein Strahlparameter zugeordnet werden:

$$
p=n(r) r \sin (i)
$$

Dabei ist $r$ der Radius vom Plumezentrum aus betrachtet, $n(r)$ der Brechungsindex, der umgekehrt proportional zur Lichtgeschwindigkeit in der Flüssigkeit ist und $i$ der Winkel zwischen dem Radiusvektor und der Strahltangente an einem beliebigen Punkt (vergl. Abbildung 2.4).

Der Strahlparameter $p$ ist entlang eines einzelnen Strahls konstant. Er steht über $p=n_{o} x$ mit der Distanz $x$, der Entfernung zwischen dem einfallenden Strahl und der Plumeachse in Beziehung, wobei $n_{0}$ der Brechungsindex fern ab vom Plumezentrum ist.

Für den, der seismischen Epizentralentfernung analogen, Winkel $\Delta$ findet sich folgender Ausdruck (z.B. Lay und Wallace, [1995])

$$
\Delta(p)=2 p \int_{r_{o}}^{R} \frac{d r}{r\left(\eta^{2}-p^{2}\right)^{\frac{1}{2}}},
$$




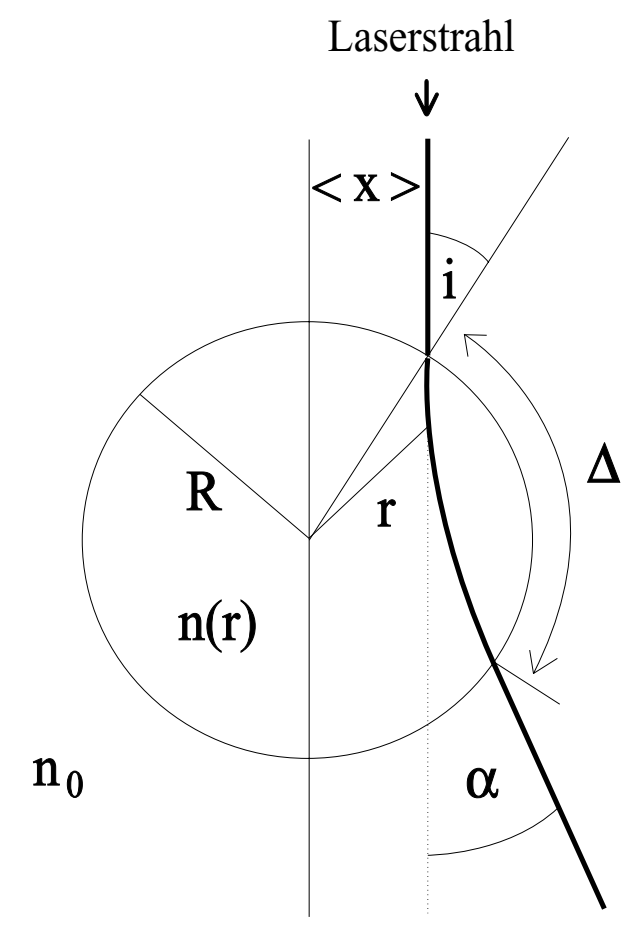

Abbildung 2.4: Schematische Darstellung der Laserstrahlablenkung im Plume. Der Kreis mit dem Radius $R$ kennzeichnet die thermisch inhomogene Region der Strömung, in der der Brechungsindex $n$ mit dem Radius $r$ monoton zunimmt. Für $r>R$ nimmt der Brechungsindex den konstanten Wert $n_{o}$ an.

wobei $\eta$ durch $\eta=n(r) r$ definiert ist und $r_{o}$ die kürzeste Entfernung zum Plumezentrum darstellt. Für $R \rightarrow \infty$ ergibt sich für den Ablenkungswinkel $\alpha=\pi-\Delta$.

Unter der berechtigten Annahme, daß $\eta$ mit zunehmendem $r$ monoton anwächst, kann man die Integrationsvariable $r$ in Gleichung 2.3 durch $\eta$ substituieren. Damit läßt sich

$$
\alpha(p)=2 p \int_{p}^{\infty} \frac{1}{n\left(\eta^{2}-p^{2}\right)^{\frac{1}{2}}} \frac{d n}{d \eta} d \eta
$$

als Ausdruck für den Ablenkungswinkel herleiten. Gleichung 2.4 stellt die Lösung für das Vorwärtsproblem dar. D.h., daß man damit aus einer gegebenen Temperaturverteilung die zu erwartenden Laserstrahlablenkungswinkel in Abhängigkeit von der Entfernung $x$ bestimmen kann. Für den in Abbildung 2.5 a) dargestellten hypothetisch angenommenen Gaußschen Temperaturverlauf im Plume mit einer Amplitude von $75^{\circ} \mathrm{C}$ und einer Halbwertsbreite von $18 \mathrm{~mm}$ läßt sich mit Gleichung 2.4 und der Temperaturabhängigkeit des Brechungsindexes (A.1.2) die in Abbildung 2.5 b) gezeigte theoretische Laserstrahlablenkungskurve bestimmen. Der maximal zu erwartende Ablenkungswinkel liegt bei ungefähr $1.5^{\circ}$, was hohe Anforderungen an die Genauigkeit des experimentellen Aufbaus stellt.

Um aus gemessenen Ablenkungswinkeln radiale Temperaturverteilungen bestimmen zu können, muß das Umkehrproblem gelöst werden. Das geschieht analog zu der in der Seis- 
a)
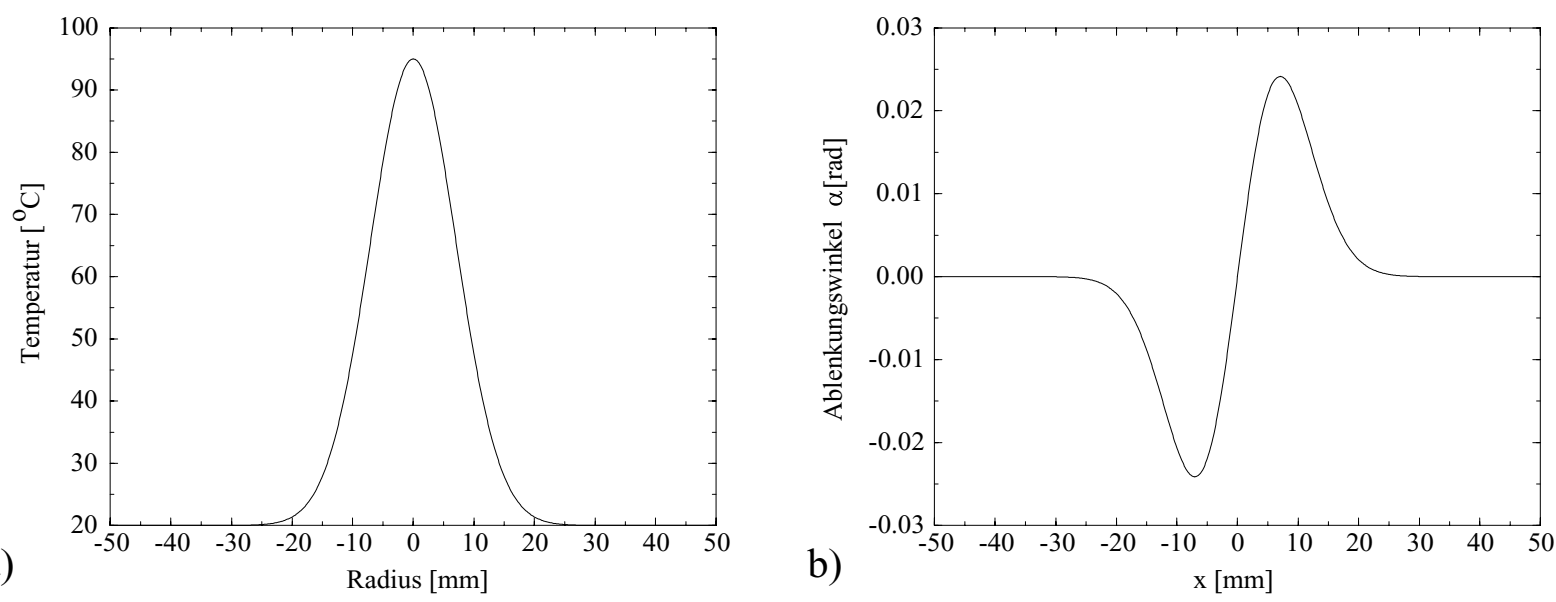

Abbildung 2.5: Hypothetisch angenommener Temperaturverlauf (a) und daraus bestimmte synthetische Ablenkungskurve (b).

mologie verwendeten Wiechert-Herglotz-Laufzeitinversion für seismische Wellen (Lay und Wallace, [1995]). Das Ergebnis lautet:

$$
\int_{x}^{\infty} \frac{\alpha\left(x^{\prime}\right)}{\left(x^{\prime 2}-x^{2}\right)^{\frac{1}{2}}} d x^{\prime}=\pi \ln \left[\frac{n_{o}}{n\left(r_{o}\right)}\right]
$$

Dabei ist die Integrationsvariable $p$ durch $x=p / n_{o}$ ersetzt worden. Um die Singularität bei $x^{\prime}=x$ zu umgehen, wird eine partielle Integration durchgeführt woraus sich

$$
n\left(r_{o}\right)=n_{o} \exp \left(\frac{1}{\pi} \int_{x}^{\infty}\left[1-\left(\frac{x}{x^{\prime}}\right)^{2}\right]^{\frac{1}{2}}\left\{\frac{d \alpha}{d x^{\prime}}-\frac{\alpha\left(x^{\prime}\right)}{x^{\prime}}\right\} d x^{\prime}\right)
$$

ergibt. Da im Experiment der Ablenkungswinkel eines Strahls schon mit endlicher Entfernung $x_{o}$ vom Plumezentrum Null wird, kann man $\infty$ in der oberen Integrationsgrenze durch $x_{o}$ ersetzen. Des weiteren steht $r_{o}$ zu $x$ über den Faktor $n_{o} / n$ in Beziehung, der für einen Temperaturkontrast von bis zu $70^{\circ} \mathrm{C}$ um weniger als ein Prozent von eins abweicht, so daß sich $r_{o}$ auf der linken Seite von Gleichung 2.6 durch $x$ ersetzen läßt. Mit Hilfe der empirisch bestimmten Temperaturabhängigkeit des Brechungsindexes (A.1.2) wird schließlich der gewünschte radiale Temperaturverlauf $T(r)$ bestimmt. Insgesamt benötigt man für ein vollständiges Temperaturprofil zusätzlich zu den Ablenkungswinkeln der Strahlen zwischen 0 und $x_{o}$ nur eine unabhängig zu messende Temperatur, nämlich die des Materials fern ab vom Plume, zur Bestimmung von $n_{0}$.

\subsubsection{Aufbau}

Der experimentelle Aufbau der Temperaturmeßmethode (Abbildung 2.6) ermöglicht es, in jeder Sekunde die Ablenkungswinkel für ein Temperaturprofil aufzuzeichnen. Dazu wird 


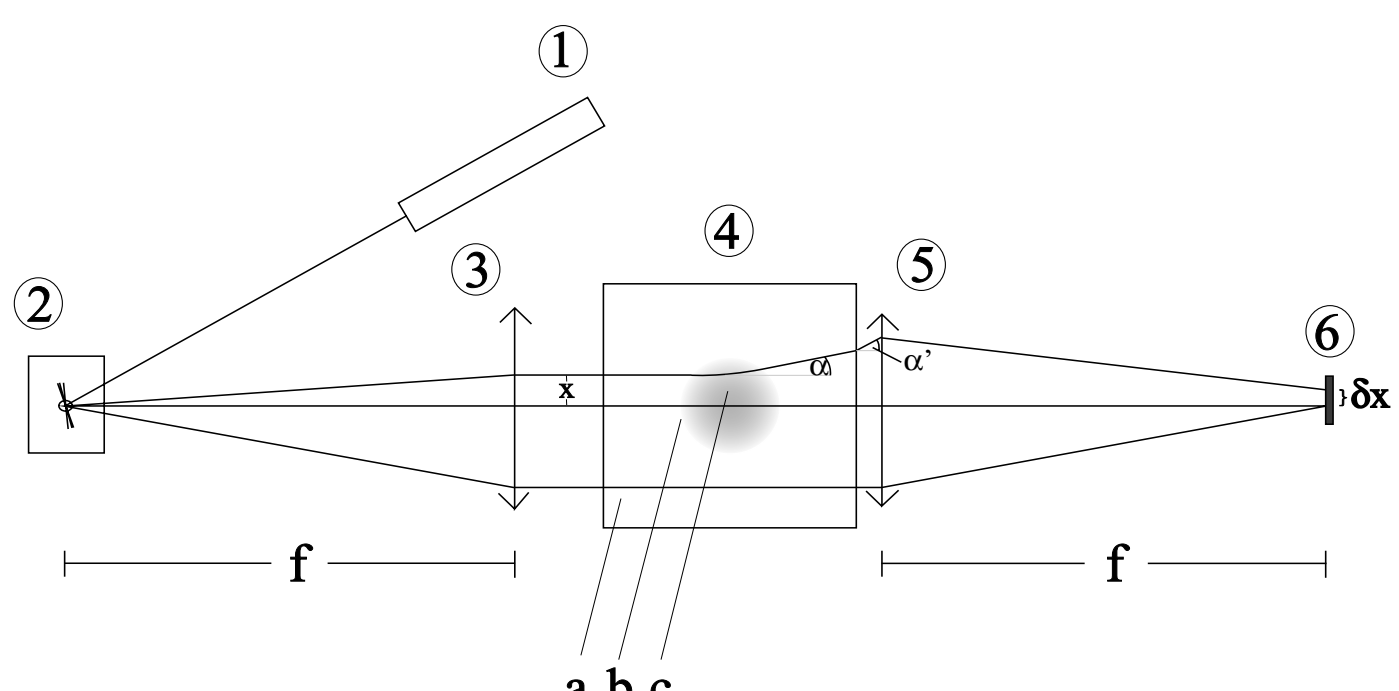

Abbildung 2.6: Aufbau der Temperaturmeßmethode (von oben betrachtet). 1. Laser, 2. Galvanoscanner mit Spiegel, 3. Linsensystem mit Brennweite f, 4. Plexiglastank, 5. Linsensystem, 6. CCD - Zeilenkamera mit Polarisationsfiltern. Es sind exemplarisch drei verschiedene Strahlverläufe (a, b, c) eingezeichnet.

der Strahl eines HeNe-Lasers (1) von dem oszillierenden Spiegel eines Galvanoscanners (2) in unterschiedlichen Winkeln zum Tank hin reflektiert. Ein Linsensystem (3) mit $10 \mathrm{~cm}$ Durchmesser und einer Brennweite von $f=42 \mathrm{~cm}$, dessen Brennpunkt auf dem Schwingspiegel zu liegen kommt, stellt sicher, daß alle Strahlen senkrecht in das Gefäß (4) einfallen. Die Ansteuerung des Galvanoscanners erfolgt mit einer Dreiecksspannung, so daß der Laserstrahl das Gefäß mit konstanter Geschwindigkeit zwischen zwei Umkehrpunkten überstreicht. Hinter dem Gefäß befindet sich ein zweites Linsensystem (5) mit einem Durchmesser von $10 \mathrm{~cm}$ und einer Brennweite von $f=42 \mathrm{~cm}$, dessen Brennpunkt im Zentrum einer CCD-Zeilenkamera (6) liegt. Dadurch werden Strahlen, die ausschließlich durch homogenen Sirup bzw. genau durch das Plumezentrum verlaufen nicht abgelenkt und im Zentrum der CCD-Kamera detektiert (Strahlen a,b in Abbildung 2.6).

Alle anderen Strahlen werden durch die Linsenwirkung des heißen Plumes vom Zentrum unter dem Winkel $\alpha$ weg gebrochen (z.B. Strahl c, Abbildung 2.6). Beim Austritt aus dem Gefäß wird der Strahl ein zweites Mal um den Winkel $\alpha^{\prime}$ gebrochen. Für kleine Winkel von $\alpha$ gilt $\alpha^{\prime}=n_{o} \alpha$. Strahlen, die das Gefäß unter dem Winkel $\alpha^{\prime}$ verlassen, treffen auf der CCD-Zeilenkamera mit einer Wegdifferenz

$$
\delta x=f \alpha^{\prime}=f n_{o} \alpha
$$

zum Zentrum auf. Aus dem Schwerpunkt der von der CCD-Kamera gemessenen Intensitätsverteilung wird jeweils die Wegstrecke $\delta x$ bestimmt (vergl. Abbildung 2.6). Die Kamera arbeitet während einer Aufnahmesequenz mit einer konstanten Aufnahmefrequenz von 470 Hz. Während einer Sequenz werden die gemessenen Intensitätsverteilungen zunächst im 
Arbeitsspeicher des Steuerrechners abgelegt und anschließend für die weitere Auswertung durchnumeriert und auf der Festplatte gesichert. Der Laserstrahleintrittsort $x$ in das Gefäß ist eine lineare Funktion der verstrichenen Zeit seit Beginn der Aufnahmesequenz und wird daher aus den Nummern der abgespeicherten Intensitätsverteilungen bestimmt.

Um Messungen in unterschiedlichen Aufstiegshöhen des Plumes durchführen zu können, ist der optische Aufbau auf einer um das Experiment herumgreifenden Platte montiert, deren Lage in der Höhe verändert werden kann. Aus konstruktionstechnischen Gründen liegt die tiefstmögliche Meßhöhe etwa $15 \mathrm{~cm}$ über der Einströmöffnung, was aber kein Problem darstellt, da die Einströmtemperatur durch das Thermoelement am Einströmpunkt bekannt ist und in derartig niedrigen Aufstiegshöhen nicht mit einer starken Auskühlung des Plumes zu rechnen ist.

Die wesentlichste Änderung, die im Vergleich zu dem in Laudenbach [1997] beschrieben Aufbau vorgenommen wurde, besteht in der Verwendung von abbildungskorrigierten Linsensystemen anstelle von sphärischen Einzellinsen. Besonders für Strahlen fern ab der optischen Achse zeigen Einzellinsen eine starke sphärische Aberration, die schon bei homogenem Sirup deutliche Wegdifferenzen $\delta x$ der einfallenden Laserstrahlen auf der Kamera verursacht. Abbildung 2.7 zeigt exemplarische Laserstrahlablenkungskurven, gemessen bei einem Plumekanal in niedriger Aufstiegshöhe für eine sphärische Einzellinse (a) und für ein korrigiertes Linsensystem (b). Die als Fehlerkurven bezeichneten Graphen stellen jeweils die bei homogenem Sirup gemessenen Ablenkungsdaten dar. Sie zeigen deutlich den Unterschied der verwendeten Linsen. Während die Einzellinse den charakteristischen Verlauf der Ablenkungskurve so stark beeinflußt, daß diese in ihren Ausläufern nicht stark genug zu Null hin abfällt (vergl. Abbildung 2.5 b), ist die Fehlerkurve des Linsensystems um eine Größenordnung kleiner und hat nahezu keinen Einfluß auf die Ablenkungskurve. Ein Herausrechnen
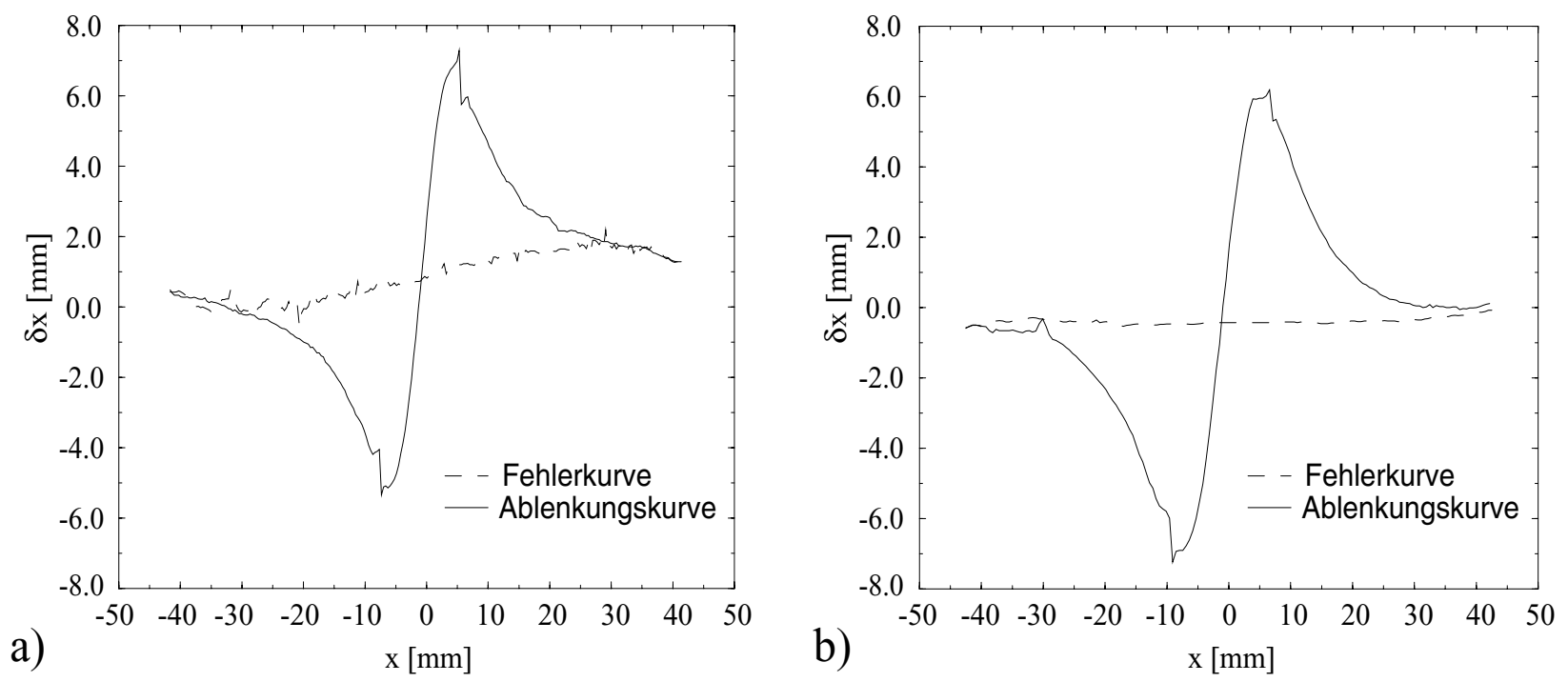

Abbildung 2.7: Exemplarische Ablenkungskurve und dazugehörige Fehlerkurve für sphärische Einzellinse (a) und für abbildungskorrigiertes Linsensystem (b). 
der Linsenfehler in den Ablenkungskurven, wie es in Laudenbach [1997] durchgeführt worden ist, ist hier nicht notwendig. Da auf der Eintrittsseite des Laserstrahls in das Gefäß die Einzellinse ebenfalls durch ein Linsensystem ersetzt worden ist, sind auch Ungenauigkeiten im parallelen Strahlverlauf minimiert worden, so daß die Daten insgesamt eine wesentlich höhere Qualität aufweisen.

\subsubsection{Auswertung und Beispielergebnisse}

Zur Auswertung werden die Ablenkungskurven zunächst in $\mathrm{x}$ - und $\mathrm{y}$-Richtung verschoben, bis ihr Symmetriepunkt mit dem Ursprung des Koordinatensystems zusammenfällt. Die Verschiebung ist nötig, weil das Plumezentrum nie exakt mit der optischen Achse des Aufbaus zusammenfällt und die Lage des Nullpunkts von $x$ und $\delta x$ über die optische Achse definiert ist. Dann werden die Absolutwerte des positiven und des negativen Astes einer jeden Kurve gemittelt. In Einzelfällen, in denen die Ablenkung für große Werte von $x$ nicht vollständig zu Null geworden ist, wird der abfallende Ast analytisch so weit ergänzt, bis sich die Kurve asymptotisch an die x-Achse angenähert hat. Nachdem die Wegstrecken $\delta x(x)$ mit Gleichung 2.7 in Ablenkungswinkel $\alpha(x)$ umgerechnet worden sind, kann die Inversion durch numerische Integration von Gleichung 2.6 für jeden Wert von $r_{o}$ bzw. $x$ durchgeführt werden, so daß man den Brechungsindex als Funktion von $r_{o}$ bzw. $x$ erhält, woraus sich nach dem Zusammenhang A.1.2 der radiale Temperaturverlauf $T(r)$ ergibt.

Abbildung 2.8 a) zeigt die Ablenkungskurven für einen Plumekanal in $150 \mathrm{~mm}$ und
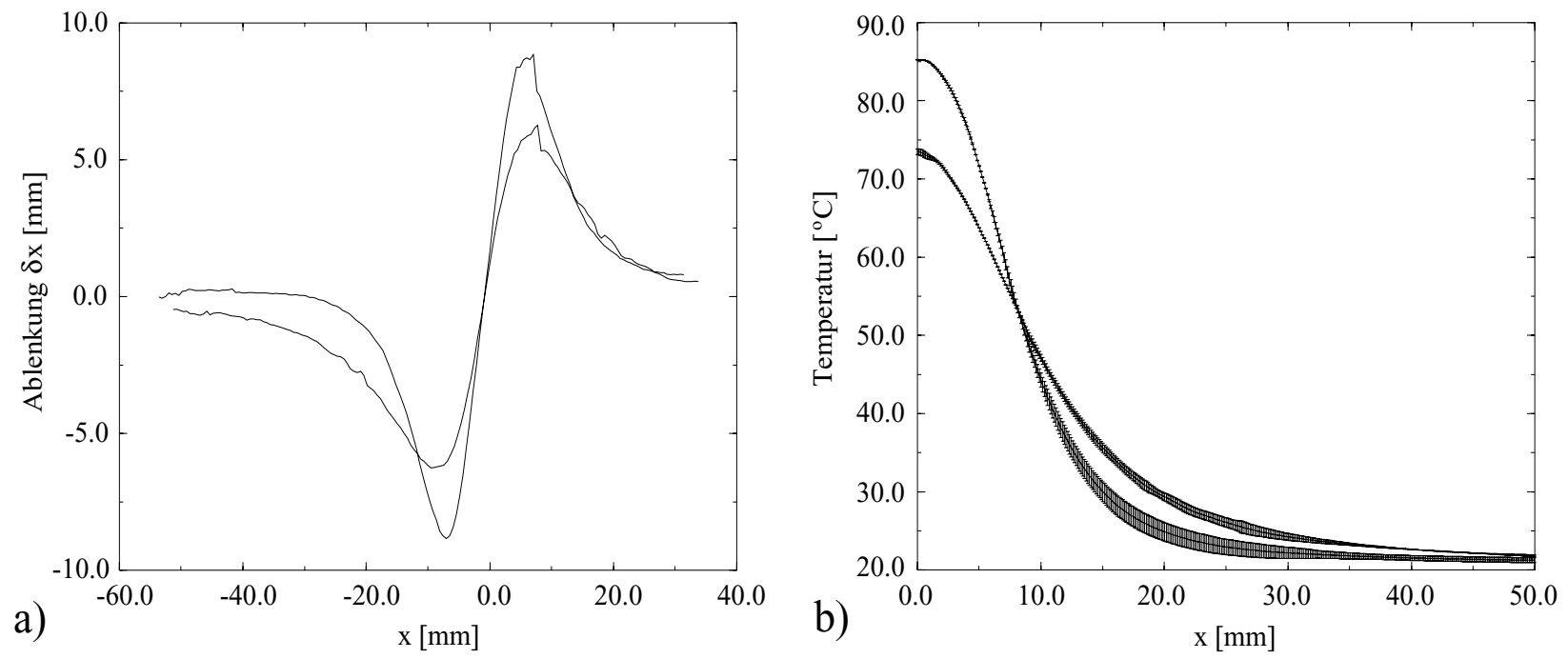

Abbildung 2.8: (a) Ablenkungskurven für einen stationären Kanal gemessen in $150 \mathrm{~mm}$ und $450 \mathrm{~mm}$ Höhe. Die Amplitude der Kurve in $450 \mathrm{~mm}$ Höhe ist kleiner als die der Kurve bei 150 mm. (b) Aus den Ablenkungskurven berechnete Temperaturprofile. Die Schattierung der Graphen markiert die Abweichung zwischen der Inversion des negativen Asts und der des positiven Asts der Ablenkungskurve. 
450 mm Höhe. Da der Plume auf seinem Weg nach oben auskühlt und sich verbreitert, nehmen die horizontalen Gradienten des Brechungsindexes ab, so daß die Amplituden der Ablenkungskurven kleiner werden. In Abbildung 2.8 b) sind die aus den Ablenkungskurven bestimmten Temperaturprofile dargestellt. Die gemessenen Ablenkungskurven stimmen prinzipiell in ihrer Form mit dem erwarteten Verlauf (vergl. synthetische Daten, Abbildung 2.5) überein, zeigen jedoch bei etwa $x=+7 \mathrm{~mm}$ kleine Sprünge, die vermutlich auf einen geringfügigen optischen Fehler einer der im Strahlengang plazierten Komponenten zurückzuführen sind. Außerdem beobachtet man eine leichte Asymmetrie der Kurven. Die Ursachen dafür könnten in einem kleinen horizontalen Temperaturgradienten in der Matrix oder einer geringen Abweichung der Plumestruktur von der Axialsymmetrie liegen. Um den Einfluß dieser Beobachtungen auf die Ergebnisse zu untersuchen, sind bei den dargestellten Ablenkungskurven die positiven und negativen Äste jeweils getrennt voneinander invertiert worden. Die sich dabei ergebenen Abweichungen sind als Schattierungen in die resultierenden Temperaturprofile (Abbildung 2.8 b) eingezeichnet worden. Es zeigt sich, daß die kleinen Sprünge nur einen geringen Einfluß von etwa 3\% auf die gemessene Plumezentrumstemperatur haben. Die leichte Asymmetrie der Kurven verursacht eine maximal 10\%-ige Unsicherheit in den Plumeausläufern. Aufgrund dieser Überlegungen wird davon ausgegangen, daß alle nachfolgend dargestellten Temperaturmeßergebnisse mit diesen Unsicherheiten behaftet sein können.

Abschließend für dieses Kapitel zeigt Abbildung 2.9 ein Beispiel für zwei vollständige ausgemessene Plumekanäle mit jeweils der gleichen Anfangstemperaturanomalie, aber mit unterschiedlichen Volumenflüssen. Für die Darstellung sind ab einer Aufstiegshöhe von $15 \mathrm{~cm}$ jeweils alle $5 \mathrm{~cm}$ Temperaturprofile aufgenommen worden. Diese sind anschließend in Form einer Konturdarstellung zusammenmontiert worden. Die kontinuierliche Darstellung ergibt sich aus der Interpolation des verwendeten Graphikprogramms. Der Plume mit einem Volumenfluß von $20 \mathrm{ml} / \mathrm{min}$ hat in der größten Aufstiegshöhe bereits so viel von seiner Anfangstemperaturanomalie verloren, daß Messungen in größeren Höhen durch den nicht vollständig aufgestiegenen Plumekopf verfälscht worden wären und daher nicht mehr durchgeführt worden sind. Der stärkere Plume mit $40 \mathrm{ml} / \mathrm{min}$ Volumenfluß kühlt auf seinem Weg nach oben weniger aus und verbreitert sich nicht so stark wie der schwache.

Weitere Ergebnisse von Temperaturmessungen und Vergleiche mit numerischen Resultaten finden sich in Kapitel 4. 


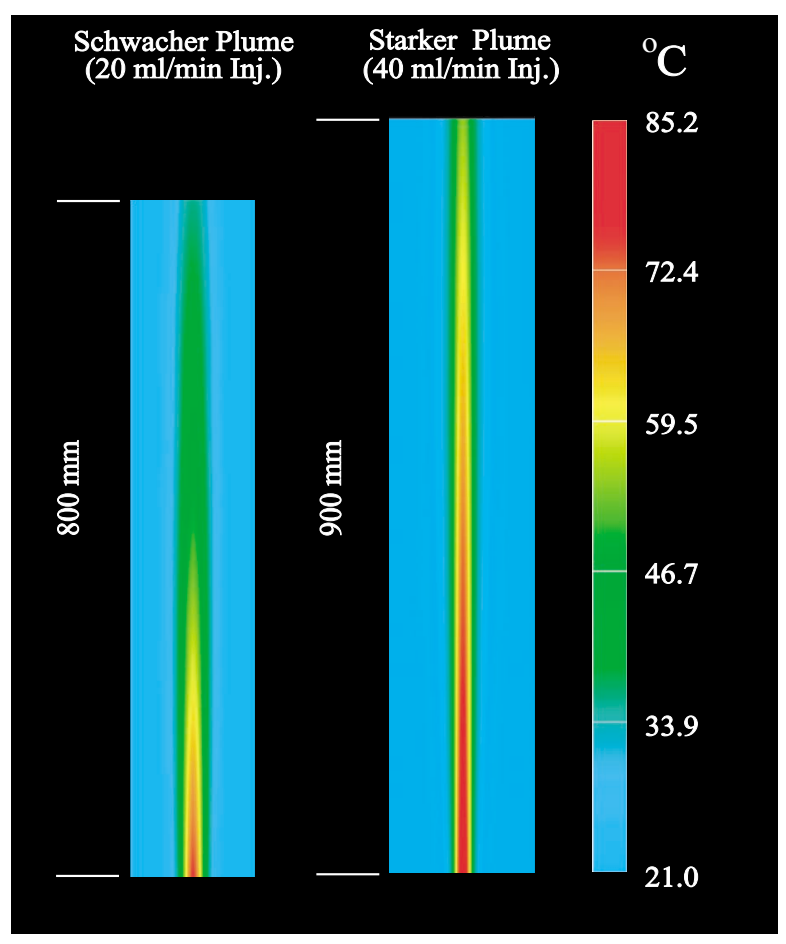

Abbildung 2.9: Beispiel für zwei vollständig ausgemessene Plumekanäle. Die dargestellten Temperaturfelder sind aus 17 bzw. 19 Einzelprofilen (gemessen ab einer Höhe von $15 \mathrm{~cm}$ im $5 \mathrm{~cm}$ Abstand) zusammengesetzt worden. Der schwächere Plume verbreitert sich deutlich auf seinem Weg nach oben und und kühlt durch thermische Diffusion wesentlich stärker aus als der Plume mit dem größeren Volumenfluß.

\subsection{Particle Image Velocimetry}

In diesem Kapitel wird auf die zur Geschwindigkeitsmessung verwendete Particle Image Velocimetry (kurz PIV-) Methode eingegangen. Es werden der verwendete Aufbau und das Prinzip erklärt, sowie die Auswertungsmethode erläutert und einige Beispielergebnisse präsentiert. Dabei sollen insbesondere die für das Plumeexperiment wichtigen Aspekte der Methode beschrieben werden. Ausführlichere und allgemeinere Darstellungen der Methode finden sich in Lehrbüchern der Strömungsmeßtechnik (z.B. Eckelmann, [1997] und Raffel et al., [1998]).

\subsubsection{Aufbau, Prinzip und Auswertungsmethode}

Die PIV-Methode ist ein Geschwindigkeitsfeldmeßverfahren, mit dem man stationäre und instationäre Strömungen berührungslos untersuchen kann. Dazu wird das Fluid mit lichtreflektierenden Partikeln versetzt und ein zweidimensionaler Bereich mit einem Lichtschnitt ausgeleuchtet. Senkrecht zu diesem werden Partikelbilder mit einer fotographischen oder ei- 
ner CCD-Kamera aufgenommen. Der prinzipielle Aufbau ist in Abbildung 2.10 dargestellt.

In der Regel werden zwei oder mehr Partikelbilder mit klar definierten zeitlichen Abständen zueinander durch Einfach- oder Mehrfachbelichtung aufgenommen und anschließend die Verschiebung der Teilchen durch Korrelationstechniken zwischen den aufgenommenen Strukturen bestimmt. Dazu werden die Bilder in sogenannte Abtastflecken (engl.: interrogation spots) unterteilt, in denen die Teilchengeschwindigkeiten möglichst wenig um einen Mittelwert streuen sollten, da das Ergebnis der Korrelation die mittlere Verschiebung der sich in diesem Fleck befindlichen Teilchenabbilder bestimmt. Die Größe der Abtastflecken ist durch die kleinste in der Strömung noch aufzulösende Struktur bestimmt.

Bei der hier verwendeten PIV-Methode sind die Anforderungen im Vergleich zu anderen strömungsmechanischen Anwendungen einerseits relativ gering, da die Strömung nicht turbulent ist und die Geschwindigkeiten mit maximal $50 \mathrm{~mm} / \mathrm{s}$ vergleichsweise klein sind. AuBerdem ist die Strömung bei einem Schnitt durch das Plumezentrum theoretisch zweidimensional, so daß es keine Geschwindigkeitskomponente normal zum Lichtschnitt gibt. Dadurch wandern keine Teilchen senkrecht aus der beleuchteten Ebene heraus oder in sie hinein. Andererseits können aber die sehr steilen Geschwindigkeitsgradienten zwischen Plumezentrum und Umgebungsmaterial unter Umständen Probleme bereiten, die sich aber durch die nachfolgend beschriebene Auswertungsmethode beheben lassen. Des weiteren sind die bei der Wellenausbreitung auftretenden maximalen Vertikalgeschwindigkeiten um mehrere Größenordnungen größer als die maximalen Horizontalgeschwindigkeiten, weshalb in x-Richtung deutlich kleinere Teilchenverschiebungen auftreten als in y-Richtung. Dadurch kann es zu Auflösungsproblemen der verhältnismäßig kleinen Verschiebungen in x-Richtung kommen.

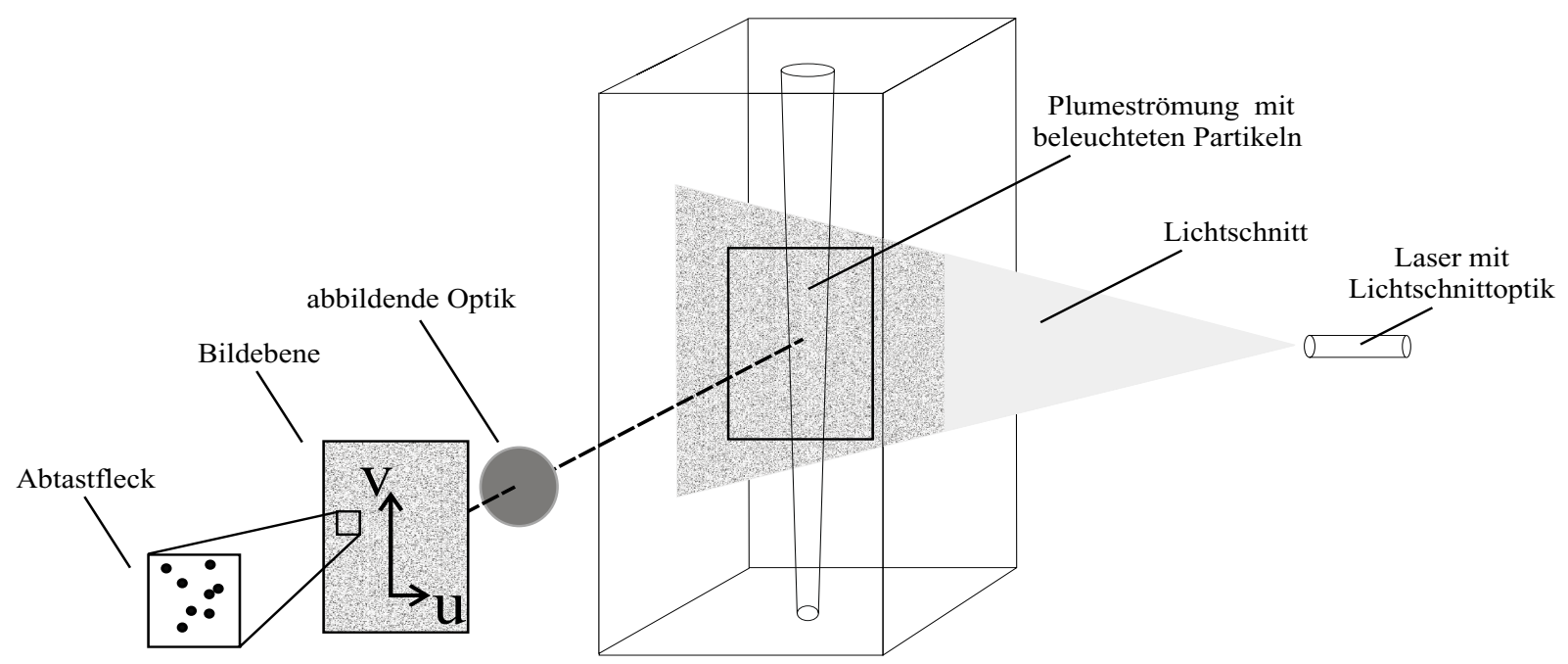

Abbildung 2.10: Prinzipieller Aufbau der PIV-Meßmethode. Das Rechteck im Lichtschnitt markiert den durch die Kamera beobachteten Bereich. Bei einem Schnitt durch das Plumezentrum treten nur die Geschwindigkeitskomponenten $\mathrm{u}$ und $\mathrm{v}$ auf. 
In dem hier dargestellten Experiment sind immer jeweils zwei Einzelbelichtungen auf unterschiedlichen Bildern aufgenommen worden (engl.: double frame/single exposure recording). Zur Auswertung wird daher eine Kreuzkorrelation zwischen den Intensitätsverteilungen der beiden Bilder verwendet. Da diese bei einer digitalen Aufzeichnung bzw. einer nachträglichen Digitalisierung analoger Bilder in Form von Pixeln vorliegen, benötigt man die diskrete Kreuzkorrelationsfunktion

$$
R(x, y)=\sum_{i, j} \operatorname{Int}_{1}(i, j) \operatorname{Int}_{2}(i+x, j+y),
$$

wobei $i$ und $j$ die Pixelnummern der zu korrelierenden Intensitäten der Abtastflecken $\operatorname{Int}_{1}(x, y)$ und $\operatorname{Int}_{2}(x, y)$ darstellen.

Gibt es eine Korrelation zwischen den beiden Teilbildern, so liefert $R(x, y)$ im Idealfall eine einzelne Spitze, deren relative Lage zum Zentrum des ersten Teilbildes die mittlere Verschiebung der in den Teilbildern enthaltenen Partikel angibt. Zur Rechenzeitreduzierung wird in der Regel vom Faltungssatz Gebrauch gemacht, der besagt, daß die Fourier-Transformierte der Faltung zweier Funktionen gleich dem Produkt der Fourier-Transformierten der Einzelfunktionen ist. Für die PIV-Auswertung heißt das, daß die Korrelationsfunktion $R$ im allgemeinen nicht mit Gleichung 2.8, sondern durch die Multiplikation von $\operatorname{Int}_{1}(i, j)$ mit dem komplex Konjugierten von $\operatorname{Int}_{2}(i, j)$ im Frequenzraum und einer anschließenden Rücktransformation des Produkts in den Ortsraum berechnet wird. Abbildung 2.11 stellt schematisch einen solchen Auswertevorgang mit Hilfe einer Kreuzkorrelation dar. Um anstelle von normalen Fourier-Transformationen schnelle Fourier-Transformationen (FFT) nutzen zu können, benötigt man in den meisten Fällen Teilbilder der Größe $N \times N$ mit $N=2^{n}$, $n=2,3,4,5, \ldots$. Alternativ lassen sich Teilbilder mit davon abweichenden Dimensionen auch durch ein Auffüllen mit Nullen (engl.: zero padding, z.B. Ronneberger et al., [1998]) auf diese Dimensionen bringen.

In Abbildung 2.12 sind zwei im Experiment aufgenommene Beispielabtastflecken und die daraus berechnete Korrelationsfunktion dargestellt. Da die Auftragung unnormiert ist, hat die Skalierung der vertikalen Achse keine Bedeutung. Um die genaue Lage der Korrelationsspitze zu bestimmen, wird diese in x- und y-Richtung mit einem Dreipunktsfit angepaßt (z.B. Gaußsche Anpassung). Daraus läßt sich dann die Position mit einer „Subpixel“-

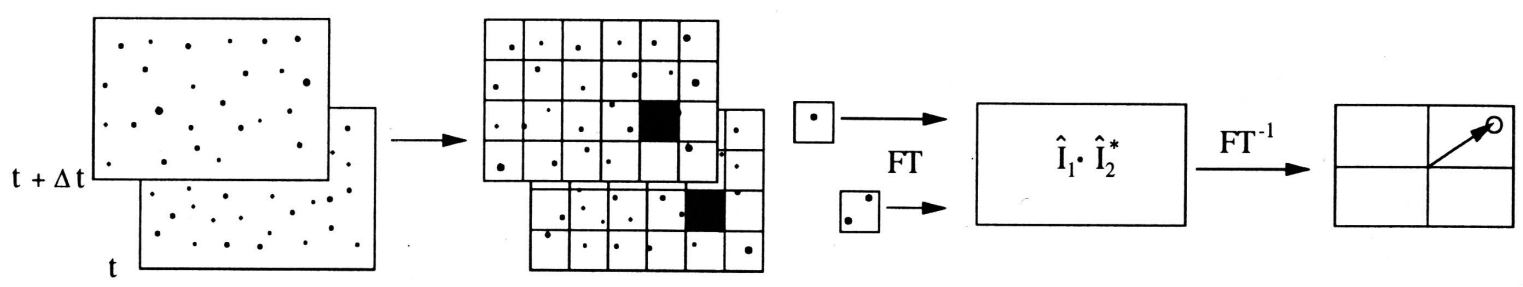

Abbildung 2.11: Schematische Darstellung einer PIV-Auswertung von zwei Einzelbelichtungen mit Hilfe einer Kreuzkorrelation (aus Raffel et al., [1998]) 

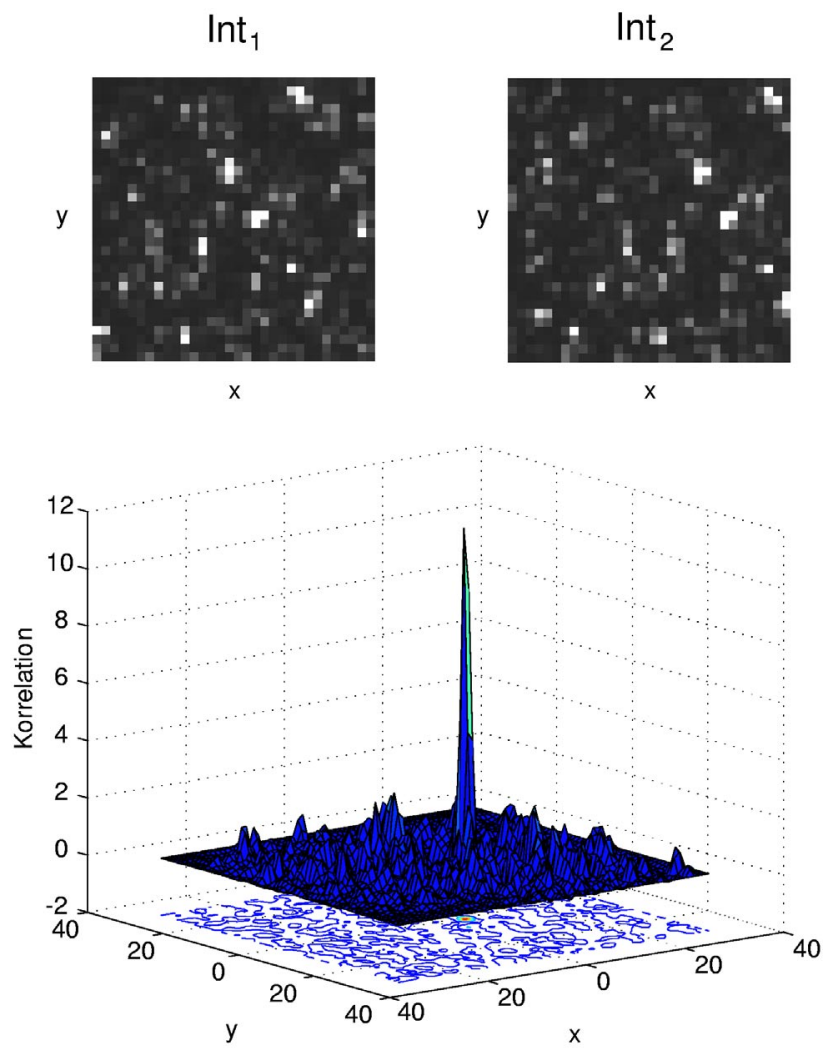

Abbildung 2.12: Beispielhaft herausgegriffene Abtastflecken mit zugehöriger unnormierter Korrelationsfunktion.

Genauigkeit (Raffel et al., [1998]) abschätzen. Die mittlere Verschiebung für das Beispiel in Abbildung 2.12 beträgt in x-Richtung 6,85 Pixel und in y-Richtung -0,25 Pixel. Der komplette Vorgang wird für alle Abtastflecken der Bilder wiederholt, so daß sich anschließend mit dem bekannten Bildmaßstab und dem zeitlichen Abstand zwischen den Aufnahmen die mittleren Teilchengeschwindigkeiten $\mathrm{u}(\mathrm{x}, \mathrm{y})$ und $\mathrm{v}(\mathrm{x}, \mathrm{y})$ in den Flecken berechnen lassen. Offensichtlich falsch bestimmte Geschwindigkeitskomponenten werden so weit wie möglich herausgefiltert (vergl. Westerweel, [1994]) und durch Interpolation der Werte an den Nachbarpunkten neu berechnet.

In der hier vorliegenden Arbeit wird für die Auswertung kein kommerzielles, sondern ein speziell auf die Anforderungen des Plumeexperiments zugeschnittenes Programm verwendet. Um die starken Geschwindigkeitsgradienten in horizontaler Richtung behandeln zu können, ist es möglich, den Abtastflecken des zweiten Bildes eine diskrete Vorverschiebung in vertikaler Richtung in ganzzahligen Pixeln in Abhängigkeit von der Horizontalkoordinate $x$ zu verleihen. Dadurch können zum einen die Abtastflecken kleiner gewählt werden, was die Geschwindigkeitsgradienten in diesem ausgewählten Bereich reduziert, zum anderen verbessert sich dadurch auch die Schärfe der Korrelationsspitze, weil weniger Teilchenpaare durch ein Herauswandern aus den Abtastflecken verloren gehen. Westerweel et al. [1997] zeigen mit Hilfe von theoretischen Studien, daß eine derartige Maßnahme auch das Signal-Rausch- 
Verhältnis verbessern kann. Im Zusammenhang mit der Bestimmung der kleinen horizontalen Geschwindigkeiten bei der Wellenausbreitung hat sich gezeigt, daß eine Vorverschiebung in vertikaler Richtung aufgrund der genannten Punkte unverzichtbar ist.

Als Vorverschiebungsdaten werden „geschätzte“ horizontale Geschwindigkeitsprofile in diskretisierter Form verwendet. Diese stammen entweder aus vorläufigen Auswertungen mit sehr großen Abtastflecken und entsprechend groben Schätzungen für die Verschiebungen oder zum Teil auch aus den Ergebnissen der in Kapitel 3 beschriebenen numerischen Modellierung.

Das vollständig in C geschriebene Auswerteprogramm verwendet die FFT-Routinen von Frigo und Johnson, www.fftw.org sowie weitere frei erhältliche C-Routinen zum Verarbeiten von 8-bit Tiff-Bildern. Es ermöglicht eine beliebig starke Überlappung der Korrelationsflecken, ist aber meistens mit einem 50\%-igen Überlapp verwendet worden.

\subsubsection{Experiment und Beispielergebnisse}

Für die experimentelle Umsetzung der PIV-Methode sind dem Sirup silberbeschichtete hohle Glaskugeln mit einer Dichte von $1,4 \mathrm{~g} / \mathrm{cm}^{3}$ und einer mittleren Größe von $10 \mu \mathrm{m}$ beigemischt worden. Für die Bildaufnahme ist zunächst mit einer fotografischen Technik und einem $100 \mathrm{~mW}$ Diodenlaser gearbeitet worden. Dazu sind jeweils zwei Partikelbilder mit einer durch eine Steuerung automatisch ausgelösten Spiegelreflexkamera aufgenommen worden. Bei dieser Technik beträgt der minimale Abstand zwischen zwei Aufnahmen etwa 0,3 s. Dieser Wert ergibt sich hauptsächlich aus der notwendigen Belichtungszeit von 1/60 s und aus der für den Filmtransport erforderlichen Zeit. Nach der Belichtung sind die Filme je nach eingefallener Lichtintensität entwickelt und mit einen Diascanner digitalisiert worden. Dabei tritt das Problem auf, daß durch das Einscannen eine künstliche Teilchenverschiebung auftreten kann, weil die Negative nie exakt gleich im Scanner positioniert werden können. Die Bilder sind daher so aufgenommen worden, daß sie immer Bereiche mit verschwindender Geschwindigkeit enthalten haben, mit denen die durch die Digitalisierung erzeugten Verschiebungsanteile herauskorrigiert werden konnten. Eine weitere Schwierigkeit der fotographischen Methode liegt darin, daß sich der Sirup mit zunehmendem Alter bzw. Erhitzungszyklen allmählich verdunkelt, so daß die in die Kamera reflektierte Lichtintensität zwischen den Experimenten zunehmend geringer wird. Deshalb sind vor jeden Experiment Probebelichtungen durchgeführt worden, die diesen Effekt quantifiziert haben, so daß dieser durch entsprechend längere Filmentwicklungszeiten kompensiert werden konnte.

Abbildung 2.13 zeigt ein mit der fotographischen Methode bestimmtes Horizontalprofil der Vertikalgeschwindigkeit in einem Plumekanal mit einem Volumenfluß von $60 \mathrm{ml} / \mathrm{min}$ in einer Aufstiegshöhe von $630 \mathrm{~mm}$ und einem Anfangstemperaturkontrast von $66^{\circ} \mathrm{C}$.

Weitere Versuche haben gezeigt, daß die fotographische Methode generell in der Lage ist, relativ langsame Geschwindigkeiten in Plumekanälen zu bestimmen, aber insbesondere bei der Untersuchung von solitären Wellen schnell an ihre Grenzen stößt. Das liegt hauptsächlich daran, daß der zeitliche Abstand zwischen zwei Aufnahmen wegen der relativ langen 


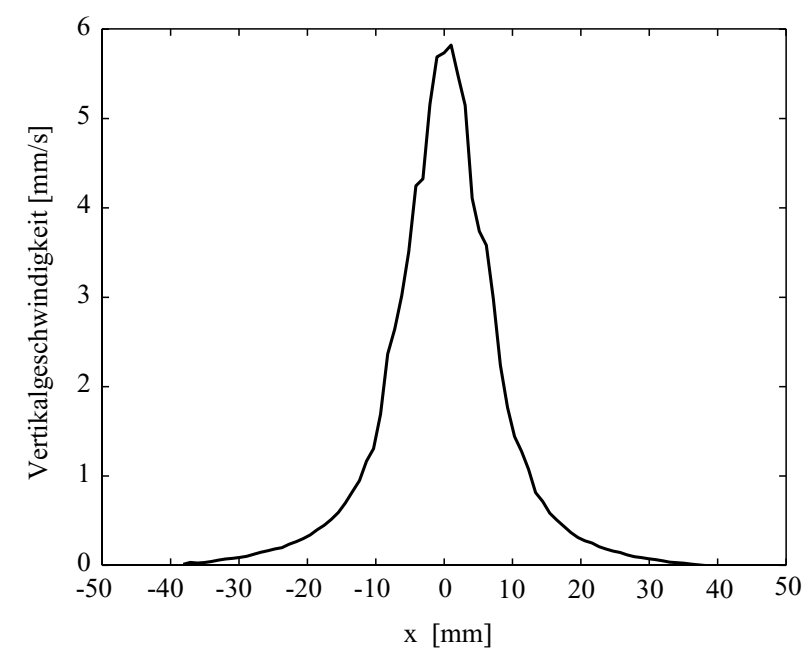

Abbildung 2.13: Fotographisch bestimmtes Horizontalprofil der Vertikalgeschwindigkeit in einem Plumekanal in $630 \mathrm{~mm}$ Aufstiegshöhe bei einem Anfangstemperaturkontrast von $66^{\circ} \mathrm{C}$ und einem Volumenfluß von $60 \mathrm{ml} / \mathrm{min}$.

Belichtungszeit von einer 1/60 s (bei geringer werdender Lichtintensität zum Teil auch länger) und dem mechanischen Filmtransport nicht ausreichend kurz gewählt werden kann. Unter Umständen ließe sich die Methode aber dadurch verbessern, daß man anstelle der zwei Einzelbelichtungen eine Doppeltbelichtung auf ein einzelnes Negativ (engl.: single frame/double exposure recording) durchführt. Dann müßte lediglich der verwendete Laser in der Lage sein, genügend Licht in zwei aufeinanderfolgenden Pulsen zu liefern. An die Stelle der Kreuzkorrelationsfunktion würde zur Bestimmung der Verschiebung die Autokorrelationsfunktion treten.

Da sich die fotographische Methode insgesamt zwar als kostengünstig, aber sehr aufwendig erwiesen hat, sind anschließend noch Messungen mit einer CCD-Kamera und einem durch Blitzlampen gepumpten $\mathrm{Nd}: \mathrm{YAG}^{1}$ Pulslaser mit Frequenzverdopplung durchgeführt worden (für technische Details der verwendeten Geräte vergl. Anhang A.4). Mit diesen Geräten ist der zeitliche Abstand zwischen zwei Aufnahmen auf 0,1 s verkürzt worden. Die durch die höhere Leistung des Nd:YAG-Lasers verbesserte Ausleuchtung hatte auch eine erhöhte Teilchendichte in den Aufnahmen zufolge, da selbst kleinste Partikel noch ausreichend viel Licht reflektiert haben, um von der CCD-Kamera registriert zu werden. Die Beispielteilbilder in Abbildung 2.12 sind mit dieser Versuchsanordnung aufgenommen worden.

In Abbildung 2.14 sind die Geschwindigkeiten in einem aufsteigenden Plumekopf dargestellt, die mit diesen Geräten gemessen worden sind. Da die Horizontalkomponente der Geschwindigkeit um zwei Größenordnungen kleiner ist als die Vertikalkomponente, wird auf die übliche Vektorpfeildarstellung verzichtet und stattdessen jede Geschwindigkeitskomponente getrennt voneinander als Konturdarstellung abgebildet.

In der Vertikalgeschwindigkeit erkennt man sehr gut die Bereiche des Plumekopfes, in

\footnotetext{
${ }^{1}$ Neodym-Yttrium-Aluminium-Granat
} 
denen das Umgebungsmaterial spiralartig eingebunden wird. Hier fällt die Geschwindigkeit auf Null ab, während sie weiter außen wieder ansteigt. In einem mit dem Plumekopf mitbewegten Koordinatensystem sind diese Bereiche durch einen Rückstrom gekennzeichnet. Die Horizontalkomponente ist so dargestellt, daß Geschwindigkeiten nach links ein positives und nach rechts ein negatives Vorzeichen haben. Man erkennt, daß das Material im oberen Bereich vom Plumezentrum stark nach außen wegströmt und weiter unten großräumig zum Zentrum zurückströmt. Im Ergebnisteil (Kapitel 4) sind weitere Resultate und Vergleiche mit numerischen Ergebnissen dargestellt.
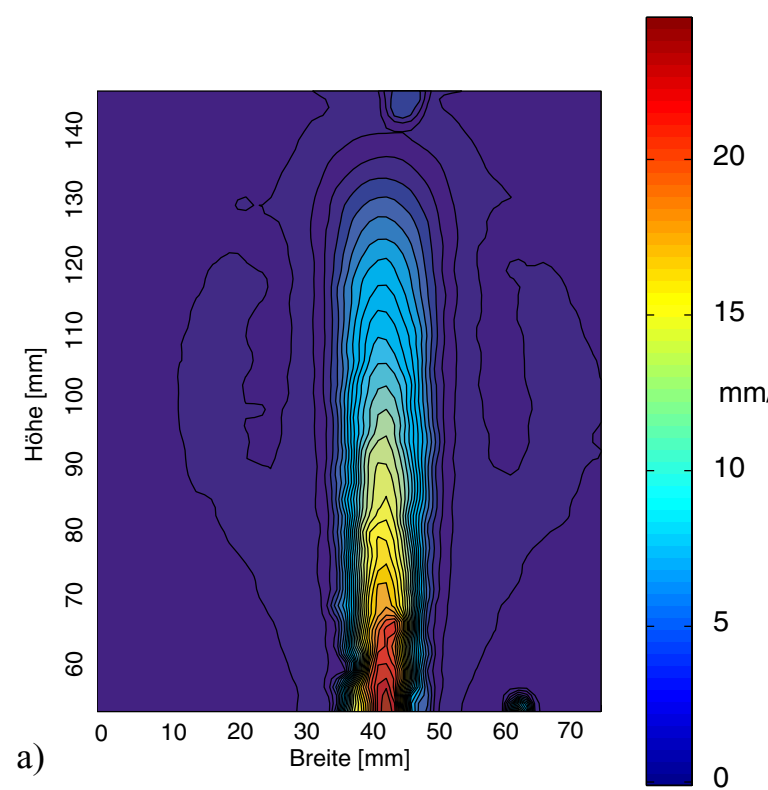

b)

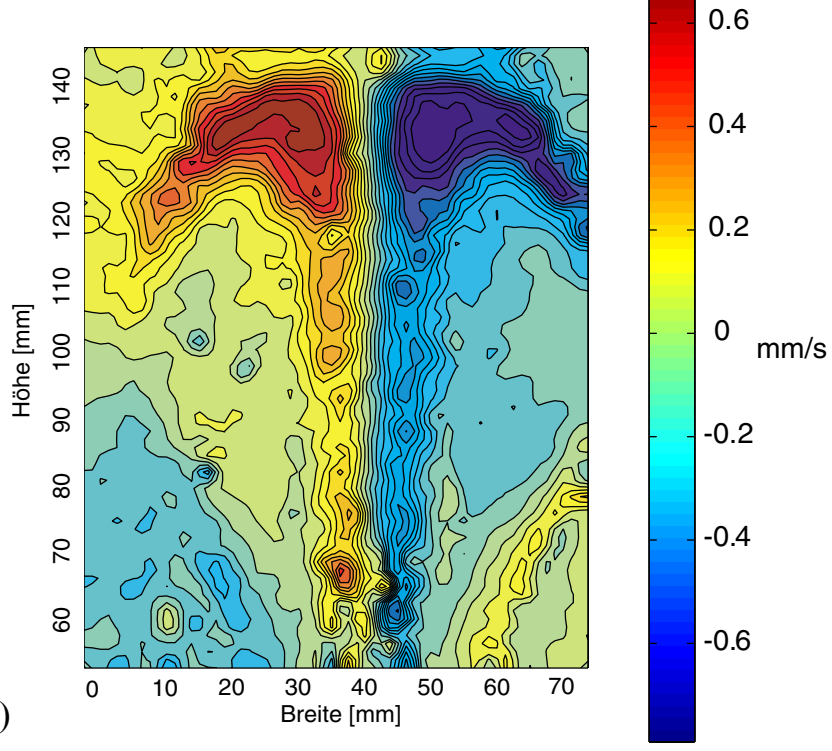

Abbildung 2.14: Vertikale (a) und horizontale Geschwindigkeitskomponente (b) eines aufsteigenden Diapirs. Die Vertikalkomponente zeigt deutlich den Bereich in denen das Umgebungsmaterial spiralartig eingebunden wird (vergl. Abbildung $2.2 \mathrm{a})$. 


\section{Kapitel 3}

\section{Numerische Modellierung}

Die hier beschriebene numerische Modellierung von thermischen Plumes ist mit zwei Zielsetzungen durchgeführt worden: Zunächst sollte das Laborexperiment möglichst exakt nachgebildet werden, um dann in einem zweiten Schritt auch Rechnungen mit erdähnlicheren Materialeigenschaften, die im Experiment nicht zugänglich sind, anzustellen zu können. Für das Modell ist eine zweidimensionale, axialsymmetrische Geometrie gewählt worden, da im Experiment keine oder nur geringfügige Variationen der physikalischen Größen in azimutaler Richtung zu beobachten sind. Auf die Plumes der Erde bezogen, stellt ein zweidimensionales Modell unter Umständen eine starke Vereinfachung dar, wenn die Quellregion des Plumes an einer thermischen Grenzschicht mit in die Modellierung einbezogen werden soll. Analog zum Experiment wird aber auch in der numerischen Modellierung die Quellregion der Plumes außer acht gelassen. Die Unterseite des Modellzylinders liegt in einiger Entfernung zu einer gedachten thermischen Grenzschicht, so daß der zweidimensionale Ansatz zwar eine Idealisierung aber eine gerechtfertigte Approximation darstellt. Außerdem hat die zweidimensionale Geometrie den Vorteil, daß die großen, für die Untersuchung von solitären Wellen erforderlichen, Rechengebiete nicht zu einem unvertretbar hohen Rechenaufwand, insbesondere bei systematischen Parameterstudien, führen.

Die folgenden Abschnitte beschreiben zunächst die physikalischen Grundgleichungen in Zylinderkoordinaten, sowie angebrachte Vereinfachungen bzw. Herleitungen, die für die numerische Umsetzung von Bedeutung sind. Dabei wird erst auf die Kontinuitäts- und die Stokes-Gleichung, sowie deren Skalierung und Umformung eingegangen. Anschließend folgt eine ähnliche Darstellung für die Wärmetransportgleichung. Danach werden die Randbedingungen beschrieben, die in Analogie zum Laborexperiment gewählt worden sind. Am Ende des Kapitels folgen Abschnitte, die die Diskretisierung und die Lösungsverfahren der Stokes- und Wärmetransportgleichung, das verwendete Gitter, sowie einige Programmtests beschreiben. 


\subsection{Physikalische Grundgleichungen}

In der Geodynamik ist es üblich, die Fließbewegungen des Mantelgesteins mit den Grundgleichungen der Hydrodynamik zu beschreiben. Über geologisch lange Zeitskalen läßt sich die Dynamik des Erdmantels analog zu einer hochviskosen Flüssigkeit behandeln (Turcotte und Schubert, [1982]). Die Prandtl-Zahl

$$
\operatorname{Pr}=\frac{v}{\kappa}
$$

beschreibt das Verhältnis zwischen viskosen Kräften und Trägheitskräften. Dabei ist $v=$ $\eta / \rho$ die kinematische Viskosität mit $\eta$ der dynamischen Viskosität und $\rho$ der Dichte und $\kappa=\lambda /\left(\rho c_{p}\right)$ die thermische Diffusivität mit der Wärmeleitfähigkeit $\lambda$ und der spezifischen Wärmekapazität bei konstantem Druck $c_{p}$. Setzt man für den Erdmantel repräsentative Werte $\left(\eta=10^{21} \mathrm{Pas}, \rho=4000 \mathrm{~kg} / \mathrm{m}^{3}, \kappa=10^{-6} \mathrm{~m}^{2} / \mathrm{s}\right)$ ein, so erhält man eine Prandtl-Zahl von $\operatorname{Pr} \approx 10^{23}$. Für das Laborexperiment ergibt sich mit den im Anhang A.1 angegebenen Werten ein $\operatorname{Pr} \approx 10^{6}$. In beiden Fällen überwiegt der Einfluß der viskosen Kräfte deutlich den Einfluß der Trägheitskräfte, so daß die Trägheitskräfte in den nachfolgend beschriebenen Gleichungen vernachlässigt werden können. Als weitere Vereinfachung findet die BoussinesqApproximation Anwendung, wonach alle Dichtevariationen in den Gleichungen grundsätzlich vernachlässigt werden und lediglich der Auftriebsterm erhalten bleibt.

\subsubsection{Kontinuitäts- und Stokes-Gleichung}

Die hier aufgeführten Gleichungen in Zylinderkoordinaten $(r, \phi, z)$ mit den dazugehörigen Geschwindigkeitskomponenten $(u, v, w)$ sind in dieser Form aus Schowalter [1978] entnommen.

Die Kontinuitätsgleichung lautet

$$
\frac{1}{r} \frac{\partial}{\partial r}(r u)+\frac{1}{r} \underbrace{\frac{\partial v}{\partial \phi}}_{=0}+\frac{\partial w}{\partial z}=0
$$

und die Komponenten der Stokesgleichung sind

$$
\begin{gathered}
r: \quad-\frac{\partial p}{\partial r}+\frac{1}{r} \frac{\partial}{\partial r}\left(r \tau_{r r}\right)+\frac{1}{r} \underbrace{\frac{\partial \tau_{r \phi}}{\partial \phi}}_{=0}-\frac{\tau_{\phi \phi}}{r}+\frac{\partial \tau_{r z}}{\partial z}=0 \\
\phi: \quad-\frac{1}{r} \frac{\partial p}{\partial \phi}+\frac{1}{r^{2}} \underbrace{\frac{\partial}{\partial r}\left(r^{2} \tau_{r \phi}\right)}_{=0}+\frac{1}{r} \frac{\partial \tau_{\phi \phi}}{\partial \phi}+\underbrace{\frac{\partial \tau_{\phi z}}{\partial z}}_{=0}=0 \\
z: \quad-\rho g-\frac{\partial p}{\partial z}+\frac{1}{r} \frac{\partial}{\partial r}\left(r \tau_{r z}\right)+\frac{1}{r} \underbrace{\frac{\partial \tau_{\phi z}}{\partial \phi}}_{=0}+\frac{\partial \tau_{z z}}{\partial z}=0,
\end{gathered}
$$


wobei $\mathrm{p}$ der Druck und die $\tau_{i j}$ die Anteile des deviatorischen Spannungstensors $\underline{\underline{\tau}}$ sind. Außerdem wird angenommen, daß der Schwerevektor $\vec{g}$ parallel zur z-Richtung ist, so daß $\vec{g}=(0,0,-g)$.

Aufgrund der Axialsymmetrie fallen die Azimutalkomponenten der Gleichungen 3.1, 3.2 und 3.4 sowie der azimutale Anteil der Stokesgleichung 3.3 weg.

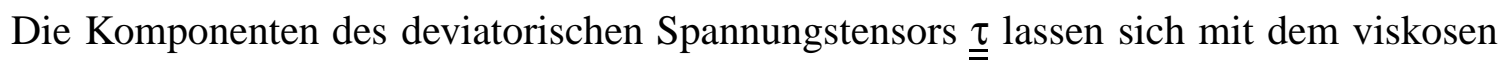
Fließgesetz (Jarvis und McKenzie, [1980])

$$
\underline{\underline{\tau}}=2 \eta\left(\underline{\underline{\dot{\varepsilon}}}-\frac{1}{3}(\vec{\nabla} \cdot \vec{v}) \underline{\underline{\delta}}\right)
$$

bestimmen, wobei $\eta$ die (Scher-) Viskosität, $\underline{\underline{\delta}}$ der Einheitstensor und $\underline{\underline{\varepsilon}}$ der symmetrische Deformationsratentensor ist. Die Komponenten dieses Tensors lauten in Zylinderkoordinaten (Batchelor, [1992])

$$
\begin{aligned}
\dot{\varepsilon}_{r r}=\frac{\partial u}{\partial r}, \dot{\varepsilon}_{\phi \phi} & =\frac{1}{r} \underbrace{\frac{\partial v}{\partial \phi}}_{=0}+\frac{u}{r}, \dot{\varepsilon}_{z z}=\frac{\partial w}{\partial z}, \\
\dot{\varepsilon}_{r \phi} & =\frac{r}{2} \frac{\partial}{\partial r} \underbrace{\left(\frac{v}{r}\right)}_{=0}+\frac{1}{2 r} \underbrace{\frac{\partial u}{\partial \phi}}_{=0}=0, \\
\dot{\varepsilon}_{\phi z} & =\frac{1}{2 r} \underbrace{\frac{\partial w}{\partial \phi}}_{=0}+\frac{1}{2} \underbrace{\frac{\partial v}{\partial z}}_{=0}=0, \\
\dot{\varepsilon}_{r z} & =\frac{1}{2} \frac{\partial u}{\partial z}+\frac{1}{2} \frac{\partial w}{\partial r},
\end{aligned}
$$

wobei aufgrund der angenommenen Axialsymmetrie die Komponenten $\dot{\varepsilon}_{r \phi}$ und $\dot{\varepsilon}_{\phi z} \mathrm{zu}$ Null werden. Da in diesem Modell eine inkompressible Strömung angenommen wird, gilt die Divergenzfreiheit des Geschwindigkeitsfeldes

$$
\vec{\nabla} \cdot \vec{v}=0
$$

so daß sich nach dem Fließgesetz 3.5 die nichtverschwindenden Anteile des deviatorischen Spannungstensors $\underline{\underline{\tau}}$ lediglich durch den Vorfaktor $2 \eta$ von den Anteilen des Deformationsratentensors $\underline{\underline{\varepsilon}}$ unterscheiden.

Der Druck $p$ setzt sich aus dem nur von $z$ abhängenden hydrostatischen Anteil $p_{h y d r}$ und dem nicht-hydrostatischen Anteil $\tilde{p}$ zusammen, so daß sich

$$
\frac{\partial p}{\partial r}=\frac{\partial \tilde{p}}{\partial r} \text { und } \frac{\partial p}{\partial z}=\frac{\partial p_{h y d r}}{\partial z}+\frac{\partial \tilde{p}}{\partial z}=-\rho_{0} g+\frac{\partial \tilde{p}}{\partial z}
$$

als Ableitungen des Drucks ergeben, wobei $\rho_{0}=\rho\left(T_{0}\right)$ die Dichte des Materials bei der Referenztemperatur $T_{0}$ ist. Zusammen mit den Druckableitungen 3.11 und der Zustandsgleichung

$$
\rho=\rho_{0}\left(1-\alpha\left(T-T_{0}\right)\right)
$$


die über den thermischen Ausdehnungskoeffizienten $\alpha$ die Temperaturabhängigkeit der Dichte $\rho$ beschreibt, ergibt sich für die verbleibenden Anteile der Stokesgleichung 3.2 und 3.4

$$
\begin{aligned}
& r: \quad-\frac{\partial \tilde{p}}{\partial r}+\frac{1}{r} \frac{\partial}{\partial r}\left(r \tau_{r r}\right)-\frac{\tau_{\phi \phi}}{r}+\frac{\partial \tau_{r z}}{\partial z}=0 \\
& z: \rho_{0} g \alpha\left(T-T_{0}\right)+\frac{1}{r} \frac{\partial}{\partial r}\left(r \tau_{r z}\right)-\frac{\partial \tilde{p}}{\partial z}+\frac{\partial \tau_{z z}}{\partial z}=0 .
\end{aligned}
$$

\section{Skalierung und Vereinfachung}

Um die Anzahl der freien Parameter in den Gleichungen zu reduzieren, ist es allgemein üblich, die dimensionsbehafteten Größen durch dimensionslose zu ersetzen. Als Längenskala wird hier der Radius des Modellzylinders $R_{0}$ verwendet, so daß

$$
r=r^{\prime} \cdot R_{0} \text { und } z=z^{\prime} \cdot R_{0} .
$$

Dabei und im folgenden sind die dimensionslosen Größen mit Strichen $\left(^{\prime}\right)$ versehen. Des weiteren wird

$$
\begin{gathered}
\tau=\tau^{\prime} \frac{\kappa \eta_{0}}{R_{0}^{2}}, \tilde{p}=\tilde{p}^{\prime} \frac{\kappa \eta_{0}}{R_{0}^{2}} \text { und } \\
T=T^{\prime} \cdot \Delta T+T_{0}
\end{gathered}
$$

zur Skalierung der Stokes-Gleichung verwendet. Hier ist $\Delta T$ die Temperaturanomalie des Plumematerials und $\eta_{0}$ die Referenzviskosität. Die dimensionslosen Anteile der StokesGleichung lauten dann:

$$
\begin{aligned}
& r: \quad-\frac{\partial \tilde{p}^{\prime}}{\partial r^{\prime}}+\frac{\tau_{r r}^{\prime}}{r^{\prime}}+\frac{\partial \tau_{r r}^{\prime}}{\partial r^{\prime}}-\frac{\tau_{\phi \phi}^{\prime}}{r^{\prime}}+\frac{\partial \tau_{r z}^{\prime}}{\partial z^{\prime}}=0 \\
& z: \underbrace{\frac{\rho_{0} g \alpha \Delta T R_{0}^{3}}{\kappa \eta_{0}}}_{R a} T^{\prime}-\frac{\partial \tilde{p}^{\prime}}{\partial z^{\prime}}+\frac{\partial \tau_{r z}^{\prime}}{\partial r^{\prime}}+\frac{\tau_{r z}^{\prime}}{r^{\prime}}+\frac{\partial \tau_{z z}^{\prime}}{\partial z^{\prime}}=0 .
\end{aligned}
$$

Nach der Skalierung enthält die Gleichung 3.19 als einzigen freien Parameter die mit dem Zylinderradius $R_{0}$ definierte Rayleighzahl $R a$. Da $R a$ normalerweise über die Höhe der konvektierenden Schicht $h$ definiert ist und in diesem Fall die Konvektion nicht an einer thermischen Grenzschicht, sondern durch Injizieren von heißem Material entsteht, ist es schwierig, dieser speziellen Rayleighzahl die übliche physikalische Bedeutung zuzuschreiben. In dieser Form stellt sie lediglich eine modellinterne Größe dar und sollte deshalb nicht ohne weiteres zum Vergleich mit anders definierten Rayleighzahlen herangezogen werden.

Zur Vereinfachung wird nun aus den Gleichungen der Druck eliminiert, indem 3.18 partiell nach $\mathrm{z}$ und 3.19 partiell nach $\mathrm{r}$ abgeleitet und anschließend die zweite von der ersten 
Gleichung subtrahiert wird. Die Terme der verbleibenden Gleichung, in der die dimensionslosen Größen von nun an der Übersichtlichkeit halber ohne Striche dargestellt sind,

$$
\begin{aligned}
\frac{\partial}{\partial z} \frac{\tau_{r r}}{r}+\frac{\partial^{2}}{\partial r \partial z} \tau_{r r} & -\frac{\partial}{\partial z} \frac{\tau_{\phi \phi}}{r}+\frac{\partial^{2}}{\partial z^{2}} \tau_{r z} \\
& -\frac{\partial^{2}}{\partial r^{2}} \tau_{r z}-\frac{\partial}{\partial r} \frac{\tau_{r z}}{r}-\frac{\partial^{2}}{\partial z \partial r} \tau_{z z}-R a \frac{\partial}{\partial r} T=0,
\end{aligned}
$$

lassen sich zum Teil noch zusammenfassen und die Komponenten des deviatorischen Spannungstensors, die sich aus den Gleichungen 3.6 und 3.9 mit 3.5 und 3.10 ergeben, einsetzen. Damit folgt

$$
\begin{gathered}
\frac{\partial^{2}}{\partial z^{2}}\left(\eta\left(\frac{\partial u}{\partial z}+\frac{\partial w}{\partial r}\right)\right)-\frac{\partial^{2}}{\partial r^{2}}\left(\eta\left(\frac{\partial u}{\partial z}+\frac{\partial w}{\partial r}\right)\right)+2 \frac{\partial^{2}}{\partial r z}\left(\eta\left(\frac{\partial u}{\partial r}-\frac{\partial w}{\partial z}\right)\right) \\
+\frac{2}{r} \frac{\partial}{\partial z}\left(\eta\left(\frac{\partial u}{\partial r}-\frac{u}{r}\right)\right)-\frac{\partial}{\partial r}\left(\frac{\eta}{r}\left(\frac{\partial u}{\partial z}+\frac{\partial w}{\partial r}\right)\right)-R a \frac{\partial}{\partial r} T=0 .
\end{gathered}
$$

Unter Verwendung der skalaren Stromfunktion $\psi$ lassen sich die beiden Geschwindigkeitskomponenten als

$$
u=-\frac{1}{r} \frac{\partial \psi}{\partial z} \quad \text { und } \quad w=\frac{1}{r} \frac{\partial \psi}{\partial r}
$$

ausdrücken, was sich direkt aus der Kontinuitätsgleichung 3.1 ergibt. Für die numerische Modellierung sind die Geschwindigkeiten in der Stokesgleichung 3.21 durch diese Ausdrücke 3.22 ersetzt worden, was den Vorteil hat, daß die Kontinuitätsgleichung nicht mehr gelöst werden muß, sondern a priori erfüllt ist. Der Nachteil liegt in der Erhöhung der Ordnung der Gleichung, was diese komplizierter und unübersichtlicher werden läßt, so daß hier auf eine Darstellung verzichtet und stattdessen auf den im Anhang A.2 dargestellten Ausdruck A.3 verwiesen wird.

\section{Viskositätsgesetz}

In der numerischen Modellierung wird das Viskositätsgesetz 2.1 des im Experiment verwendeten Glukosesirups verwendet. In dimensionsloser Form lautet es:

$$
\eta^{\prime}=\frac{\eta}{\eta_{0}}=\exp \left(\frac{1}{a+b T}-\frac{1}{a+b T_{0}}\right) \text { mit } \eta_{0}=\eta\left(T_{0}\right)
$$

In Rechnungen, in denen der Einfluß einer tiefenabhängigen Viskosität untersucht werden soll, wird Gleichung 3.23 mit der Exponentialfunktion

$$
f(z)=\exp \left(c_{v i s c}\left(z_{1 / 2}-z\right)\right)
$$

multipliziert. Die Konstante $c_{\text {visc }}$ gibt den Betrag der Viskositätszunahme mit der Tiefe an. In halber Zylinderhöhe bei $z=z_{1 / 2}$ ist $f(z)=1$. 
Während die bisher dargestellten Gleichungen das Gleichgewicht zwischen viskosen Kräften und Auftriebskräften beschreiben, wird mit der im folgenden dargestellten Wärmetransportgleichung die Temperaturänderung durch Diffusion und Advektion und damit auch die zeitliche Entwicklung des Systems formuliert. Zusammen mit der Stokes-Gleichung und den später beschriebenen Randbedingungen stellt sie ein gekoppeltes nichtlineares Differentialgleichungssystem dar, dessen Lösungsmethoden in Kapitel 3.3 beschreiben werden.

\subsubsection{Wärmetransportgleichung}

In der Wärmetransportgleichung

$$
\frac{\partial T}{\partial t}+\vec{v} \cdot \vec{\nabla} T=\kappa \vec{\nabla}^{2} T
$$

ist die Temperaturänderung durch adiabatische Expansion oder Kompression des Materials und durch dissipative Reibungswärme vernachlässigt worden, da diese im Laborexperiment keine Rolle spielt und im Erdmantel nur einen zweitrangigen Effekt darstellt. Die Gleichung wird analog zur Stokes-Gleichung skaliert. Neben den Ausdrücken 3.15 und 3.17 werden

$$
\begin{aligned}
\text { die Geschwindigkeitsskalierung } \vec{v} & =\vec{v}^{\prime} \frac{\kappa}{R_{0}} \\
\text { und die Zeitskalierung } \quad t & =t^{\prime} \frac{R_{0}^{2}}{\kappa}
\end{aligned}
$$

verwendet. In Zylinderkoordinaten lautet die dimensionslose Wärmetransportgleichung dann

$$
\frac{\partial T}{\partial t}=\frac{\partial^{2} T}{\partial r^{2}}+\frac{\partial^{2} T}{\partial z^{2}}+\frac{1}{r} \frac{\partial T}{\partial r}-u \frac{\partial T}{\partial r}-w \frac{\partial T}{\partial z}
$$

wobei auch hier die Striche an den dimensionslosen Größen weggelassen worden sind.

\subsection{Anfangs- und Randbedingungen}

Für die korrekte Formulierung des mathematischen Problems benötigt man für die StokesGleichung 3.21 bzw. A.3 jeweils zwei mechanische Randbedingungen auf den Rändern des Definitionsgebietes. Für die Wärmetransportgleichung 3.28 wird jeweils eine Temperaturrandbedingung auf den Rändern, sowie eine Temperaturanfangsbedingung $T_{t=0}(r, z)$ an jedem Punkt des Rechengebietes benötigt.

Die Randbedingungen die im Zusammenhang mit dem Laborexperiment gewählt worden sind, sehen wie folgt aus: 


\subsubsection{Unterseite}

An der Unterseite des Zylinders bei $z=0$ ist ein offener Rand angenommen worden, so daß heißes Material, dessen Temperatur über eine gaußörmige Temperaturrandbedingung $T(r, z=0)=\exp \left(-\left(r / r_{T}\right)^{2}\right)$ festgelegt ist, einströmen kann. Dabei legt die Konstante $r_{T}$ die Breite der gaußförmigen Temperaturrandbedingung fest. Der Volumenstrom $Q$ wird über eine ebenfalls gaußförmig vorgegebenen Einströmgeschwindigkeit $w(r, z=0)=$ $w_{0} \exp \left(-\left(r / r_{w}\right)^{2}\right)$ variiert. Dabei wird der Parameter $r_{w}$ konstant gehalten und $w_{0}$ gemäß

$$
Q=2 \pi \int_{0}^{1} r w_{0} \exp \left(-\left(r / r_{w}\right)^{2}\right) d r
$$

je nach gewünschtem Volumenfluß $Q$ verändert. Der hier dargestellte Volumenfluß $Q$ ist dimensionslos und läßt sich durch Multiplikation mit $\kappa \cdot R_{0}$ in den dimensionsbehafteten Volumenfluß umrechnen.

In der Abbildung 3.1 zeigt die durchgezogene Kurve die mit dem Maximalwert normierte dimensionslose Einströmgeschwindigkeit und die gestrichelte Kurve die dimensionslose Temperaturrandbedingung $T(r, z=0)$. Dabei ist der Parameter mit $r_{w}=0.02$ so gewählt, daß die Breite der Kurve ungefähr mit dem entdimensionalisiert eingezeichneten Radius $r_{D}$ der Injektionsöffnung des Laborexperiments im Einklang steht. Die Temperaturrandbedingung ist mit einem $r_{T}=0.09$ so breit gewählt worden, daß das einströmende Material eine annähernd konstante Temperatur aufweist. Auf das Experiment bezogen, fallen die Gaußfunktionen zum Rand hin nicht nicht steil genug ab, um die Einströmverhältnisse im Experiment exakt zu beschreiben. Es hat sich aber gezeigt, daß die Randbedingungen schon in geringem Abstand zur Zylinderunterseite keinen Einfluß mehr auf das Gesamtsystem haben, so

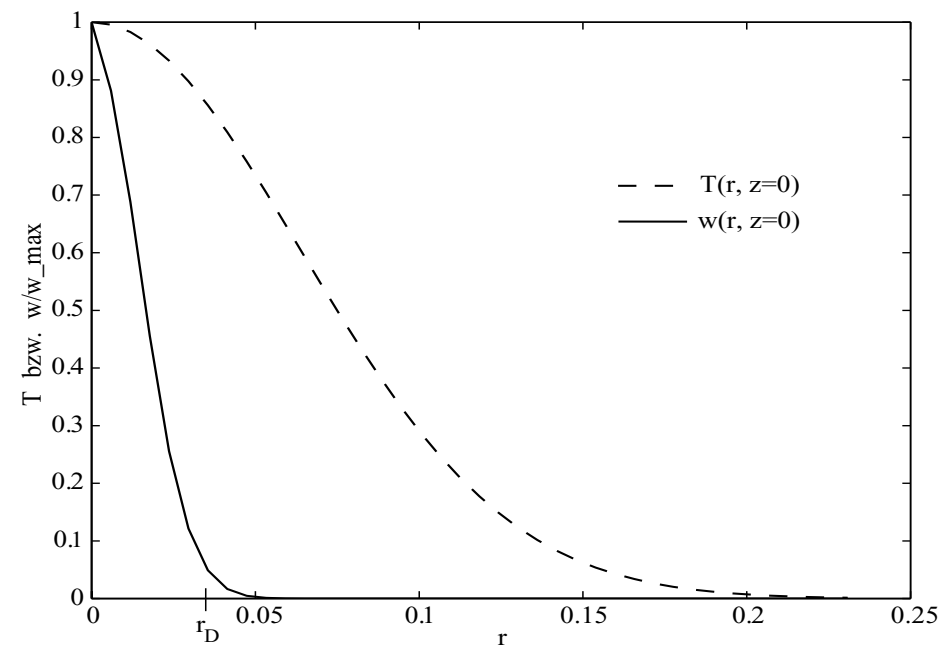

Abbildung 3.1: Temperaturrandbedingung (gestrichelt) und auf Eins normierte vertikale Einströmgeschwindigkeit (durchgezogen) an der Zylinderunterseite. $r_{D}$ kennzeichnet den Radius der Einströmöffnung im Laborexperiment. 
daß hier, auch um numerische Probleme zu vermeiden, keine Funktionen mit noch steileren radialen Temperatur- bzw. Geschwindigkeitsgradienten verwendet worden sind.

Für die Erzeugung von Volumenstörungen kann die Einströmgeschwindigkeit über ein zeitabhängiges $w_{0}(t)$ variiert werden. Konkret ist für die Wellenerzeugung $w_{0}$ für einen festgelegten Zeitraum stufenartig erhöht und anschließend wieder auf den Ausgangswert zurückgesetzt worden.

Aus dem radialen Einströmprofil $w(r, z=0)$ wird nach 3.22 durch Integration in radialer Richtung die Stromfunktion an der Unterseite $\psi(r, z=0)$ bestimmt, welche die eine der beiden mechanischen Randbedingungen darstellt. Als weitere Randbedingung wird

$$
\frac{\partial \psi}{\partial z}=0
$$

angenommen, was sich aus der Annahme einer verschwindenden radialen Geschwindigkeit $u(r, z=0)=0$ am Unterrand und dem Ausdruck für die Stromfunktion 3.22 ergibt.

\subsubsection{Außenrand}

Der Außenrand des Zylinder ist geschlossen, so daß kein Material hinein- oder hinausströmen kann, d.h. $u=0$ bzw. $\psi=0$ ist. Des weiteren kann zwischen einer Gleit- (engl.: freeslip) oder einer Haftrandbedingung (noslip) gewählt werden, die bestimmt, ob die vertikalen Spannungen $\tau_{r z}$ oder die Vertikalgeschwindigkeiten an der Gefäßwandung verschwinden. Im Zusammenhang mit dem Laborexperiment ist eine Haftrandbedingung realistischer. Es hat sich aber gezeigt, daß die Plumeströmung so weit vom Außenrand entfernt ist, daß die Vertikalgeschwindigkeiten unabhängig von der Wahl der Randbedingung auf Null abfallen. Daher ist es für dieses Modell unerheblich, mit welcher der beiden Varianten gerechnet wird. Tatsächlich sind die meisten Rechnungen mit der freeslip-Randbedingung $\tau_{r z}=0$ durchgeführt worden. Das bedeutet, das nach Gleichung 3.5 und 3.9 der Ausdruck

$$
\left(\frac{\partial u}{\partial z}+\frac{\partial w}{\partial r}\right)
$$

zu Null werden muß. Da $\partial u / \partial z=0$ wegen $u=0$ auf dem Außenrand ist, bleibt als Bedingung für die Stromfunktion

$$
\frac{\partial}{\partial r}\left(\frac{1}{r} \frac{\partial \psi}{\partial r}\right)=0 \text { bzw. }\left(\frac{\partial^{2} \psi}{\partial r^{2}}-\frac{1}{r} \frac{\partial \psi}{\partial r}\right)=0 .
$$

Als Temperaturrandbedingung ist eine verschwindende radiale Ableitung $\partial T / \partial r=0$ angesetzt worden.

\subsubsection{Oberseite}

Die Oberseite des Zylinders ist offen und spannungsfrei (d.h. $\tau_{z z}=0$ ), so daß die gleiche Materialmenge, die unten einströmt, oben aus dem Zylinder ungehindert wieder herausströmen kann. Dafür wird genau wie an der Unterseite die radiale Geschwindigkeit $u$ auf Null 
bzw. $\partial \psi / \partial z=0$ gesetzt. Für diese im Vergleich zum Experiment etwas künstliche Randbedingung wird außerdem angenommen, daß die dritte Ableitung von $\psi$ in vertikaler Richtung verschwindet. Der Unterschied zwischen Experiment und numerischen Modell besteht bei dieser Randbedingung darin, daß das heiße Material im Experiment die Plexiglassäule nicht verlassen kann und sich stattdessen an der Flüssigkeitsoberfläche sammelt und dort langsam auskühlt. Da dieses Verhalten jedoch keinen Einfluß auf die Plumeströmung in einiger Entfernung zur Flüssigkeitsoberfläche hat, besteht auch in numerischer Hinsicht keine Notwendigkeit, dieses zu berücksichtigen. Anschaulich kann man sich das numerisch modellierte Gebiet auch als einen Teilausschnitt aus dem Laborexperiment vorstellen.

Als Temperaturrandbedingung wird eine verschwindende vertikale Temperaturableitung $\partial T / \partial z=0$ gewählt.

\subsubsection{Zylinderachse}

Obwohl die Achse $r=0$ geometrisch keinen Rand des Zylinders darstellt, müssen hier dennoch Randbedingungen für das Rechengebiet aufgestellt werden. Diese ergeben sich hauptsächlich aus Symmetrieüberlegungen. So sollte hier die Radialgeschwindigkeit $u$ verschwinden bzw. kein Material durch das Zentrum hindurchfließen, so daß die Stromfunktion $\psi(r=$ $0, z)=$ const. sein muß. Damit kein Widerspruch zu der an der Unterseite angenommenen Randbedingung 3.30 entsteht, wird der konkrete Wert auf $\psi(r=0, z)=\psi(r=0, z=0)$ gesetzt. Weiterhin dürfen keine vertikalen Scherspannungen $\tau_{r z}$ vorkommen, so daß $\partial w / \partial r=0$ ist und mit 3.22 folgt, daß der Term

$$
\left(\frac{1}{r} \frac{\partial \psi}{\partial r}\right)
$$

konstant sein muß. Da die Temperatur axialsymmetrisch verläuft, ist als Temperaturrandbedingung $\partial T / \partial r=0$ gewählt worden.

\subsubsection{Temperaturanfangsbedingung}

Als Temperaturanfangsbedingung $T(t=0)$ sind für stationäre Rechnungen (d.h. mit einem zeitlich konstantem Volumenfluß $Q$ ) meistens Temperaturfelder verwendet worden, bei denen das Temperaturprofil an der Unterseite in vertikaler Richtung etwas ,ausgeschmiert“ worden ist, um numerische Instabilitäten durch zu scharfe Temperaturkontraste zu vermeiden. In den verbleibenden Bereichen ist die Temperatur auf Null (色Referenztemperatur) gesetzt worden. Bei Rechnungen mit zeitlich variablem Injektionsvolumenfluß sind Temperaturfelder von stationären Plumekanälen als Anfangsbedingung verwendet worden.

\subsection{Diskretisierung und Lösungsverfahren}

Zur Lösung des aus Wärmetransportgleichung und Stokes-Gleichung bestehenden Differentialgleichungssystems wird ein Finite-Differenzen-Verfahren verwendet, bei dem die physi- 
kalischen Größen an diskreten Gitterpunkten im Zylinder bestimmt werden. Dazu werden in dieser Modellierung nichtäquidistante Gitterabstände in radialer und vertikaler Richtung verwendet. Das hat insbesondere in radialer Richtung den Vorteil, daß das Plumezentrum mit seinen großen Geschwindigkeits- und Temperaturkontrasten numerisch besser aufgelöst werden kann als die äußeren Regionen, in denen die physikalischen Größen nicht stark variieren.

Als Grundlage für die komplette Diskretisierung der verwendeten Gleichungen dienen die zentralen Differenzenformeln für die erste und die zweite Ableitung bei nichtäquidistanten Gitterabständen, die hier exemplarisch als radiale Ableitungen von $\psi$ formuliert sind:

$$
\begin{aligned}
\left(\frac{\partial \psi}{\partial r}\right)_{i, j} & =\frac{\psi_{i+1, j}}{2 h_{+}}+\psi_{i, j}\left(\frac{1}{2 h_{-}}-\frac{1}{2 h_{+}}\right)-\frac{\psi_{i-1, j}}{2 h_{-}} \\
\left(\frac{\partial^{2} \psi}{\partial r^{2}}\right)_{i, j} & =\frac{2}{h_{+}\left(h_{+}+h_{-}\right)} \psi_{i+1, j}-\frac{2}{h_{+} h_{-}} \psi_{i, j}+\frac{2}{h_{-}\left(h_{+}+h_{-}\right)} \psi_{i-1, j}
\end{aligned}
$$

Dabei ist $h_{-}$der linksseitige und $h_{+}$der rechtsseitige Abstand zwischen dem Zentrumspunkt und den nächsten Nachbarpunkten. Die Ableitungen in vertikaler Richtung ergeben sich in analoger Form. Die gemischten Ableitungen und die Ausdrücke höherer Ordnung sind durch Ineinandereinsetzen der obigen Ausdrücke abgeleitet worden. So ergibt sich zum Beispiel für die gemischte Ableitung in radialer und vertikaler Richtung von $\psi$ der folgende Ausdruck

$$
\left(\frac{\partial^{2} \psi}{\partial r \partial z}\right)_{i, j}=\frac{\partial}{\partial z}\left(\frac{\partial \psi}{\partial r}\right)_{i, j}=\frac{1}{2 k_{+}}\left(\frac{\partial \psi}{\partial r}\right)_{i, j+1}+\left(\frac{\partial \psi}{\partial r}\right)_{i, j}\left(\frac{1}{2 k_{-}}-\frac{1}{2 k_{+}}\right)-\frac{1}{2 k_{-}}\left(\frac{\partial \psi}{\partial r}\right)_{i, j-1}
$$

mit $k_{-}$als unterseitigem und $k_{+}$als oberseitigem Abstand zum Zentralpunkt.

Grundsätzlich liegen die verwendeten Differenzenformeln in der Fehlerordnung schlechter als die entsprechenden äquidistanten Ausdrücke, sind aber dennoch für diese Modellierung gewählt worden, da sie bei geschickter Gitterwahl (vergl. Kapitel 3.3.3) mehr Vorteile als Nachteile haben.

\subsubsection{Stokes-Gleichung}

Das Gleichungssystem, das die Stokes-Gleichung A.3 an jeder Stelle des Definitionsgebietes löst, lautet in Matrixschreibweise

$$
\underline{\underline{A}} \cdot \underline{\Psi}=R a \underline{\partial T} \underline{\partial r}
$$

wobei in $\psi$ die Lösungen der Stromfunktionen in Form eines eindimensionalen Vektors zusammengefaßt sind. Die rechte Seite der Gleichung enthält bekannte Einträge, die aus den radialen Ableitungen der Temperaturen multipliziert mit der Ra-Zahl bestimmt werden. Die Einträge der Matrix $\underline{\underline{A}}$ ergeben sich aus der in Differenzenformeln formulierten StokesGleichung, auf deren Darstellung an dieser Stelle verzichtet wird. Dabei treten gemischte 


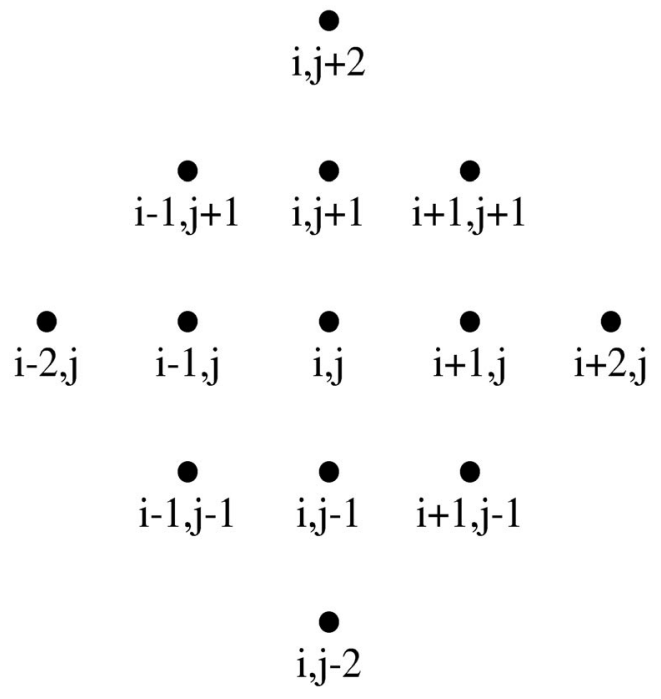

Abbildung 3.2: Differenzenschema, das durch die Diskretisierung der Stokes-Gleichung entsteht. Es gibt an, welche Nachbargitterpunkte zusätzlich zu dem zentralen Punkt $(\mathrm{i}, \mathrm{j})$ nichtverschwindene Einträge in der Matrix $\underline{\underline{A}}$ erzeugen.

Ableitungen und solche bis zur vierten Ordnung auf, so daß sich der in Abbildung 3.2 dargestellte „Differenzenstern“ ergibt. Dieses Schema gibt an, welche Nachbargitterpunkte von dem jeweils zentralen Punkt (i,j) in der Matrix $\underline{\underline{A}}$ Einträge verursachen. Dadurch entsteht eine Bandmatrix mit der in Abbildung 3.3 dargestellten Form. Sie hat eine Dimension von

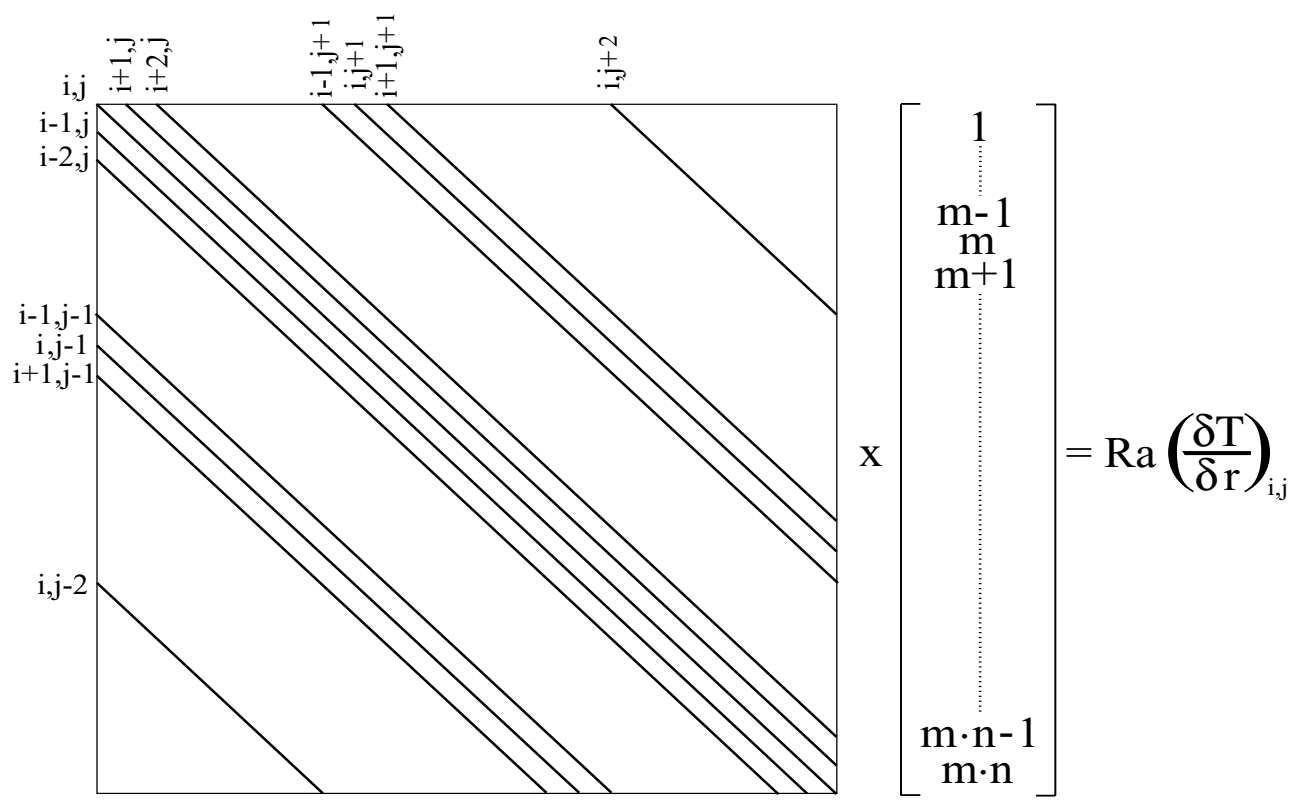

Abbildung 3.3: Besetzung der durch das Differenzenschema entstehenden Bandmatrix. Für die Darstellung sind $m$ Punkte in radialer (i-) Richtung und $n$ Punkte in vertikaler (j-) Richtung mit $\mathrm{m}<\mathrm{n}$ angenommen worden. Die Bezeichnungen der Diagonalen an der Matrix erklären den Zusammenhang mit dem in Abbildung 3.2 dargestellt Differenzenschema. 
$(m \cdot n)^{2}$, wobei $m$ die Anzahl der Gitterpunkte in radialer (i-) Richtung und $n$ die Anzahl der Gitterpunkte in vertikaler (j-) Richtung ist. Die Wahl der Indizes bzw. die Durchnumerierung des Rechengebietes erfolgt zuerst in der Richtung, die weniger Gitterpunkte aufweist. Für die Darstellung 3.3 ist angenommen worden, daß $m<n$. Beim Lösen des Gleichungssystems füllen sich die Bereiche zwischen den Bändern auf. Durch kompaktes Abspeichern (nach Press et al., [1992]) reduziert sich die Matrixdimension jedoch auf $4 m^{2} n$ (wenn $m<n$ ). Da es für diese Art von Bandmatrix keine speziellen Lösungsverfahren gibt, wird hier auf ein Gauß-Verfahren als Lösungsalgorithmus zurückgegriffen.

Um die in Kapitel 3.2 beschriebenen Randbedingungen erfüllen zu können, müssen zusätzlich zu den Matrixelementen des Rechengebietes zum Teil noch Anteile von bis zu zwei virtuellen Gitterebenen berücksichtigt werden. Diese sind dann entweder zu den normalen Elementen der Matrix hinzuzuaddieren oder aber auf die rechte Seite der Gleichung zu kopieren, wenn es sich zum Beispiel bei vorgegebener Stromfunktion um bekannte Werte handelt.

\subsubsection{Wärmetransportgleichung}

Zum Lösen der Wärmetransportgleichung 3.28 wird ein sogenanntes ADI- (Alternative Direction Implicit) Verfahren verwendet. Bei der Methode handelt es sich um ein teilimplizites Verfahren, bei dem jeder Zeitschritt aus zwei Einzelschritten besteht. Zunächst wird ein Teilschritt von der Zeitebene $k$ auf die Zwischenzeitebene $k+1 / 2$ und anschließend ein zweiter von der Zwischenebene $k+1 / 2$ auf die Zeitebene $k+1$ durchgeführt. Dabei werden im ersten Teilschritt beispielsweise zuerst die radialen Komponenten des Advektions- und Diffusionsterms in der neuen (Zwischen-)Zeitebene $k+1 / 2$, d.h. implizit und die vertikalen Komponenten in der alten Zeitebene $k$, also explizit formuliert. Im zweiten Teilschritt werden dann umgekehrt die vertikalen Komponenten in der neuen Zeitebene $k+1$ implizit und die radialen Komponenten in $k+1 / 2$ explizit genommen. Das entsprechende Differenzenschema ist in Abbildung 3.4 dargestellt, und die vollständige exemplarische Diskretisierung des ersten Teilschritts für die Wärmetransportgleichung 3.28 findet sich im Anhang A.2.2. Die hier verwendeten Differenzenformeln für die Zeitableitung lauten

$$
2 \frac{T_{i j}^{k+1 / 2}-T_{i j}^{k}}{\Delta t} \quad \text { bzw. } \quad 2 \frac{T_{i j}^{k+1}-T_{i j}^{k+1 / 2}}{\Delta t}
$$

Das ADI-Verfahren hat den Vorteil, daß es im Vergleich zu expliziten Verfahren für alle Zeitschrittlängen stabil ist und im Vergleich zu anderen impliziten Verfahren nur tridiagonale Gleichungssysteme gelöst werden müssen, da im impliziten Anteil jeweils nur Temperaturen entlang einer Gitterpunktzeile oder Spalte miteinander durch die Differenzenformeln verknüpft werden.

Grundsätzlich ist das Verfahren von zweiter Konvergenzordnung in Raum und Zeit. Da hier jedoch immer nur die Geschwindigkeiten aus der alten Zeitebene verwendet werden, wird diese Fehlerordnung in diesem Fall nicht erreicht. Die räumliche Fehlerordnung hängt auch hier von der Wahl des nichtäquidistanten Gitters ab (vergl. Kapitel 3.3.3). 


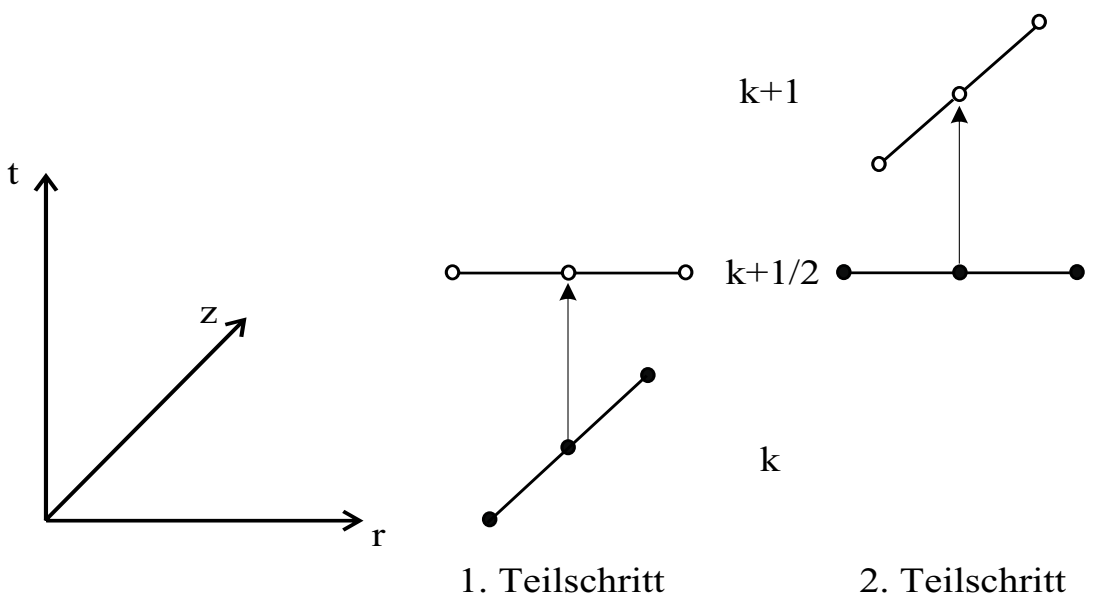

Abbildung 3.4: Schema des ADI-Verfahrens. Die ausgefüllten Kreise symbolisieren die Gitterpunkte, die für die explizit genommenen Ableitungen benötigt werden. Die offenen Kreise stehen für die Gitterpunkte der implizit formulierten Ableitungen.

Die Zeitschrittlänge wird bei zeitabhängigen Rechnungen nach dem Courant-Kriterium gewählt. Anschaulich gibt das Kriterium an, daß die Advektion während eines Zeitschritts das Material um höchstens eine Gitterebene weitertransportieren darf. Für die Raumrichtungen $r$ und $z$ lautet es

$$
\max _{i, j}\left(\left|\frac{u_{i, j} \Delta t}{h_{i, j}}\right|,\left|\frac{w_{i, j} \Delta t}{k_{i, j}}\right|\right) \leq 1,
$$

wobei für die Gitterabstände $h_{i, j}$ und $k_{i, j}$ jeweils das Mittel zwischen den Einzelabständen $h_{+}$und $h_{-}$bzw. $k_{+}$und $k_{-}$gewählt wird. Bei den meisten hier durchgeführten zeitabhängigen Rechnungen ist die Zeitschrittlänge so gewählt worden, daß das Courant-Kriterium mit einem Wert von 0.9 erfüllt ist. Für Rechnungen, bei denen lediglich eine stationäre Lösung bestimmt werden soll, kann das Courant-Kriterium deutlich überschritten werden, da das ADI-Verfahren auch für größere Zeitschritte stabil arbeitet. Dabei dürfen die Advektionslängen, die während eines Zeitschritts auftreten, maximal so groß wie die charakteristischen Modellausdehnungen sein.

Als zusätzliche Option bietet die numerische Modellierung die Möglichkeit, die Rechnungen mit einem sogenannten „Upwind“-Verfahren durchzuführen. Dieses Verfahren verhindert numerische Oszillationen, die in schlecht aufgelösten thermischen Grenzschichten ihren Ursprung haben und sich über das gesamte Rechengebiet ausbreiten können. Dafür werden in Abhängigkeit der als

$$
P e_{r i, j}=\frac{u_{i, j} \cdot h}{\kappa} \quad \text { bzw. } P e_{z i, j}=\frac{w_{i, j} \cdot k}{\kappa}
$$

definierten Gitter-Peclet-Zahlen an der Stelle $(i, j)$ für die radiale und die vertikale Richtung die Differenzenquotienten des Advektionsterms einseitig gegen die Strömungsrichtung stär- 
ker gewichtet. Die mit dem Gewichtungsfaktor $\gamma$ modifizierten Differenzenformeln lauten

$$
\begin{aligned}
& \left(\frac{\partial T}{\partial r}\right)_{i, j} \simeq \frac{\left(1-\gamma_{r}\right) T_{i+1, j}}{2 h_{+}}+\left(\frac{1+\gamma_{r}}{2 h_{-}}-\frac{1-\gamma_{r}}{2 h_{+}}\right) T_{i, j}-\frac{\left(1+\gamma_{r}\right) T_{i-1, j}}{2 h_{-}} \\
& \left(\frac{\partial T}{\partial z}\right)_{i, j} \simeq \frac{\left(1-\gamma_{z}\right) T_{i, j+1}}{2 k_{+}}+\left(\frac{1+\gamma_{z}}{2 k_{-}}-\frac{1-\gamma_{z}}{2 k_{+}}\right) T_{i, j}-\frac{\left(1+\gamma_{z}\right) T_{i, j-1}}{2 k_{-}}
\end{aligned}
$$

wobei

$$
\gamma_{r}=\frac{P e_{r i, j}}{\left(4^{2}+P e_{r i, j}^{2}\right)^{1 / 2}} \quad \text { bzw. } \quad \gamma_{z}=\frac{P e_{z i, j}}{\left(4^{2}+P e_{z i, j}^{2}\right)^{1 / 2}},
$$

so daß für verschwindende $P e$-Zahlen die Differenzenquotienten unverändert bleiben bzw. für vom Betrag her kleine $P e$-Zahlen eine einseitige Übergewichtung der Differenzenformeln stattfindet. Für $P e \gg 2$ bzw. $P e \ll-2$ hingegen, gilt $\gamma \rightarrow+1$ bzw. $\gamma \rightarrow-1$, so daß die Differenzenformeln 3.41 bzw. 3.42 zu einseitigen werden. Durch die Verwendung des UpwindVerfahrens wird dem System eine künstliche anisotrope numerische Diffusion in Richtung der Strömung aufgeprägt, die mögliche Oszillationen unterdrückt. Da diese Diffusion jedoch nur an den Punkten mit erhöhter Advektionsgeschwindigkeit auftritt, ist sie für den Gesamtwärmetransport zu vernachlässigen. Bei dem hier verwendeten Upwind-Verfahren gehen für den theoretischen Fall immer kleiner werdender Gitterabstände $h \rightarrow 0$ bzw. $k \rightarrow 0$ die Differenzenausdrücke in die nicht modifizierten Ausdücke über, so daß sich die Konvergenzordnung genau genommen nicht ändert.

\subsubsection{Gitter}

Das Programm arbeitet grundsätzlich mit nichtäquidistanten Gittern in radialer und vertikaler Richtung. Die Option auf nichtäquidistante Gitterabstände in vertikaler Richtung ist jedoch bei den meisten Rechnungen nicht genutzt worden, da es bei der Problemstellung mit offenem Unter- und Oberrand keine Bereiche mit ausgesprochen starken vertikalen Gradienten in den physikalischen Größen (z.B. thermische Grenzschichten) gibt. Für Modelle mit geschlossener Ober- und Unterseite, die von unten geheizt und oben gekühlt werden, kann die Verwendung dieser Option jedoch sehr nützlich sein. Das Gitter in radialer Richtung ist so gewählt, daß es im Plumezentrum zunächst auch äquidistant verläuft und erst weiter außen langsam die Gitterabstände vergrößert werden. Das hat den Vorteil, daß für den äquidistanten Bereich die Differenzenformeln (3.34, 3.35) von zweiter Fehlerordnung sind, weil sie dann identisch mit den äquidistanten Ausdrücken sind. Für den nichtäquidistanten Bereich wird die gleiche Fehlerordnung erreicht, wenn sich bei zunehmender Gitterverfeinerung die Gitterabstände $h_{+}$bzw. $k_{+}$und $h_{-}$bzw. $k_{-}$zunehmend ähnlicher werden.

Für den nichtäquidistanten Bereich wird hier eine quadratische Zunahme der Gitterabstände mit dem Radius gewählt. Abbildung 3.5 zeigt ein Beispiel für ein solches Gitter in radialer Richtung.

Um zu entscheiden, wieviele Gitterpunkte in radialer und vertikaler Richtung nötig sind, um eine ausreichend gute numerische Auflösung zu bekommen, sind Vergleichsrechnungen 


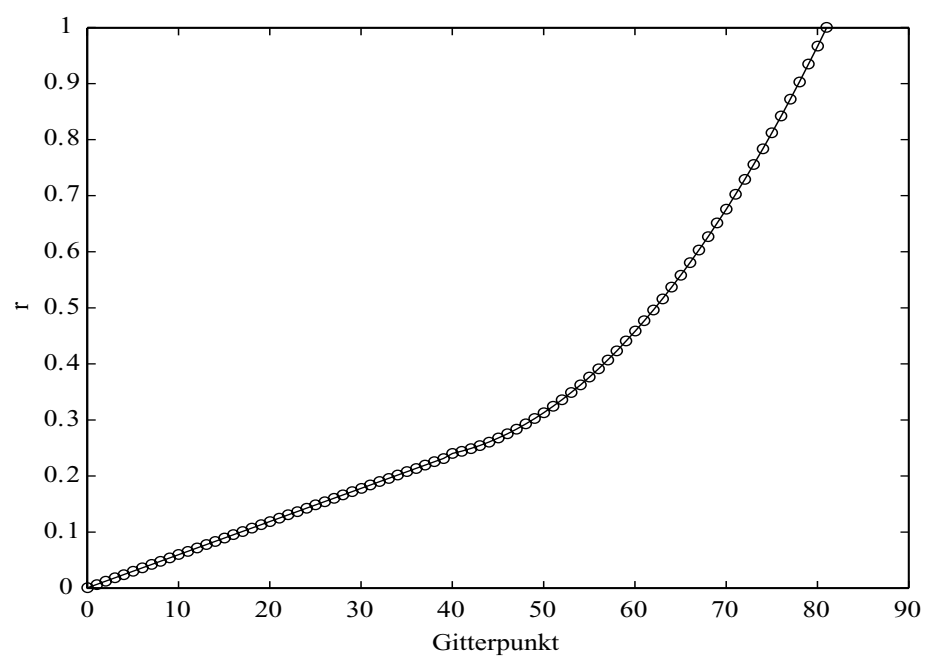

Abbildung 3.5: Gitter in radialer Richtung. Aufgetragen sind die Gitterpunkte gegenüber dem radialen Abstand von Plumezentrum $r$.

mit unterschiedlichen Gittern durchgeführt worden. Abbildung 3.6 zeigt ausgewählte Profile aus den stationären Temperaturfeldern von einigen Rechnungen. Dabei ist eine typische Plumeströmung mit sehr großem Volumenstrom mit unterschiedlichen Gittertypen, ansonsten aber gleichen Parametern berechnet worden. Grundsätzlich zeigen sich keine gravierenden Unterschiede in den Profilen in halber Zylinderhöhe, obwohl mit sehr unterschiedlichen An-

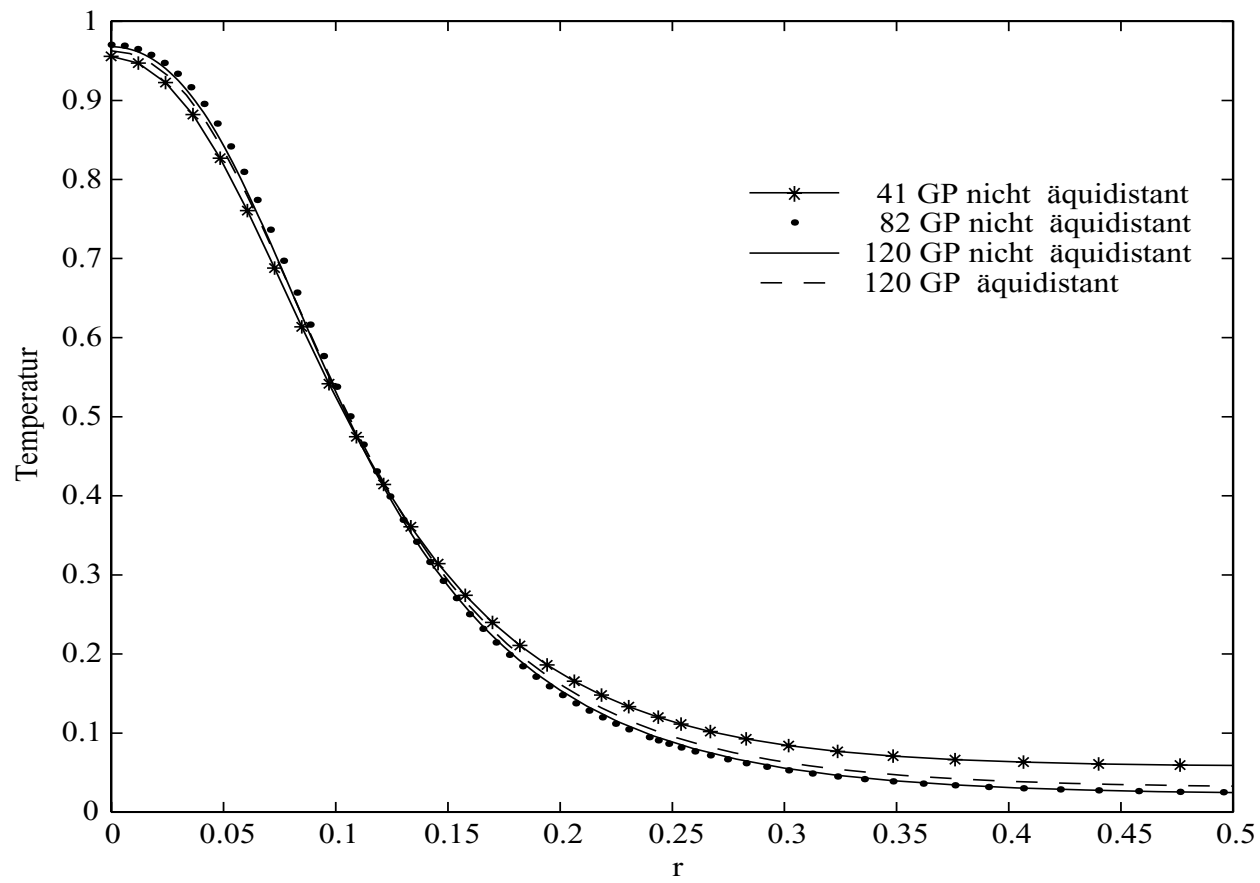

Abbildung 3.6: Temperaturprofile in halber Zylinderhöhe für Vergleichsrechnungen mit unterschiedlichen Gittertypen. 
zahlen von Gitterpunkten (GP) gerechnet worden ist. Betrachtet man den Fall des nichtäquidistanten Gitters mit 120 Punkten als Referenz, da es in dem in Abbildung 3.6 dargestellten Bereich die meisten Gitterpunkte aufweist, so weicht als einziger der Fall mit 41 Gitterpunkten von der Referenzlösung nennenswert ab. Das Gitter mit 82 Gitterpunkten hingegen stimmt sehr gut mit der Referenzlösung überein, so daß es für die meisten Rechnungen als Kompromiß zwischen Rechengeschwindigkeit und den Anforderungen an die Genauigkeit verwendet worden ist.

In vertikaler Richtung sind 160 äquidistant angeordnete Gitterpunkte für eine Zylinderhöhe von 10 bzw. 320 Punkte für eine Zylinderhöhe von 20 Zylinderradien verwendet worden.

\subsection{Programmtests}

Zur Kontrolle der kompletten numerischen Umsetzung existiert kein analytischer Testfall, so daß neben umfangreichen Kontrollen während der Programmierung auch viele Tests der Einzelprogrammkomponenten durchgeführt worden sind. Die Umsetzung der Stokes-Gleichung in Differenzenformeln und die Programmierung des Lösungsverfahrens kann zum Beispiel mit einer analytisch integrierbaren Stromfunktionen für den isoviskosen Fall überprüft werden. Dazu ist die Stromfunktion $\psi_{\text {Test }}(r, z)$ analytisch nach $r$ integriert worden, woraus sich bis auf eine Konstante das Temperaturfeld ergibt. Dann ist unter Verwendung dieses Feldes für die rechte Seite der Gleichung 3.37 der Lösungsalgorithmus gestartet worden, so daß der Stromfunktionsergebnisvektor $\underline{\psi}$ mit der Ausgangsstromfunktion $\psi_{\text {Test }}(r, z)$ verglichen werden konnte. In Abbildung 3.7 sind zwei exemplarische Stromfunktionsprofile dieses Tests dargestellt. Dabei sind die analytischen Stromfunktionen als durchgezogene Linien und die numerisch bestimmten gesternt dargestellt.
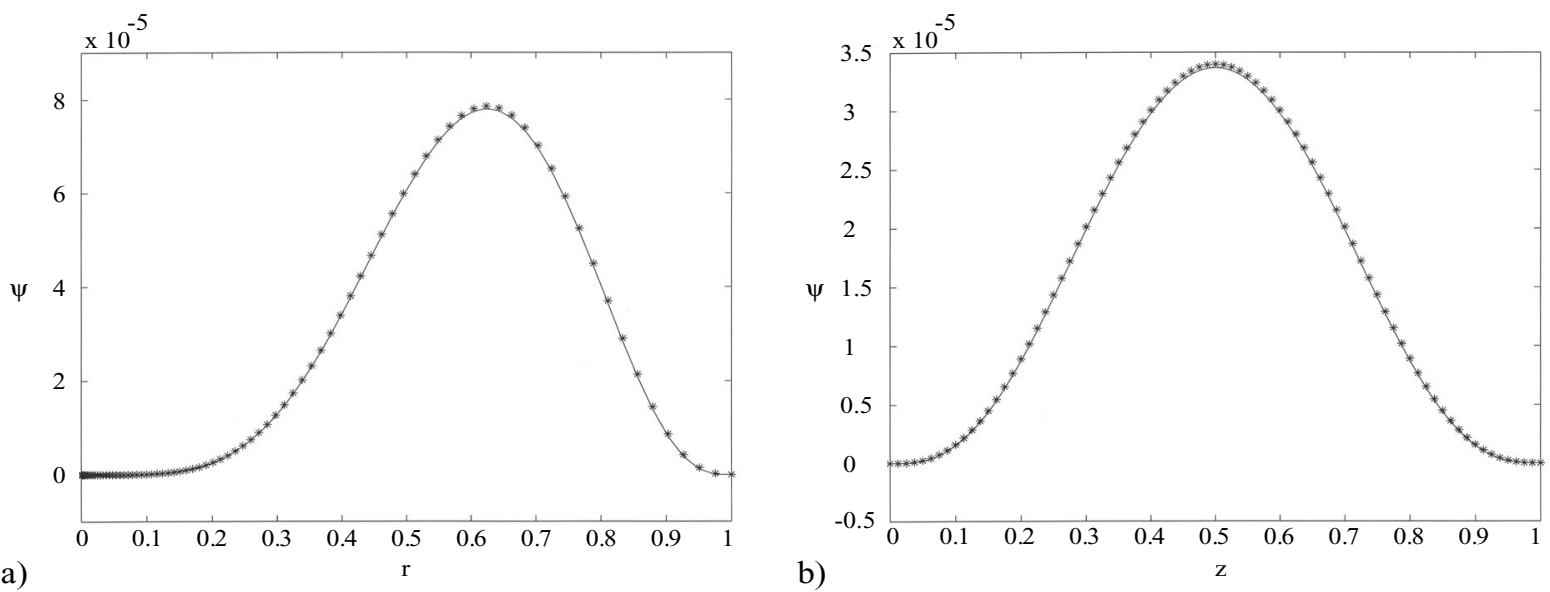

Abbildung 3.7: Vergleich zwischen analytisch (durchgezogen) und numerisch bestimmter Stromfunktion (gesternt) in radialer Richtung bei $z=0.5$ (a) und in vertikaler Richtung bei $r=0.4$ (b). 
Für die Überprüfung der numerischen Umsetzung der Wärmetransportgleichung sind Vergleichsrechnungen mit dem in Albers [2000] vorgestellten Programm „CMG“ durchgeführt worden. Für den Vergleich sind bei offenen Ober- und Unterrändern sowohl die radialen als auch die vertikalen Geschwindigkeitsfelder und ein radialer gaußförmiger Temperaturverlauf an der Unterseite vorgegeben worden. Da das Programm CMG im Gegensatz zu dem hier entwickelten Programm „CFDC“ in einem kartesischen Koordinatensystem arbeitet, sind die Eingabegeschwindigkeitsfelder und der Anfangstemperaturverlauf entsprechend transformiert worden. Abbildung 3.8 zeigt als Ergebnis dieses Tests den zeitlichen Verlauf der Temperaturen $T$ bei $r=0$ in halber Höhe $z=0.5$. Man erkennt für den gesamten Zeitverlauf eine fast perfekte Übereinstimmung zwischen den Ergebnissen. Die geringfügigen systematischen Abweichungen sind auf unterschiedliche Upwind-Verfahren zurückzuführen, weshalb in Abbildung 3.9 auch die vertikalen stationären Temperaturverläufe bei $r=0$ für verschiedene Upwind-Verfahren von CMG dargestellt sind. Das für CFDC verwendete Upwind-Verfahren (vergl. Kapitel 3.3.2) ist mit der dritten Upwind-Variante von CMG (Upw3) vergleichbar, wofür sich auch die beste Übereinstimmung in dem in Abbildung 3.9 dargestellten vertikalen Temperaturverlauf ergibt. Auch für den radialen Verlauf der stationären Temperaturen in halber Zylinderhöhe (Abbildung 3.10) findet sich eine perfekte Übereinstimmung.

Zusammenfassend läßt sich feststellen, daß die hier aufgeführten Tests - gemeinsam mit

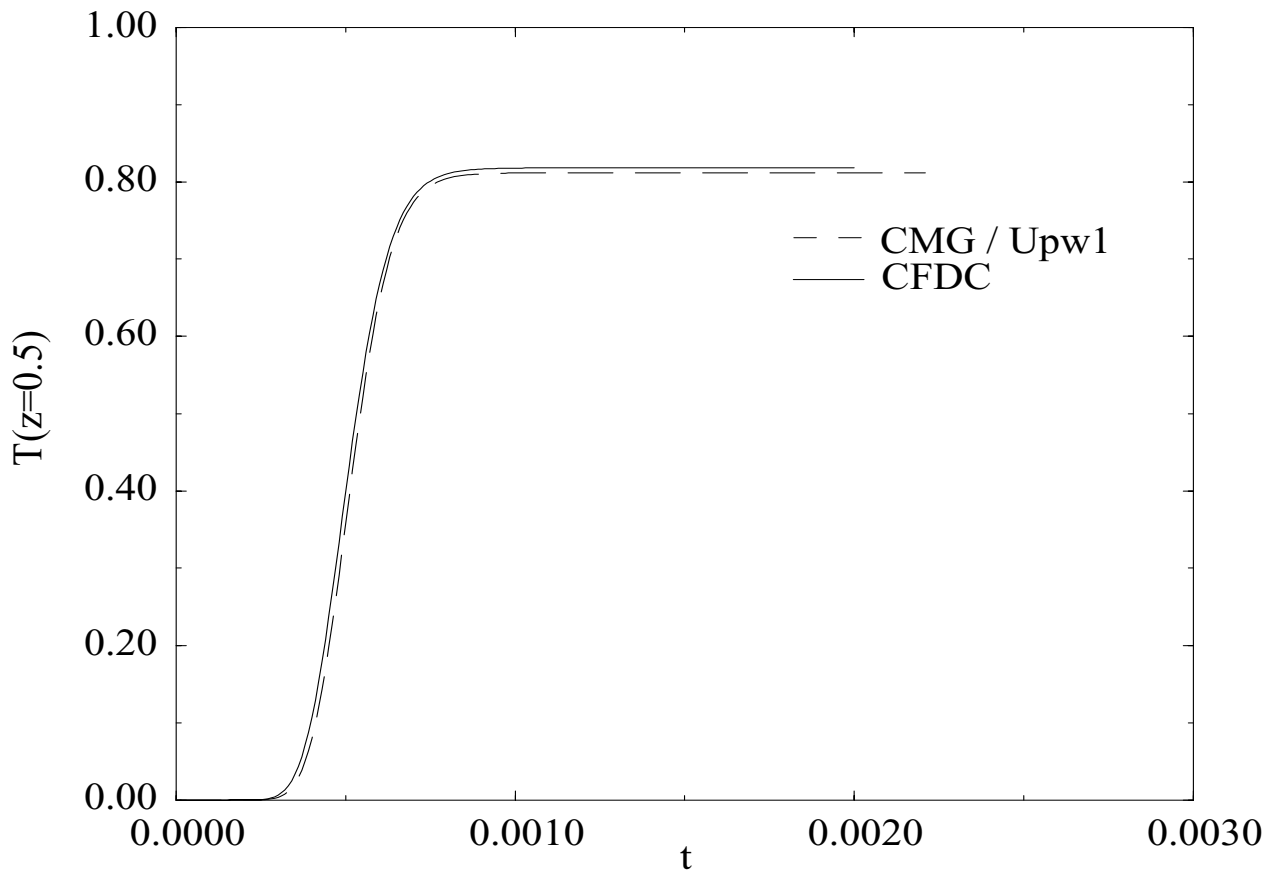

Abbildung 3.8: Vergleich der Zeitentwicklung zwischen CMG und dem hier entwickelten Programm CFDC. Die Darstellung zeigt den zeitlichen Verlauf der Temperatur $T(r=0, z=0.5)$. 


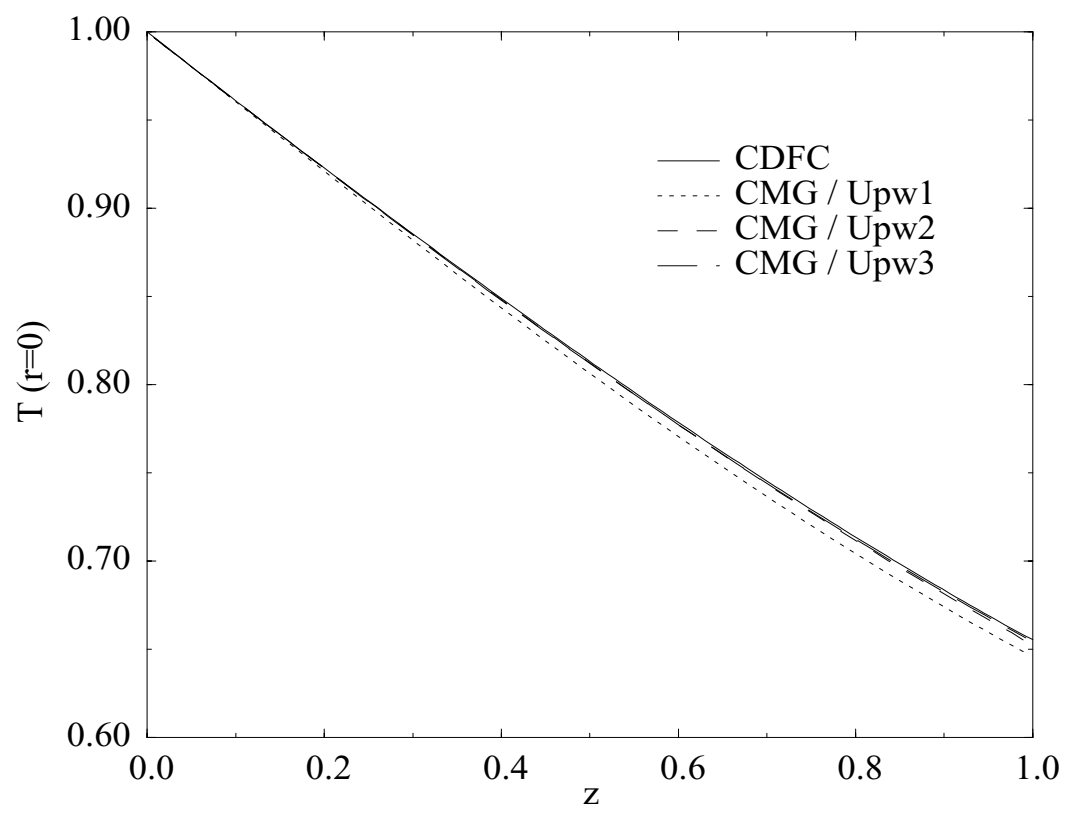

Abbildung 3.9: Vergleich von stationären vertikalen Temperaturprofilen zwischen CMG und CFDC bei $r=0$.

den vielen nicht dargestellten Einzeltests zum Aufstellen und Lösen der Matrixgleichungen und weitere Vergleichsrechnungen mit dem Programm CMG (auch für temperaturabhängige Viskosität) - zeigen, daß das hier entwickelte Programm CFDC korrekt arbeitet und daher für die Modellierung von thermischen Plumes verwendet werden kann.

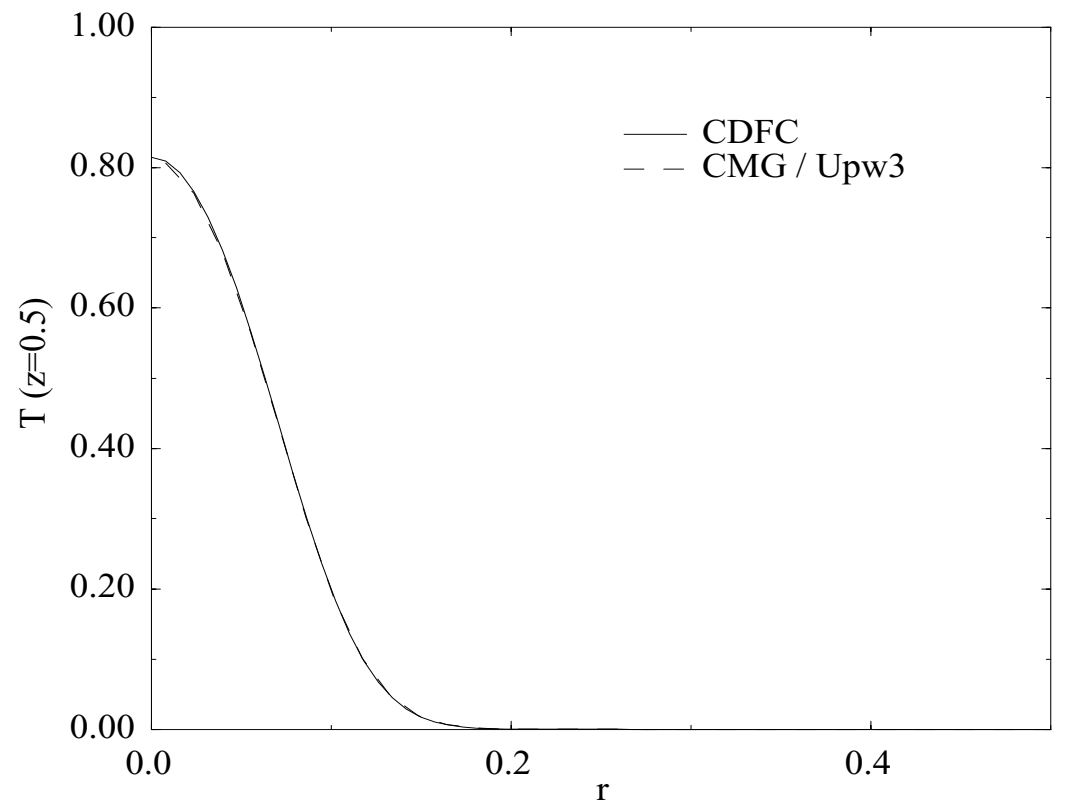

Abbildung 3.10: Vergleich von stationären radialen Temperaturprofilen zwischen CMG und CFDC bei $z=0.5$. 


\section{Kapitel 4}

\section{Beispielergebnisse und Vergleiche zwischen Numerik und Experiment}

In diesem Kapitel werden Vergleiche zwischen der numerischen und der experimentellen Modellierung angestellt. Durch die in Kapitel 2 vorgestellten Meßmethoden können diese Vergleiche quantitativ erfolgen, was für die unabhängige Kontrolle der verwendeten Meßverfahren sehr nützlich ist. Ein Vergleich ist insbesondere im Hinblick auf die Temperaturmeßmethode von großer Bedeutung, da diese im Gegensatz zur PIV-Methode nur in dieser Arbeit Verwendung findet und daher wenig etabliert ist. Die bei den Vergleichen auftretenden Abweichungen zwischen numerischen und experimentellen Ergebnissen werden in diesem Kapitel diskutiert und auf ihre Ursachen hin untersucht.

Gleichzeitig werden anhand von Beispielergebnissen bestimmte Plume- und Welleneigenschaften verdeutlicht, die für ein tieferes Verständnis des physikalischen Systems von Nutzen sind. Im ersten Abschnitt werden zunächst Ergebnisse und Vergleiche für stationäre Plumes dargestellt, bevor dann im zweiten auf die zeitabhängigen Wellenphänomene eingegangen wird.

\subsection{Stationäre Plumes}

\subsubsection{Temperaturen}

Für den im Folgenden dargestellten Vergleich zwischen Experiment und Numerik sind Rechnungen mit konstantem Volumenstrom und dem rein thermischen Viskositätsgesetz 2.1 des Sirups durchgeführt worden. In der numerischen Modellierung steigt analog zum Laborexperiment zunächst auch ein Diapir auf, dem ein stationärer Kanal nachfolgt. Abbildung 4.1 a) zeigt ein exemplarisch herausgegriffenes Temperaturfeld, bei dem der Diapir die Oberseite des Rechengebietes noch nicht erreicht hat. Der Temperaturverlauf im Plumekopf läßt darauf schließen, daß auch hier das im Experiment zu beobachtende spiralartige Einbinden von Umgebungsmaterial auftritt. Erst wenn der Diapir das Rechengebiet durch den obereren offenen Rand verlassen hat und sich die Temperaturen zwischen zwei Zeitschritten nicht 


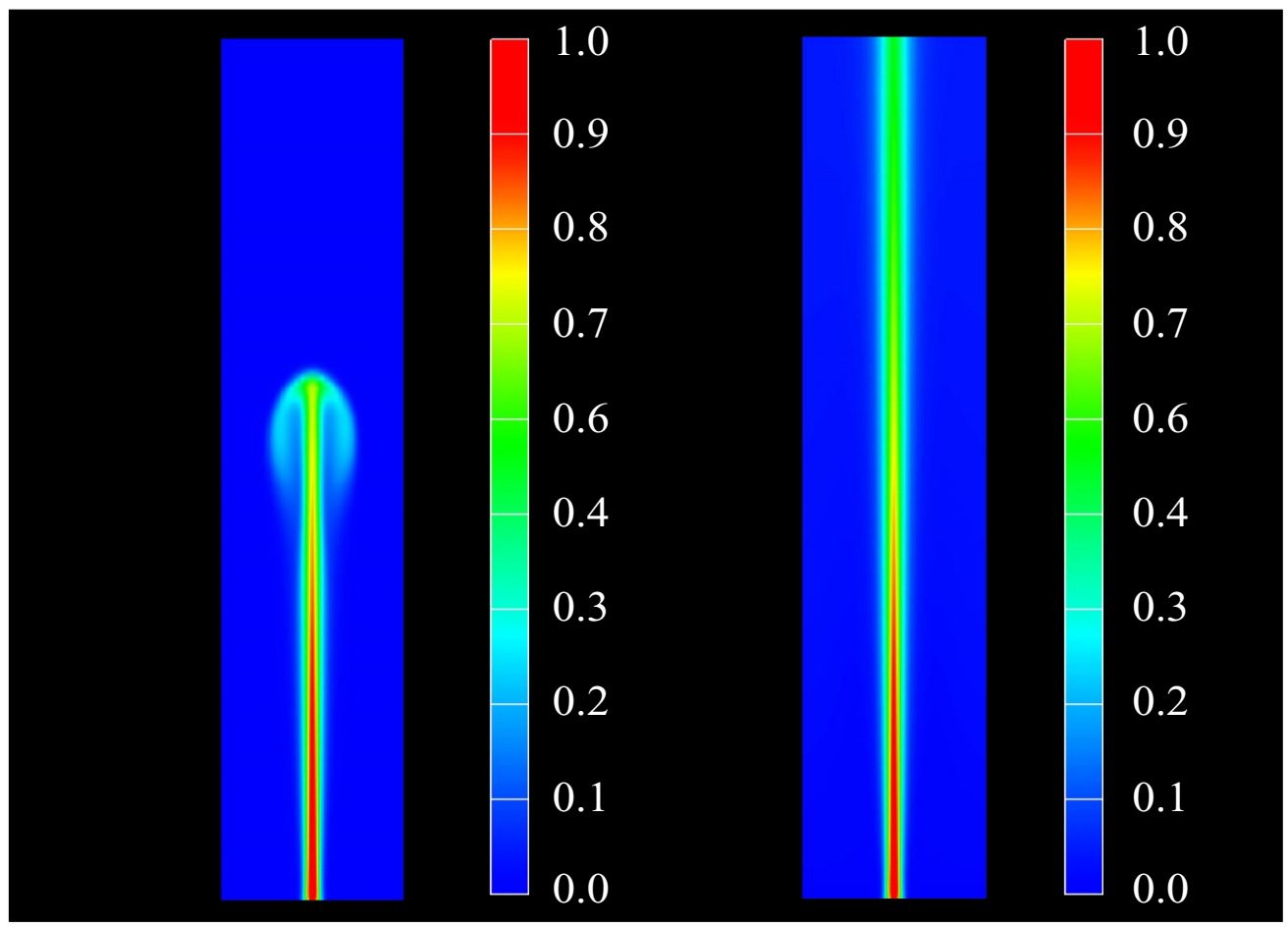

a)

b)

Abbildung 4.1: Temperaturfelder eines numerisch berechneten Plumes. Dargestellt ist ein exemplarisch herausgegriffener Zeitschritt einer Rechnung mit konstantem Volumenstrom (a) und das sich später einstellende stationäre Temperaturfeld (b).

mehr verändern, ist ein stationärer Zustand erreicht mit dem die experimentell bestimmten Temperaturen verglichen werden (Abbildung $4.1 \mathrm{~b}$ ).

In Abbildung 4.2 ist ein derartiger Vergleich für zwei unterschiedliche Volumenflüsse dargestellt. Es werden jeweils radiale Temperaturprofile in unterschiedlichen Aufstiegshöhen miteinander verglichen. Die Temperaturen sind so mit der Temperaturanomalie am Einströmpunkt und der Matrixtemperatur skaliert worden, daß sie zwischen 0 und 1 variieren. Generell beobachtet man eine sehr gute Übereinstimmung zwischen den Ergebnissen. Im Plumezentrum ( $r=0$ ) beträgt die maximale Abweichung lediglich 3-4\%. Die Form des Kurvenverlaufs stimmt ebenfalls gut überein. Lediglich bei dem in Abbildung 4.2 b) dargestellten Fall weichen die Halbwertsbreiten der Kurven um etwa 10\% voneinander ab. In allen anderen Fällen sind die Unterschiede deutlich kleiner. Die beobachteten Abweichungen liegen im Rahmen der in Kapitel 2.2.3 diskutierten Fehlerquellen und sind vermutlich nicht auf voneinander abweichende Bedingungen zwischen der numerischen und der experimentellen Modellierung (z.B. Prandtl-Zahl, Randbedingungen oder Gefäßgeometrie) oder auf unzureichende numerische Auflösung zurückzuführen.

Neben den in Kapitel 2.2.3 diskutierten systematischen Abweichungen könnte eine weitere Ursache für die kleinen Differenzen in der Streuung des Laserstrahls liegen, die im Tank 

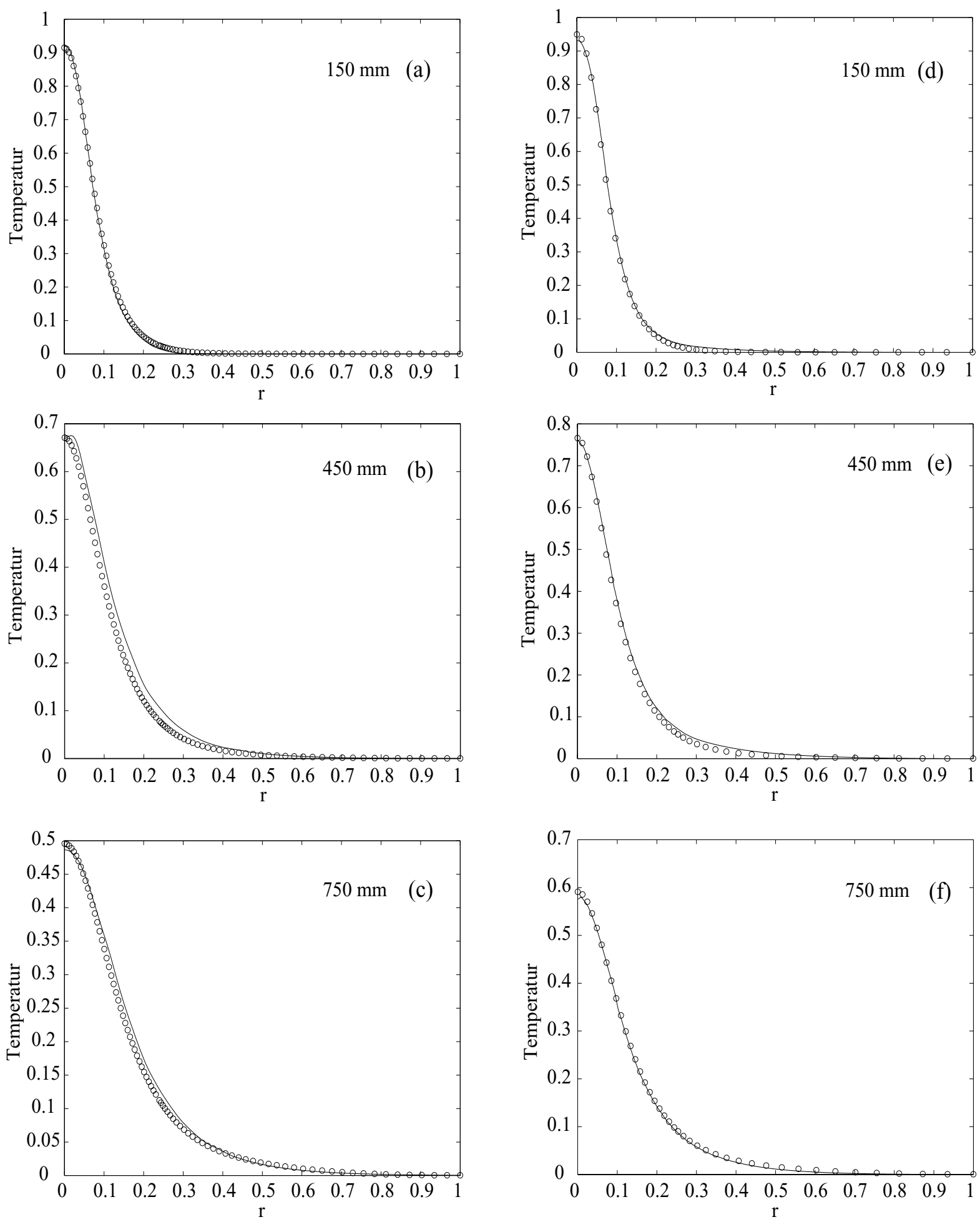

Abbildung 4.2: Vergleich zwischen experimentellen (durchgezogen) und numerisch bestimmten (Kreise) radialen Temperaturprofilen in unterschiedlichen Aufstiegshöhen und für zwei verschiedene Volumenflüsse: (a)-(c) $30 \mathrm{ml} / \mathrm{min}$; (d)-(f) $40 \mathrm{ml} / \mathrm{min}$. 
auftritt und die zu einer Strahlaufweitung führt. Die Aufweitung läßt das in der CCD-Kamera gemessene Intensitätsprofil unregelmäßiger werden, so daß Unsicherheiten bei der Bestimmung des Laserstrahlzentrums entstehen. Dadurch ist den Meßwerten für die Ablenkungswinkel ein statistisches Rauschen überlagert, welches ebenfalls einen Teil der Abweichungen erklären kann, wenn man bedenkt, daß die maximal auftretenden Ablenkungswinkel im Plume lediglich bei ein bis zwei Grad liegen (vergl. Abbildung $2.5 \mathrm{~b}$ ).

Angesichts der kleinen Unterschiede zwischen Experiment und Numerik kann man davon ausgehen, daß die Temperaturmeßmethode sehr genau arbeitet und für die Bestimmung der Kanaltemperaturen gut geeignet ist.

\subsubsection{Geschwindigkeiten}

Zur Geschwindigkeitsbestimmung sind neben aufwendigen PIV-Messungen auch sehr einfache Messungen durch Verfolgung von in die Strömung injizierten Farbpartikeln durchgeführt worden. Dabei ist es wichtig, daß die zusätzlich eingebrachte Farbe die gleiche Dichte wie das Plumematerial hat, damit sie sich in der Strömung passiv verhält. Die Methode ist ausschließlich zur Bestimmung der maximalen vertikalen Geschwindigkeit im Plumezentrum geeignet und kann keine Aussagen über den radialen Verlauf der Vertikalgeschwindigkeiten liefern, da die Farbe in vertikaler Richtung stark ,,ausschmiert“, so daß lediglich die schnellsten Farbpartikel beobachtbar sind.

In Abbildung 4.3 werden die mit dieser Methode bestimmten Geschwindigkeiten in verti-

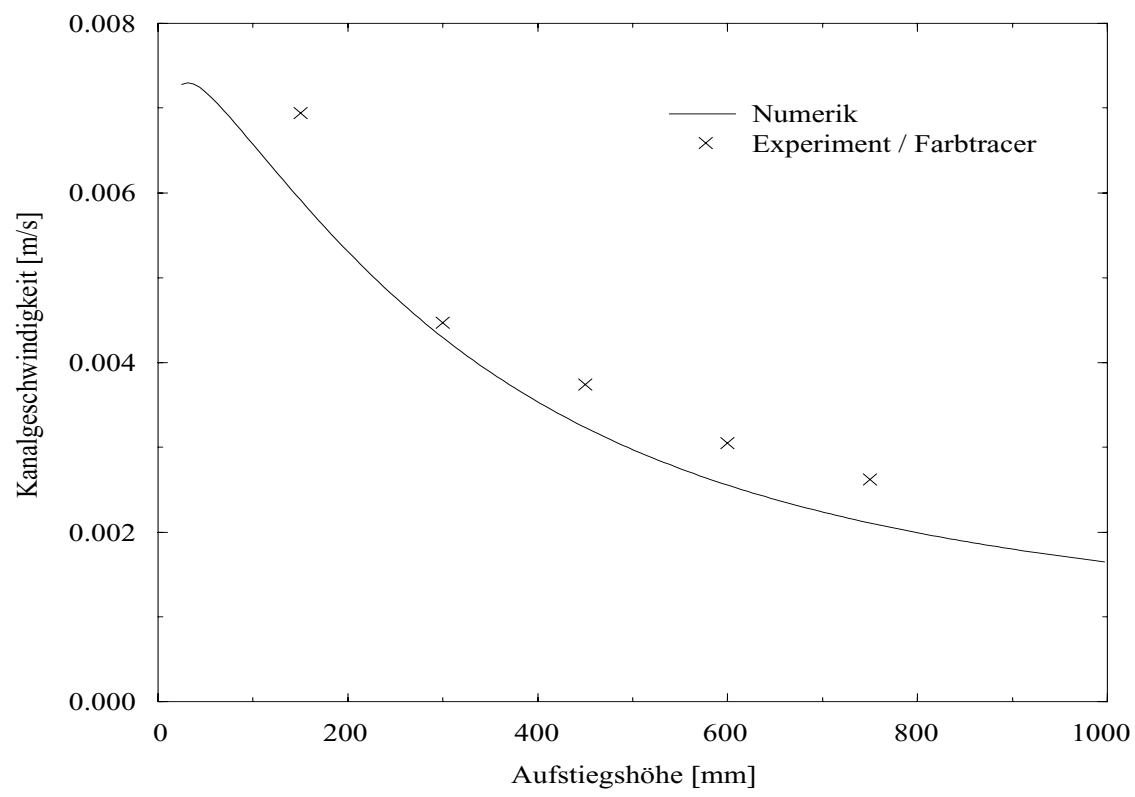

Abbildung 4.3: Vergleich der Maximalgeschwindigkeiten in einem Plumekanal mit einem Volumenfluß von $30 \mathrm{ml} / \mathrm{min}$ und einem Temperaturkontrast von $59 \mathrm{~K}$. Die Geschwindigkeitsmessungen sind mit Hilfe zusätzlich injizierter Farbpartikel durchgeführt worden. 
kaler Richtung mit numerisch berechneten Geschwindigkeiten im Plumezentrum in verschiedenen Aufstiegshöhen verglichen. Man erkennt eine ungefähre Übereinstimmung der Daten mit einer systematischen Abweichung zwischen numerischen und gemessenen Werten von etwa 10-15\%. Die Ursache für die Abweichungen könnte darin liegen, daß das zusätzlich injizierte Material eine geringfügig niedrigere Dichte als das Plumematerial hatte. Außerdem entstehen bei dieser Meßmethode Unsicherheiten durch das Auseinanderdiffundieren der Farbe, die sich so in größeren Aufstiegshöhen zunehmend im Kanal verteilt, was die Beobachtung erschwert und zu Fehlern bei der Zeitmessung führen kann.

Das Beispiel zeigt, daß die Farbpartikelmethode für eine grobe Bestimmung der vertikalen Geschwindigkeiten gut geeignet ist. Sie bringt neben genannten Meßunsicherheiten allerdings den Nachteil mit sich, daß durch das Einbringen der Farbpartikel der Sirup mit der Zeit verunreinigt wird. Aus diesem Grund und zur Bestimmung von vollständigen Geschwindigkeitsfeldern ist im weiteren die in Kapitel 2.3 beschriebene PIV-Methode verwendet worden.

In Abbildung 4.4 a) ist ein mit der PIV-Methode gemessenes vertikales Geschwindigkeitsfeld im Vergleich zu dem entsprechenden numerisch bestimmten Feld (Abbildung 4.4 b) für einen stationären Plumekanal dargestellt. Bis auf die kleinen, nicht herausgefilterten Bereiche in der PIV-Messung mit offensichtlich falsch bestimmten Geschwindigkeiten (Abbildung 4.4 a oben links) erkennt man eine gute Übereinstimmung zwischen Numerik und Ex-
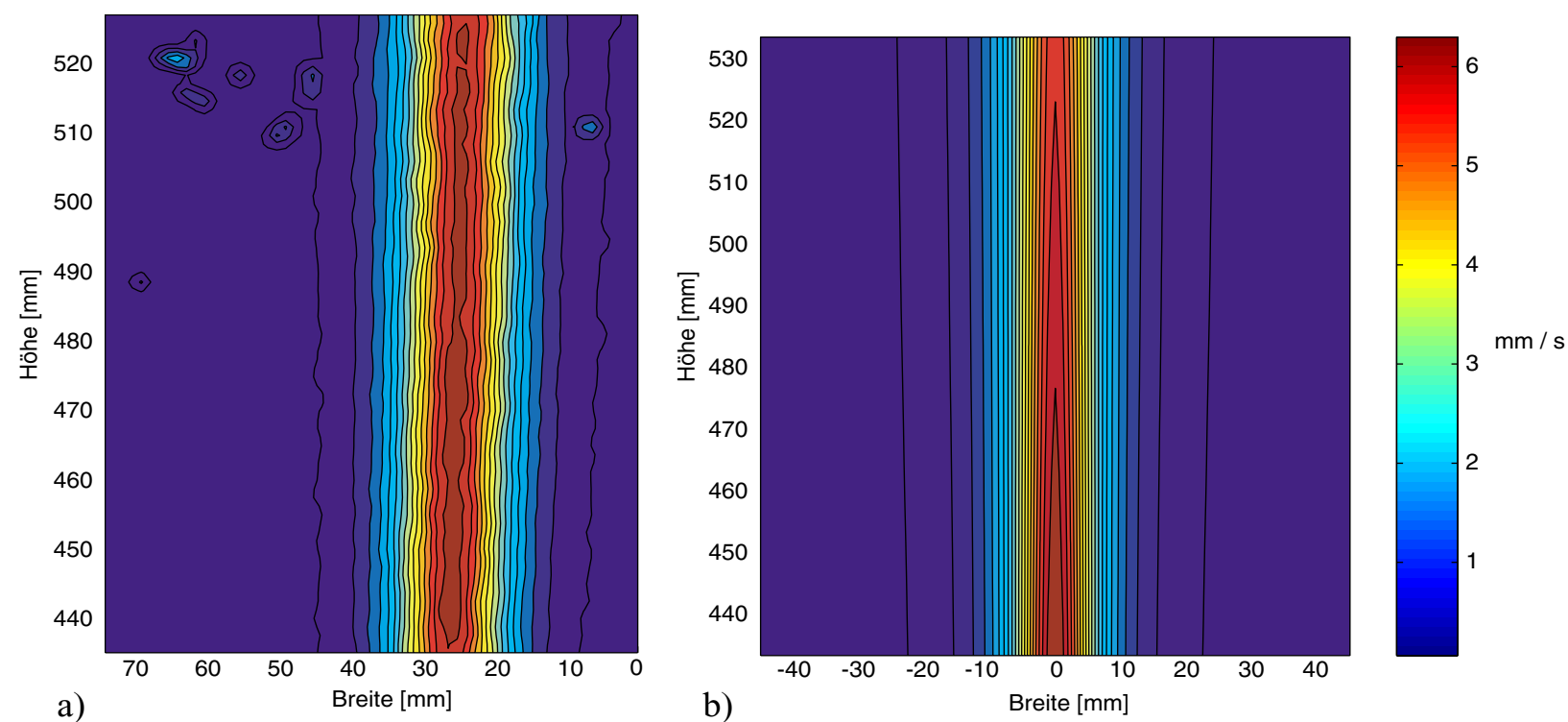

Abbildung 4.4: Vergleich zwischen einem gemessenen vertikalen Geschwindigkeitsfeld (a) und einem numerisch bestimmten (b) für einen stationären Plumekanal mit einem Volumenfluß von $60 \mathrm{ml} / \mathrm{min}$ und einer Temperaturanomalie von $66 \mathrm{~K}$. Für beide Abbildungen wurde die gleiche Farbskala verwendet. 

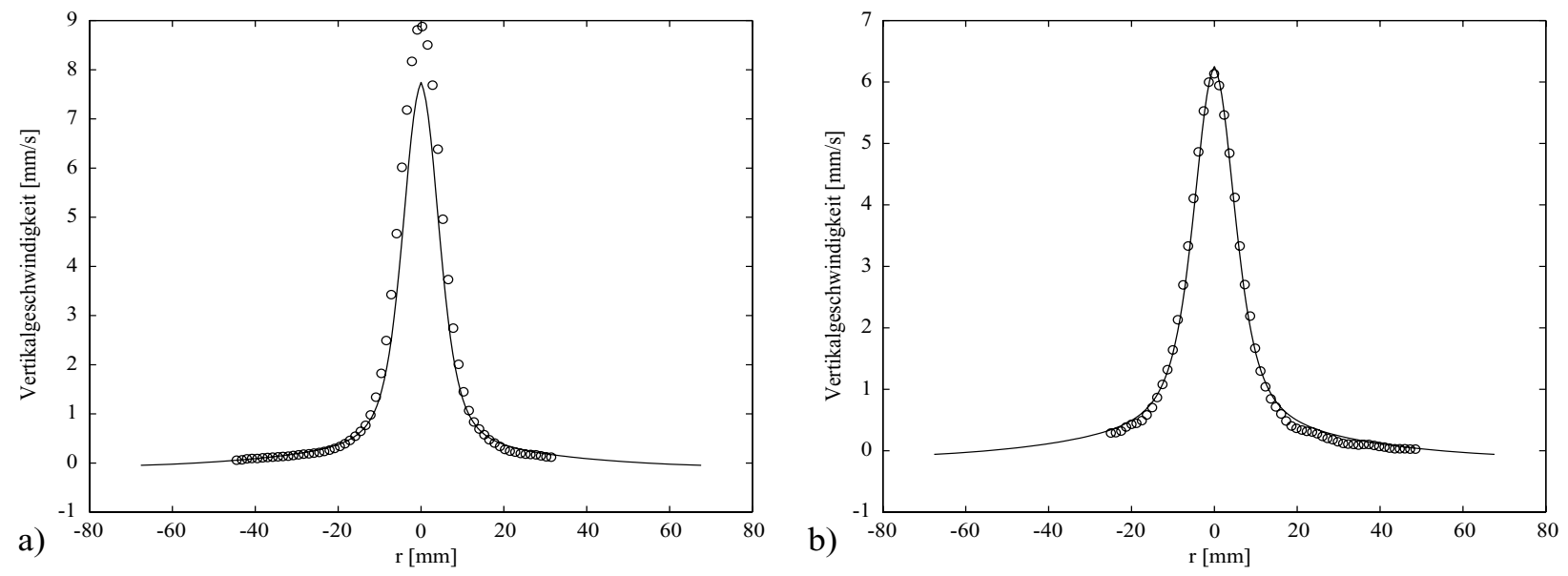

Abbildung 4.5: Vergleich zwischen numerisch (durchgezogen) und experimentell (Kreise) bestimmten vertikalen Geschwindigkeitsprofilen eines stationären Plumekanals mit einem Volumenfluß von $60 \mathrm{ml} / \mathrm{min}$ und einer Temperaturanomalie von $66 \mathrm{~K}$ in einer Aufstiegshöhe von $300 \mathrm{~mm}$ (a) und $480 \mathrm{~mm}$ (b).

periment. Zur Verdeutlichung werden in Abbildung 4.5 Profile der Vertikalgeschwindigkeit für zwei Aufstiegshöhen miteinander verglichen.

Insbesondere für die Messung in $480 \mathrm{~mm}$ Höhe (Abbildung 4.5 b) zeigt sich eine ausgezeichnete Übereinstimmung in Form und Amplitude des Geschwindigkeitsverlaufs. In der niedrigeren Aufstiegshöhe von $300 \mathrm{~mm}$ (Abbildung 4.5 a) liegt die experimentell bestimmte Maximalgeschwindigkeit um 15\% über dem numerischen Wert und die experimentell bestimmte Kurve ist breiter als die numerische. Im Hinblick auf die gute Übereinstimmung in anderen Aufstiegshöhen wird vermutet, daß diese Abweichungen durch Ungenauigkeiten bei der Maßstabsbestimmung der PIV-Bilder entstanden sein könnten. Bei dem hier verwendeten experimentellen Aufbau mit einem nicht fixierten Abstand zwischen CCD-Kamera und Experiment muß nach Veränderung der Meßhöhe oft auch der Fokus der Kamera korrigiert werden, wenn sich der Abstand zum Lichtschnitt dabei geringfügig verändert hat. Da bei dem verwendeten Objektiv mit einer Fokusveränderung auch eine Bildmaßstabsveränderung einhergeht, ist es denkbar, daß die Abweichung auf diesen Effekt zurückgeführt werden kann. Ein aufwendigerer Versuchsaufbau mit einer festmontierten Kamera und einem über Spiegel eingeblendeten Maßstabsgitter, könnte Abhilfe schaffen und die Unsicherheiten bei der Maßstabsbestimmung reduzieren.

\subsection{Solitäre Wellen}

Zur Erzeugung von solitären Wellen ist im Experiment der Volumenfluß des injizierten Materials typischer Weise um den Faktor 5-30 für 1-2 s erhöht worden. In den numerischen Studien ist der untersuchte Parameterbereich auf Faktoren zwischen 2-30 und Zeiten zwi- 
schen 0.5-10 s ausgedehnt worden. In diesem Abschnitt werden für die Vergleiche zwischen Numerik und Experiment und zur Erklärung der Welleneigenschaften typische Beispiele aus diesen Parameterstudien ausgewählt, bevor im nachfolgenden Kapitel 5 die vollständigen Ergebnisse in zusammengefaßter Form aufgeführt werden.

\subsubsection{Temperaturen}

Im Experiment sind zur Untersuchung der Welleneigenschaften Temperaturmessungen in unterschiedlichen Aufstiegshöhen durchgeführt worden. Diese Ergebnisse sind im folgenden meist im Vergleich zu den Temperaturprofilen des ungestörten Plumekanals dargestellt. Auch in diesem Abschnitt wird zunächst der direkte Vergleich zwischen numerischer und experimenteller Modellierung anhand beispielhafter Temperaturverläufe angestellt. Für das in Abbildung 4.6 dargestellte Beispiel ist der Hintergrundvolumenfluß von $40 \mathrm{ml} / \mathrm{min}$ für eine Sekunde verzehnfacht worden. Die abgebildeten Temperaturprofile in einer Höhe von $600 \mathrm{~mm}$ beziehen sich auf die jeweils breiteste Stelle der Welle. Da es meßtechnisch schwierig ist, diese Stelle beim Wellendurchlauf genau zu treffen, werden in aller Regel mehrere Profile hintereinander aufgenommen und im Anschluß das Profil mit der größten Zentrumstemperatur für die Vergleiche herangezogen. Die Übereinstimmung zwischen den numerischen und den experimentellen Profilen ist ähnlich gut wie bei den stationären Kanälen. Die Temperaturmeßmethode erweist sich also auch für die Vermessung der instationären Wellenphänomene als geeignet.

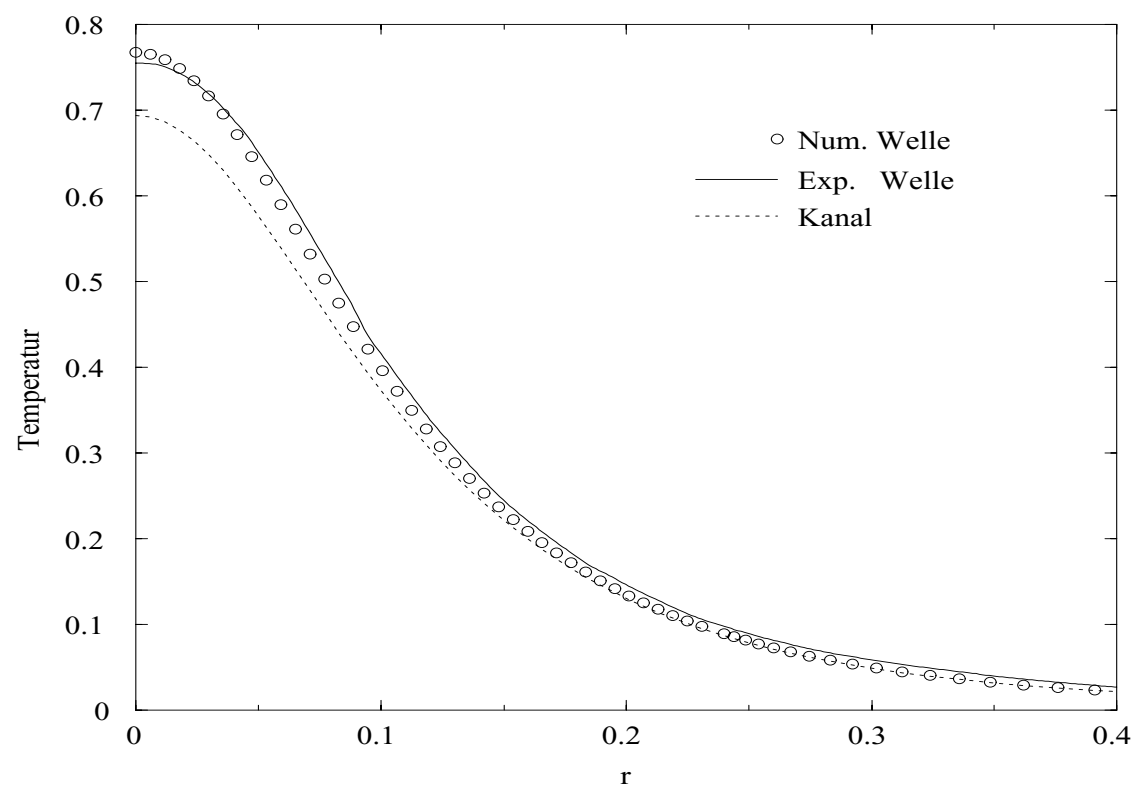

Abbildung 4.6: Vergleich zwischen einem numerisch (Kreise) und einem experimentell (durchgezogen) bestimmtem Temperaturprofil an der breitesten Stelle einer aufsteigenden Welle in 600 mm Höhe. Das gestrichelte Profil gibt die Temperaturverteilung im stationären Kanal in gleicher Aufstiegshöhe an. 
Abbildung 4.7 zeigt zwei weitere experimentell bestimmte radiale Temperaturverläufe in einer aufsteigenden Welle in zwei unterschiedlichen Höhen im Vergleich zur Kanaltemperatur. Da das Wellenmaterial am Einströmpunkt die gleiche Temperatur hat wie das des Hintergrundvolumenflusses, besteht das thermische Erscheinungsbild der Wellen in niedrigen Aufstiegshöhen (z.B. Abbildung 4.7 / Graph $150 \mathrm{~mm}$ ) hauptsächlich aus einer Verbreiterung des stationären Temperaturverlaufs. Erst bei größeren Aufstiegshöhen macht sich die stärkere Auskühlung des stationären Kanals im Vergleich zur Welle bemerkbar, so daß hier eine deutliche Temperaturerhöhung im Zentrum der Welle im Vergleich zum Kanal auftritt (z.B. Abbildung 4.7 / Graph $750 \mathrm{~mm}$ ). Des weiteren beobachtet man im Wellenzentrum einen annähernd konstanten radialen Temperaturverlauf, der auf die welleninterne Zirkulation (vergl. Abbildung 2.3) und die damit verbundene advektive Durchmischung des heißen Materials zurückzuführen ist.

In Abbildung 4.8 a) ist eine experimentell bestimmte, vollständige thermische Struktur einer aufsteigenden Welle dargestellt. Bei derartigen Messungen werden die Laserstrahlablenkungswinkel in einer festen Meßhöhe während des Wellendurchlaufs kontinuierlich aufgezeichnet. Für die Darstellung sind die aus den Ablenkungswinkeln bestimmten Einzelprofile unter Berücksichtigung der Wellengeschwindigkeit (zur Skalierung der z-Koordinate) zusammengesetzt worden. Da die Meßhöhe nicht variiert worden ist, zeigt Abbildung 4.8 a) strenggenommen nicht das Temperaturfeld der Welle, sondern vielmehr den zeitlichen Verlauf der radialen Temperaturprofile in einer konstanten Höhe. Da sich die Temperatur in der Welle während der Meßdauer (bzw. des Wellendurchlaufs durch den Meßstrahl) nur unwe-

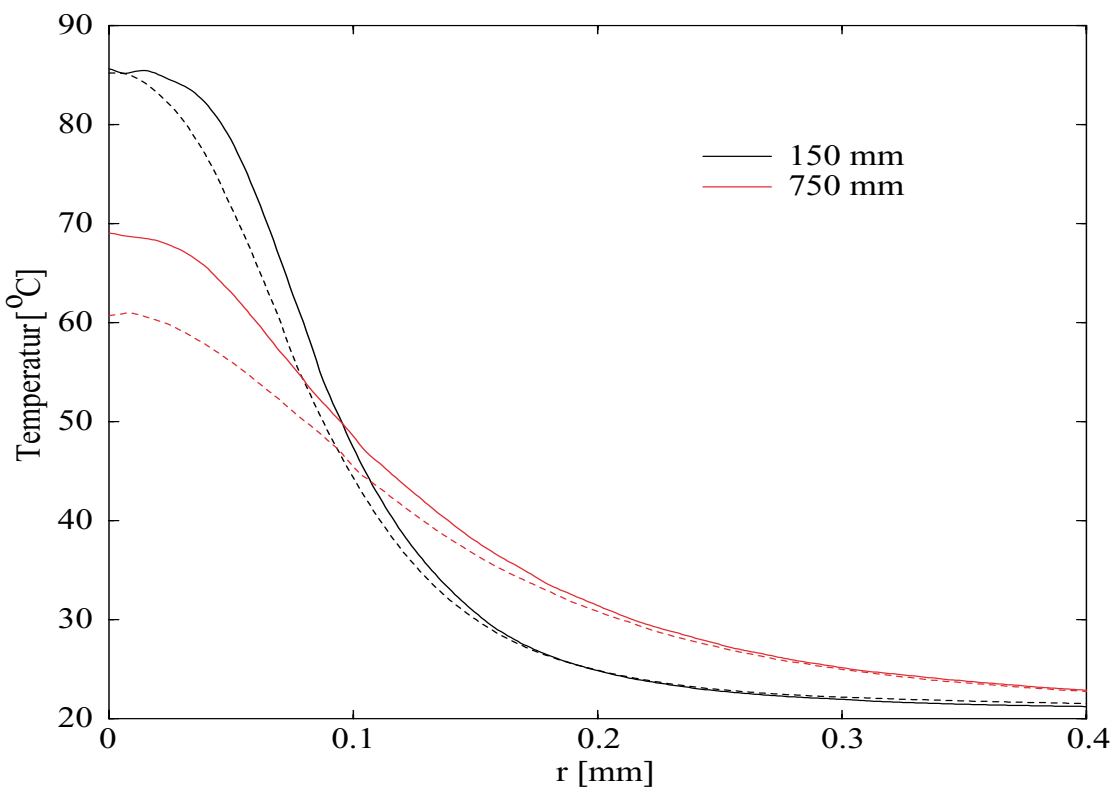

Abbildung 4.7: Vergleich von experimentell bestimmten radialen Temperaturprofilen von einer aufsteigenden Welle (durchgezogen) mit den Temperaturen im stationären Kanal (gestrichelt) für zwei verschiedene Aufstiegshöhen. 


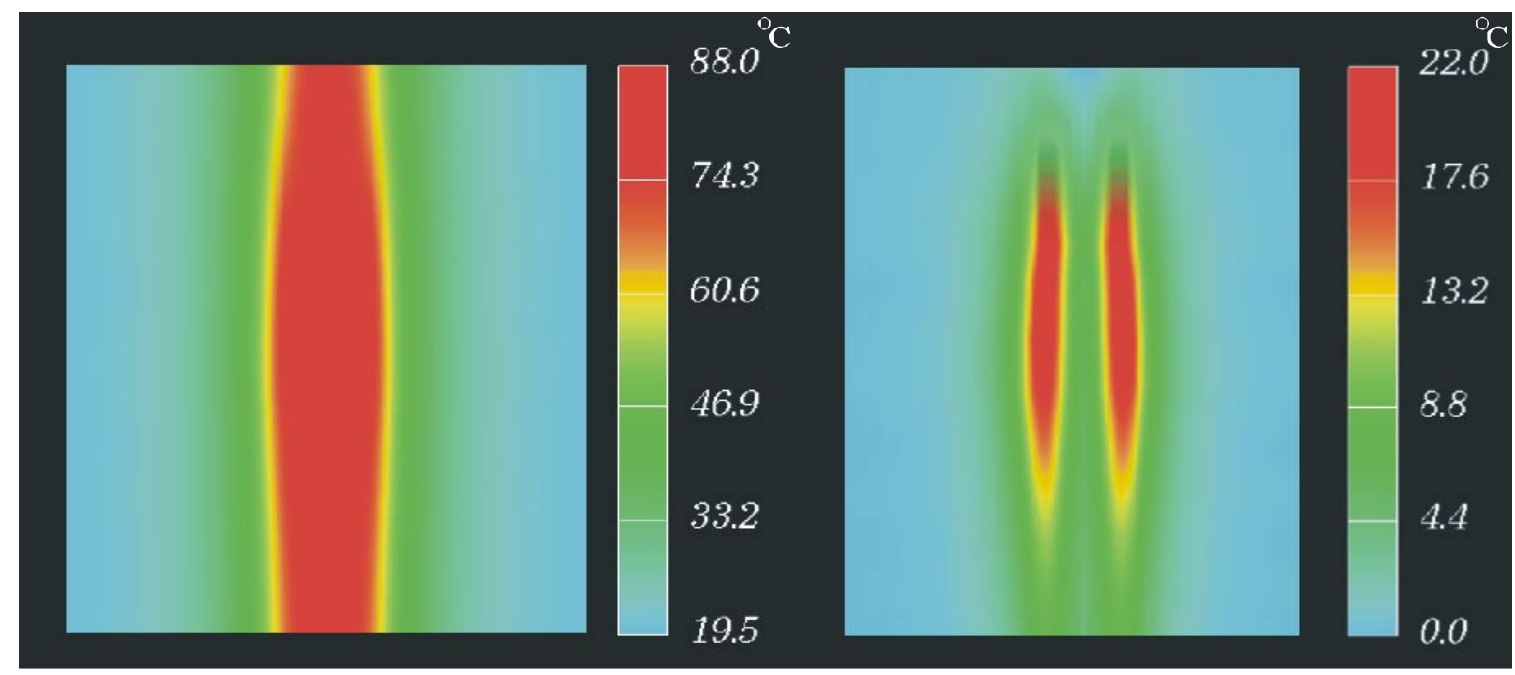

a)

b)

Abbildung 4.8: Vollständig ausgemessene Temperaturstruktur einer aufsteigenden Welle (a) im Höhenbereich zwischen 535-665 mm Aufstiegshöhe und Differenztemperaturfeld im Vergleich zum stationären Kanal (b) in gleicher Höhe.

sentlich ändert, können die Ergebnisse als gute Approximation für die thermische Struktur der Welle aufgefaßt werden. In Abbildung 4.8 b) ist das Differenztemperaturfeld zwischen den Temperaturen der Welle und der Kanaltemperatur in $600 \mathrm{~mm}$ Höhe dargestellt. Da der Plume in diesem Experiment mit einem Hintergrundvolumenfluß von $60 \mathrm{ml} / \mathrm{min}$ und einer Temperaturanomalie des injizierten Materials am Einströmpunkt von $70 \mathrm{~K}$ relativ stark ist, kühlt der Kanal auch in größeren Aufstiegshöhen nur geringfügig aus. Deshalb ist die thermische Struktur der Welle neben einer leichten Erhöhung der Maximaltemperatur hauptsächlich durch eine Verbreiterung der radialen Temperaturverläufe im Vergleich zum Kanal gekennzeichnet.

Abbildung 4.9 zeigt die Sequenz aus Differenztemperaturfeldern (stationäres Temperaturfeld ist subtrahiert worden) eines numerisch modellierten Wellenaufstiegs zu verschiedenen Zeitpunkten. Die Darstellung als Differenztemperaturfeld hat den Vorteil, daß die Welle besser zu lokalisieren und leichter von Kanal und Matrix abzugrenzen ist.

Für die hier abgebildeten Resultate ist in einem stationären Plume mit einem Volumenfluß von $60 \mathrm{ml} / \mathrm{min}$ und einer Temperaturanomalie von $75 \mathrm{~K}$ der Volumenstrom für zwei Sekunden um den Faktor 20 erhöht worden. Es zeigt sich, daß das in Abbildung 4.8 b) experimentell bestimmte thermische Erscheinungsbild der Welle in Form einer Kanalverbreiterung auch in den numerischen Studien zu beobachten ist. In der numerischen Sequenz 4.9 nimmt die Verbreiterung mit zunehmender Aufstiegshöhe ab (Abbildung $4.9 \mathrm{a}-\mathrm{c}$ ) und das Maximum der Temperaturanomalie konzentriert sich zunehmend im Wellenzentrum (Abbildung $4.9 \mathrm{~d}$ und e). 

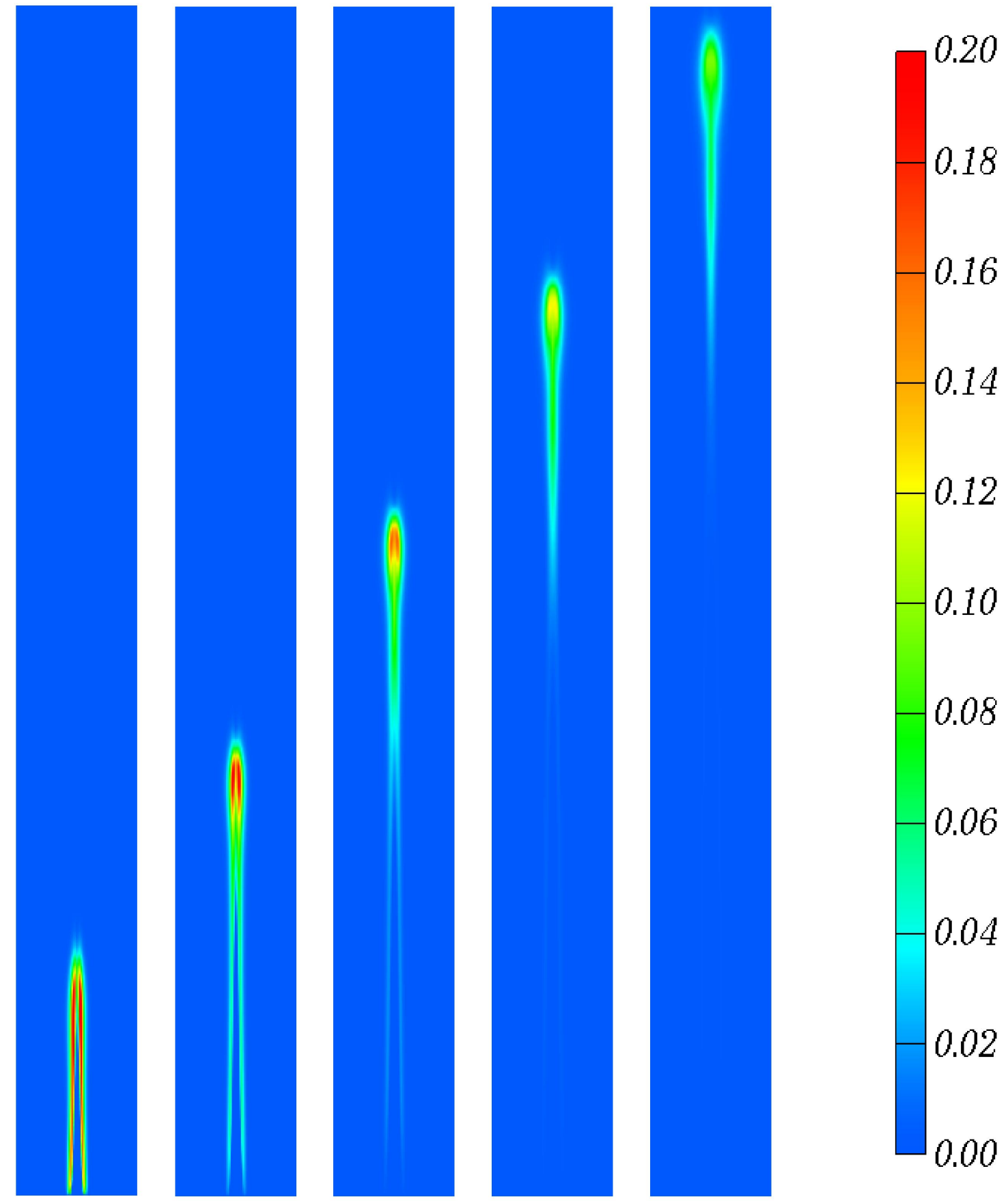

a)

b)

c)

d)

e)

Abbildung 4.9: Dimensionslose Differenztemperaturfelder im Vergleich zum stationären Kanal einer aufsteigenden Welle (numerisch) zu verschiedenen Zeitpunkten. Auf das Laborexperiment bezogen beträgt in dieser Rechnung der Hintergrundvolumenfluß $60 \mathrm{ml} / \mathrm{min}$ mit einer Temperaturanomalie am Einströmpunkt von umgerechnet $75 \mathrm{~K}$. Die Volumenstörung wurde durch eine zwei Sekunden (bezogen auf experimentelle Zeitskala) andauernde Erhöhung der Volumenflusses um den Faktor 20 erzeugt. Verstrichene Zeit seit Wellenerzeugung bezogen auf experimentelle Zeitskala: a) $16 \mathrm{~s}$; b) $43 \mathrm{~s}$; c) $83 \mathrm{~s}$; d) $143 \mathrm{~s}$; e) 227 s. Dargestellte Zylindergröße $0.2 \times 2 \mathrm{~m}$. 
Die langgezogene Temperaturanomalie hinter der Welle (vergl. insbesondere Abbildung $4.9 \mathrm{a}-\mathrm{c}$ ) wird ebenfalls auf eine Kanalverbreiterung zurückgeführt, die sich während des Wellendurchlaufs ausbildet. In der Differenztemperaturdarstellung macht sich diese Verbreiterung als eine Art „Doppelschweif“ bemerkbar, da die Welle heißes Kanalmaterial nach außen drängt, so daß eine schlauchartige Temperaturanomalie auftritt. Diese Anomalie klingt nur langsam ab, weil in den Bereichen hinter der Welle keine radialen Geschwindigkeiten auftreten (vergl. Darstellung 4.12 b im folgenden Abschnitt), so daß der radiale Wärmetransport rein diffusiv abläuft. Ansatzweise erkennt man diese Art von Temperaturanomalie auch in wesentlich weniger stark ausgeprägter Form unmittelbar oberhalb der Welle, wo die Verbreiterung ihren Ausgang nimmt.

\section{Bevorzugte Wellenform}

Eine weitere aus geophysikalischer Sicht sehr wichtige Welleneigenschaft ist in Abbildung 4.10 dargestellt. Die hier abgebildeten Differenztemperaturfelder zweier Wellen sind nahezu identisch, obwohl die Dauer der wellengenerierenden Störung jeweils unterschied-

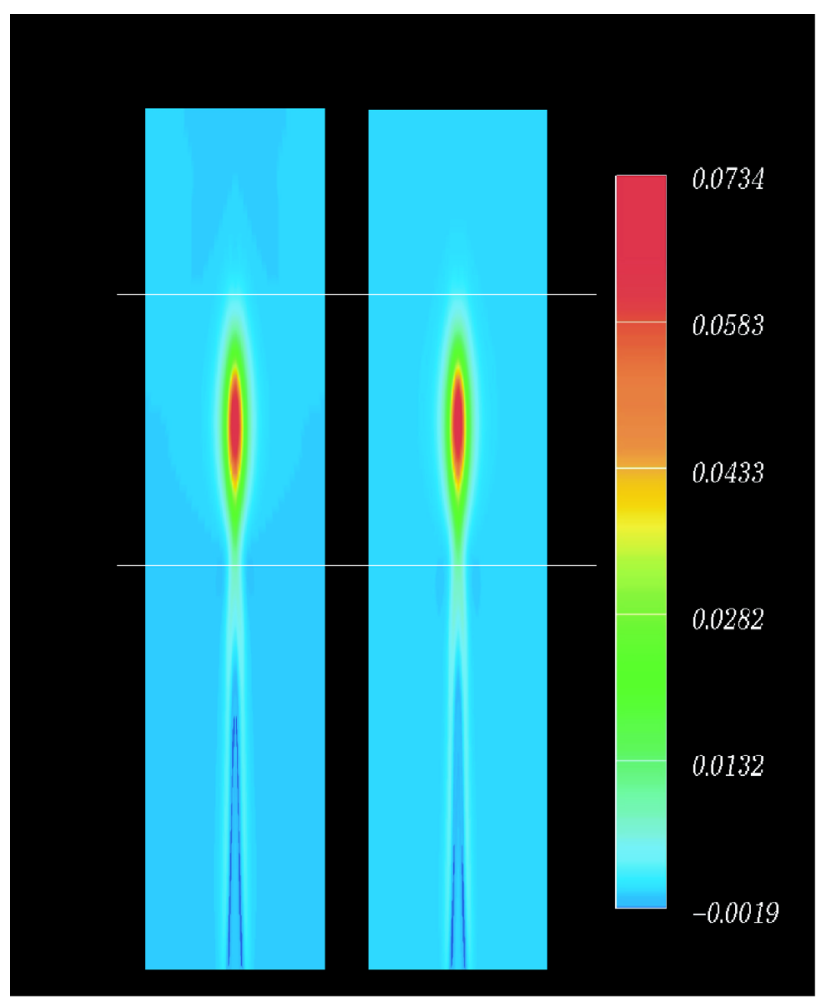

Abbildung 4.10: Differenztemperaturfelder zweier Wellen, erzeugt durch Volumenstörungen unterschiedlicher Dauer. Für die linke Welle ist der Volumenstrom um den Faktor 7.5 für $1.33 \mathrm{~s}$ und für die rechte Welle um den Faktor 25 für $0.4 \mathrm{~s}$ erhöht worden. Die Menge des zusätzlichen injizierten Materials ist in beiden Fällen gleich. Beide Wellen haben identische Aufstiegsgeschwindigkeiten. 
lich gewählt worden ist. Lediglich die Menge des zusätzlich injizierten Materials ist in beiden Fällen gleich. Für die Erzeugung der linken Welle ist der Volumenfluß um den Faktor 7.5 für $1.33 \mathrm{~s}$ (skaliert auf ein vergleichbares Laborexperiment) erhöht worden. Bei der rechten Welle ist der Faktor der Volumenflußerhöhung auf 25 und die Dauer der Erhöhung auf $0.4 \mathrm{~s}$ gesetzt worden. Neben dem thermischen Erscheinungsbild der Wellen ist auch die Aufstiegsgeschwindigkeit in beiden Fällen gleich groß. Diese Beobachtung zeigt, daß die Wellen unabhängig von dem wellengenerierenden Mechanismus offenbar eine bevorzugte Form und Ausbreitungsgeschwindigeit haben, die innerhalb der untersuchten Grenzen (Volumenflußerhöhungen um den Faktor 2-30) lediglich von der Menge des zusätzlich injizierten Volumens abhängen. Auch experimentell bestimmte Temperaturprofile in Wellen mit gleicher zusätzlich injizierter Materialmenge aber unterschiedlichem zeitlichen Verlauf der Störung zeigen ein identisches Aussehen.

Die hier beschriebene Beobachtung bestätigt zum einen den solitären Charakter der in den Modellierungen auftretenden Wellen und stützt zum anderen die Vermutung, daß es auch in den thermischen Plumes der Erde solitäre Wellen geben könnte. Dort müßten die Wellen durch Volumenfluktuationen aus einer thermischen Grenzschicht heraus entstehen, was wahrscheinlich mit sehr unterschiedlichen Volumenflußerhöhungen bzw. zeitlich sehr variablen Störungen verbunden wäre. Die Tatsache, daß es relativ unerheblich erscheint, mit welchem zeitlichen Verlauf das zusätzliche Material vom Plumekanal aufgenommen wird, macht eine Entstehung der Wellen in den thermischen Plumes der Erde deutlich wahrscheinlicher.

\subsubsection{Geschwindigkeiten}

Betrachtet man die Geschwindigkeiten im Zusammenhang mit den aufsteigenden Wellen, so muß man zwischen der Materialgeschwindigkeit in den Wellen und der Geschwindigkeit der Gesamtwelle unterscheiden. Aus geophysikalischer Sicht, insbesondere im Hinblick auf einen zeitlich variablen Hotspot-Vulkanismus ist die Wellengeschwindigkeit gegenüber der Teilchengeschwindigkeit die relevantere Größe, da sie die Zeitskala bestimmt, die eine Welle benötigt um die Lithosphäre zu erreichen.

Experimentell kann die Wellengeschwindigkeit z.B. über eine Schattenrißmethode bestimmt werden. Dazu mißt man die Zeit, die der auf die Beobachtungsfläche projizierte Wellenschatten benötigt, um eine definierte Strecke zurückzulegen. Dabei tritt allerdings das Problem auf, daß die Welle im Schattenbild kaum auszumachen ist, wenn sich ihre Temperatur von der des Kanals nur unwesentlich unterscheidet. Daher konnte diese Methode weder in niedrigen Aufstiegshöhen noch bei großem Hintergrundvolumenfluß erfolgreich angewendet werden. In diesen Fällen ist, wie zuvor diskutiert (Kapitel 4.2.1), das thermische Erscheinungsbild der Welle hauptsächlich durch eine Kanalverbreiterung gekennzeichnet, die nur schwer zu beobachtende langgezogene vertikale Strukturen im Schattenbild erzeugt.

In den numerischen Rechnungen wird zur Bestimmung der Wellengeschwindigkeiten die Aufstiegsgeschwindigkeit des Temperaturanomalieschwerpunkts der Welle ermittelt. Abbil- 
dung 4.11 zeigt beispielhaft die numerisch bestimmten Aufstiegsgeschwindigkeiten von unterschiedlich großen Wellen in zwei verschiedenen stationären Kanälen mit einem Hintergrundvolumenfluß von $40 \mathrm{ml} / \mathrm{min}$ (Abbildung 4.11 a) bzw. $80 \mathrm{ml} / \mathrm{min}$ (Abbildung $4.11 \mathrm{~b}$ ). In beiden Rechnungen beträgt die Temperaturanomalie des injizierten Materials $75 \mathrm{~K}$.

Man erkennt, daß Wellen, die durch größere Volumenstörungen erzeugt werden, schnellere Aufstiegsgeschwindigkeiten besitzen. Außerdem zeigt sich, daß die Wellen in dem Plume mit niedrigem Hintergrundvolumenfluß aufgrund der Auskühlung des Kanals eine größere relative Geschwindigkeitsabnahme zeigen (Abbildung 4.11 a), als die Wellen in dem „starken“ Plume, der den Wellen über größere Aufstiegshöhen hinweg konstantere Bedingungen bietet (Abbildung 4.11 b). Die absolute Wellengeschwindigkeit wird in erster Linie von der thermischen Struktur des Plumekanals und der Menge des zusätzlich injizierten Materials bestimmt. In Kapitel 5.1 werden diese Abhängigkeiten durch systematische Parameterstudien und die Bildung von Skalierungsgesetzen genauer untersucht.

Zusätzlich zu den Beispielen für die Wellengeschwindigkeiten sollen hier auch Ergebnisse von experimentell bestimmten Teilchengeschwindigkeiten dargestellt werden, weil sie ein weiteres Mal den direkten Vergleich zwischen Numerik und Experiment ermöglichen. Zudem machen sie die lokalen Strömungsvorgänge im Zusammenhang mit dem Wellenaufstieg besser verständlich.

In Abbildung 4.12 sind zunächst numerisch bestimmte Strömungsgeschwindigkeiten in vertikaler und radialer Richtung dargestellt, die die prinzipielle Geschwindigkeitsstruktur in einer Welle verdeutlichen. Die Vertikalgeschwindigkeit (Abbildung 4.12 a) zeichnet sich in-
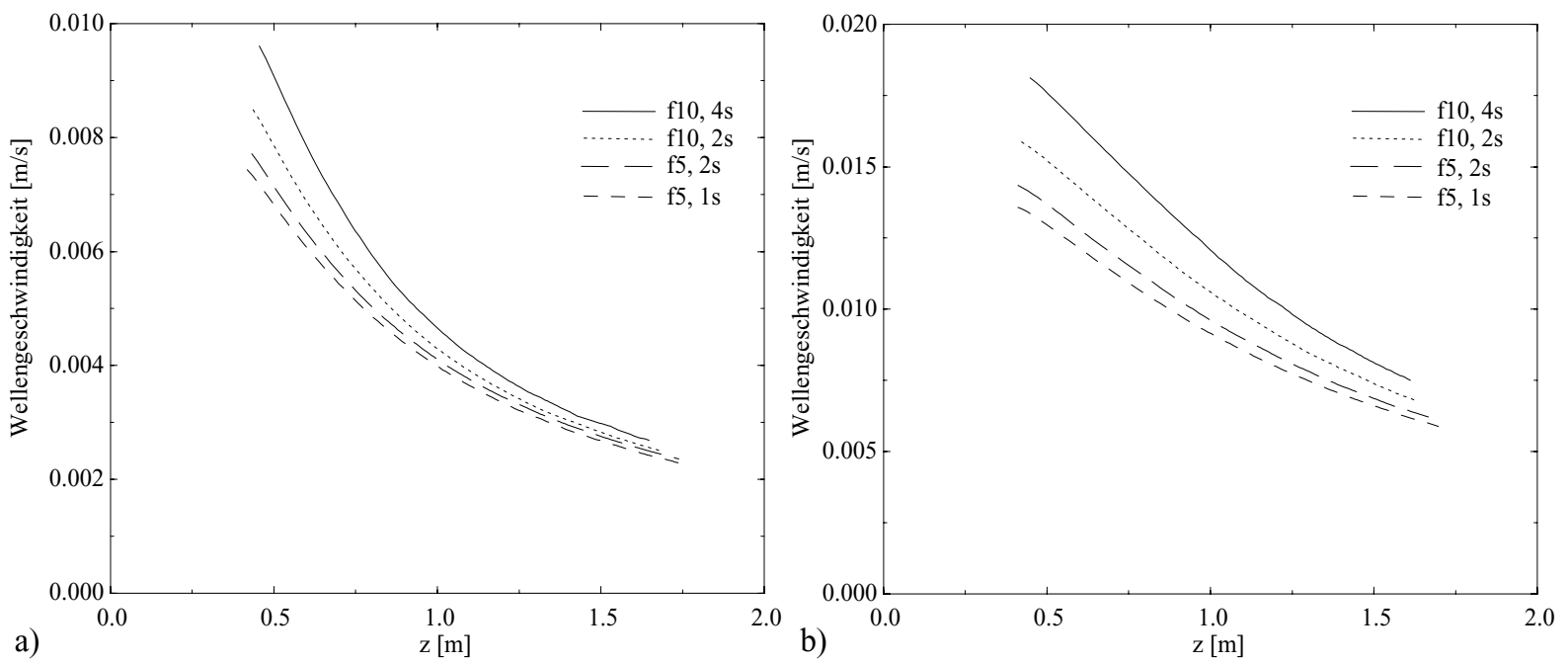

Abbildung 4.11: Numerisch bestimmte Wellengeschwindigkeiten in einem stationären Kanal mit $40 \mathrm{ml} / \mathrm{min}$ (a) und $80 \mathrm{ml} / \mathrm{min}$ (b) Hintergrundvolumenfluß und einer Temperaturanomalie von $75 \mathrm{~K}$. In der Legende bezeichnet die Zahl hinter dem $\mathrm{f}$ den Faktor der Volumenflußerhöhung. Dahinter ist die Dauer der erhöhten Flusses angegeben. 
nerhalb der Welle durch sehr große radiale Gradienten aus. Das hängt damit zusammen, daß die Welle in einem mitbewegten Koordinatensystem die in Abbildung $2.3 \mathrm{zu}$ beobachtende interne Zirkulation aufweist, die die vertikalen Geschwindigkeiten in einem festen Koordinatensystem nach außen hin stark abfallen läßt. Im ortsfesten Koordinatensystem tritt kein Rückstrom von Wellenmaterial auf.

Die Darstellung der radialen Geschwindigkeiten (Abbildung 4.12 b) stützt die im Zusammenhang mit Abbildung 4.9 angestellten Überlegungen zur thermischen Verbreiterung des Kanals. Die Bereiche mit der größten nach außen gerichteten Radialgeschwindigkeit eilen dem Maximum der Vertikalgeschwindigkeit und damit dem Wellenzentrum voraus. Dadurch wird im oberen Bereich der Welle Material nach außen transportiert, was zu den diskutierten Temperaturanomalien führt. Im Bereich der verschwindenden radialen Geschwindigkeit hat die Welle ihre größte radiale Ausdehnung. Der nach innen gerichtete Materialrückstrom im unteren Bereich der Welle ist im Gegensatz zu der nach außen gerichteten Strömung großräumiger und zeigt weniger große Geschwindigkeitsamplituden.

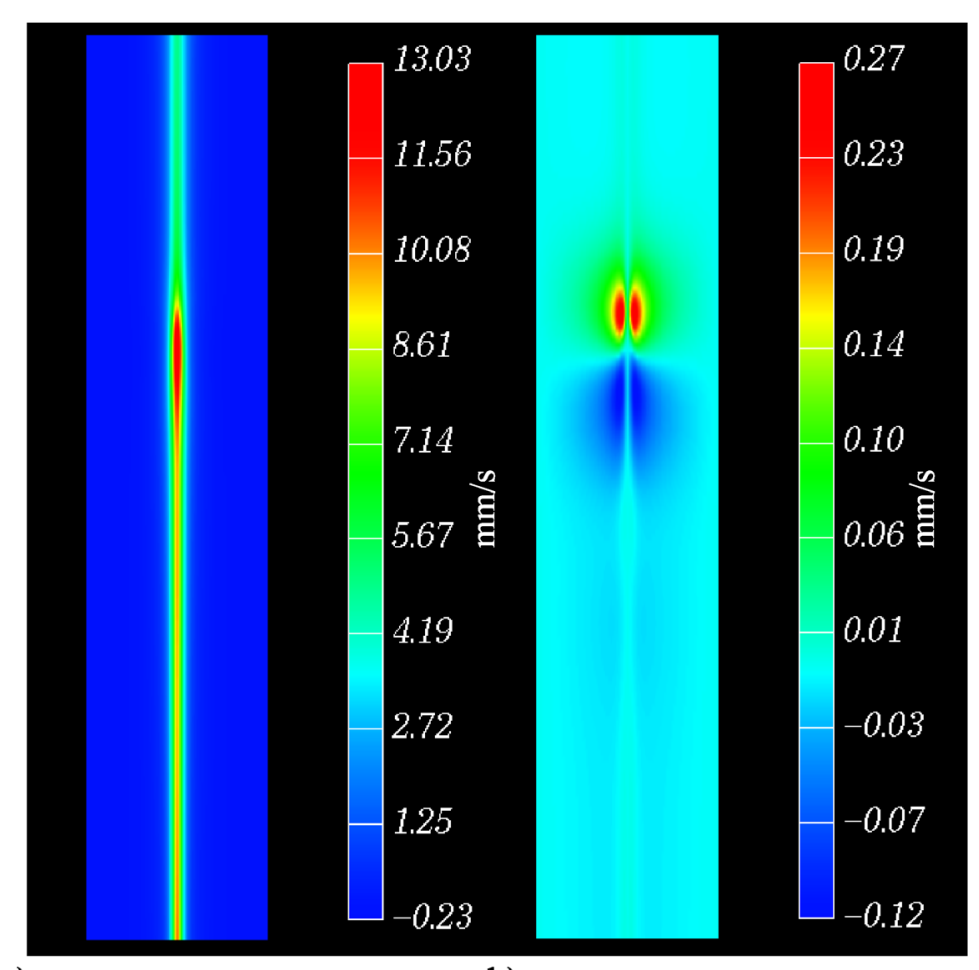

a)

b)

Abbildung 4.12: Vertikale (a) und radiale (b) Geschwindigkeitsstruktur einer aufsteigenden Welle (numerisches Resultat). Positive radiale Geschwindigkeiten sind nach außen gerichtet, negative nach innen. Die Beträge der Geschwindigkeiten sind gemäß eines vergleichbaren Laborexperiments skaliert worden. Zur Erzeugung der Welle ist der Hintergrundvolumenfluß von $80 \mathrm{ml} / \mathrm{min}$ (Temperaturanomalie des einströmenden Materials $75 \mathrm{~K}$ ) um den Faktor 20 für 2 Sekunden erhöht worden. Dargestellter Zylinderausschnitt $0.2 \times 1 \mathrm{~m}$. 
In Abbildung 4.13 werden die experimentell (a) und numerisch (b) bestimmten Vertikalgeschwindigkeiten in einer aufsteigenden Welle verglichen. Da aus technischen Gründen der Bildausschnitt der PIV-Aufnahmen für eine komplette Wellendarstellung nicht groß genug gewählt werden konnte, findet man hier nur das Geschwindigkeitsfeld des oberen Wellenbereiches ${ }^{1}$. Man erkennt, daß die durch die PIV-Methode bestimmten vertikalen Strömungsgeschwindigkeiten deutlich größer sind als die numerisch ermittelten. Angesichts der guten Übereinstimmung für die Messungen im stationären Kanal ist diese Abweichung zunächst etwas überraschend. Sie erklärt sich jedoch aus der Tatsache, daß während der PIVExperimente sehr viele Wellen (bis zu 15) erzeugt worden sind und offenbar der Einfluß der vorher durch den Kanal hindurchlaufenden Wellen unterschätzt wurde. Vermutlich ist der zeitliche Abstand zur vorhergehenden Welle nicht groß genug gewählt worden, so daß die im Zusammenhang mit Abbildung 4.9 diskutierte „,doppelschweifartige“ Temperaturanomalie nicht weit genug abklingen konnte. Dadurch hat sich die ausgemessene Welle in einem ,thermisch breiteren“ Kanal deutlich schneller ausbreiten können, was schnellere Teilchengeschwindigkeiten in der Welle zur Folge hatte. Diese Überlegung wird auch durch die in Abbildung 4.14 dargestellte numerisch bestimmte Aufstiegssequenz bestätigt. Um den Einfluß einer durch den Kanal laufenden Welle auf eine nachfolgende zu untersuchen, sind

${ }^{1}$ Bei Aufnahme einer vollständigen Welle hat die Auflösung der verwendeten CCD-Kamera nicht mehr ausgereicht, um die sehr starken radialen Geschwindigkeitsgradienten in der Welle aufzulösen.

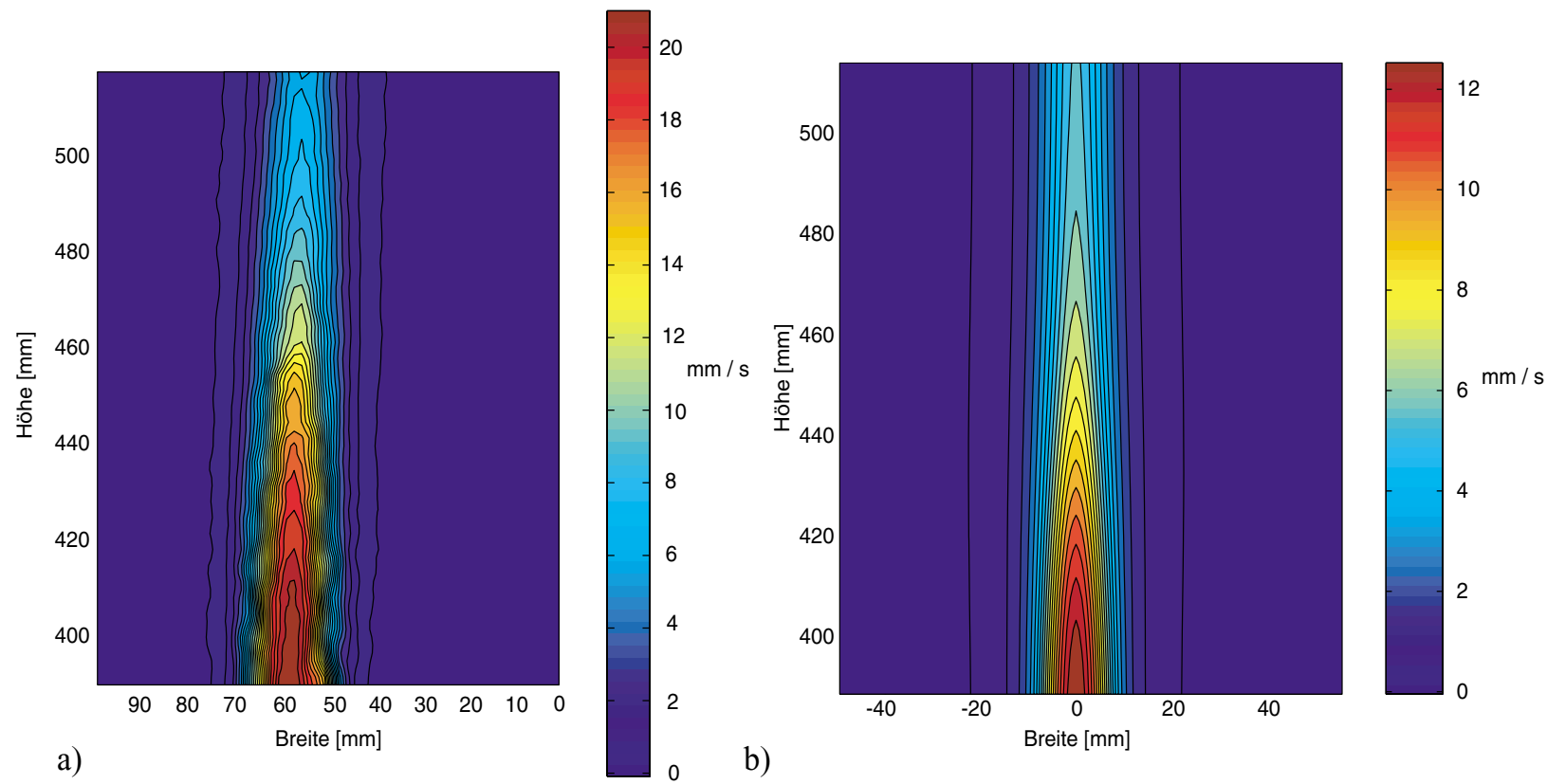

Abbildung 4.13: Vergleich zwischen einem gemessenen (a) und einem numerisch bestimmten (b) vertikalen Geschwindigkeitsfeld im oberen Bereich einer aufsteigenden Welle. Der stationäre Plumekanal hat einen Volumenfluß von $60 \mathrm{ml} / \mathrm{min}$, die Temperaturanomalie beträgt $66 \mathrm{~K}$. Der Volumenfluß ist um den Faktor 20 für 2 Sekunden erhöht worden. 


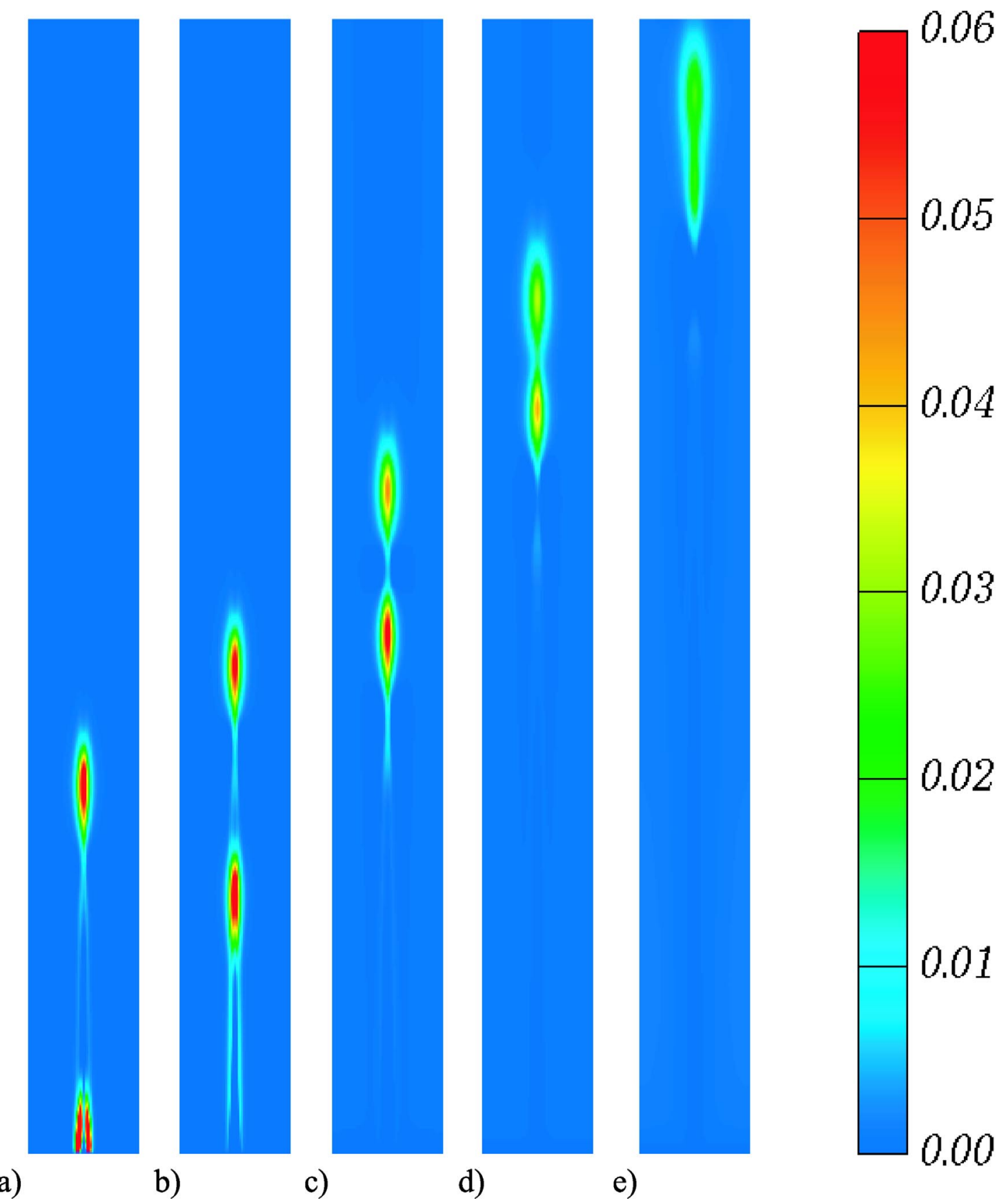

Abbildung 4.14: Dimensionslose Differenztemperaturfelder (stationäres Temperaturfeld subtrahiert) von zwei hintereinander aufsteigenden gleichgroßen Wellen zu verschiedenen Zeitpunkten. Auf das Laborexperiment bezogen beträgt in dieser Rechnung der Hintergrundvolumenfluß 40 ml/min mit einer Temperaturanomalie am Einströmpunkt von umgerechnet $75 \mathrm{~K}$. Die Volumenstörungen wurden jeweils durch eine Sekunde (bezogen auf experimentelle Zeitskala) andauernde Erhöhungen des Volumenflusses um den Faktor 10 erzeugt. Verstrichene Zeit seit Wellenerzeugung der zweiten Welle bezogen auf experimentelle Zeitskala: a) $13 \mathrm{~s}$; b) $64 \mathrm{~s}$; c) $168 \mathrm{~s}$; d) $316 \mathrm{~s}$; e) $519 \mathrm{~s}$. Dargestellte Zylindergröße $0.2 \times 2 \mathrm{~m}$. 
zwei Wellen auf gleiche Art in einem kurzen Abstand hintereinander erzeugt worden. Die Abbildung zeigt erneut Differenztemperaturfelder zum stationären Kanal. Die zweite Welle profitiert in diesem Fall deutlich von der durch die erste Welle erzeugten thermischen Verbreiterung, so daß es ihr gelingt die voranlaufende Welle einzuholen. In der experimentellen Messung beobachtet man nicht nur größere Vertikalgeschwindigkeiten, sondern auch größere Horizontal- bzw. Radialkomponenten (Abbildung 4.15²).

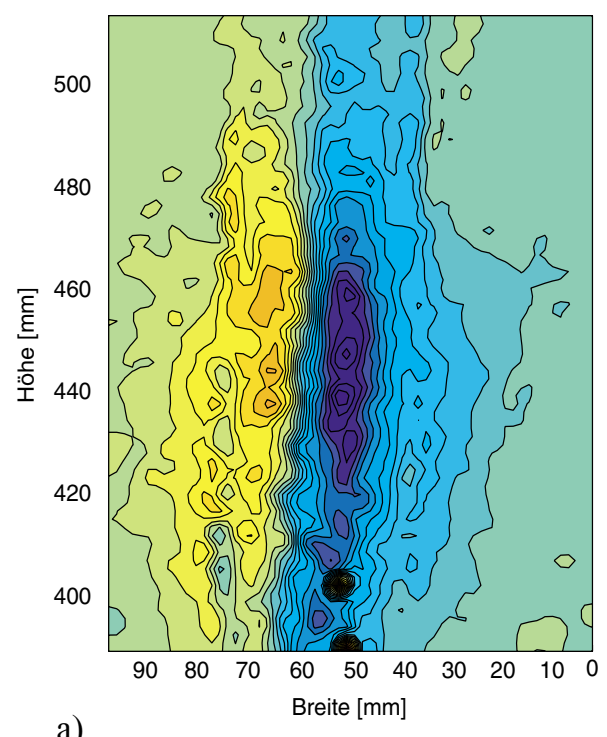

a)

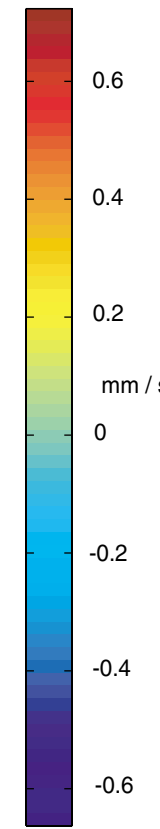

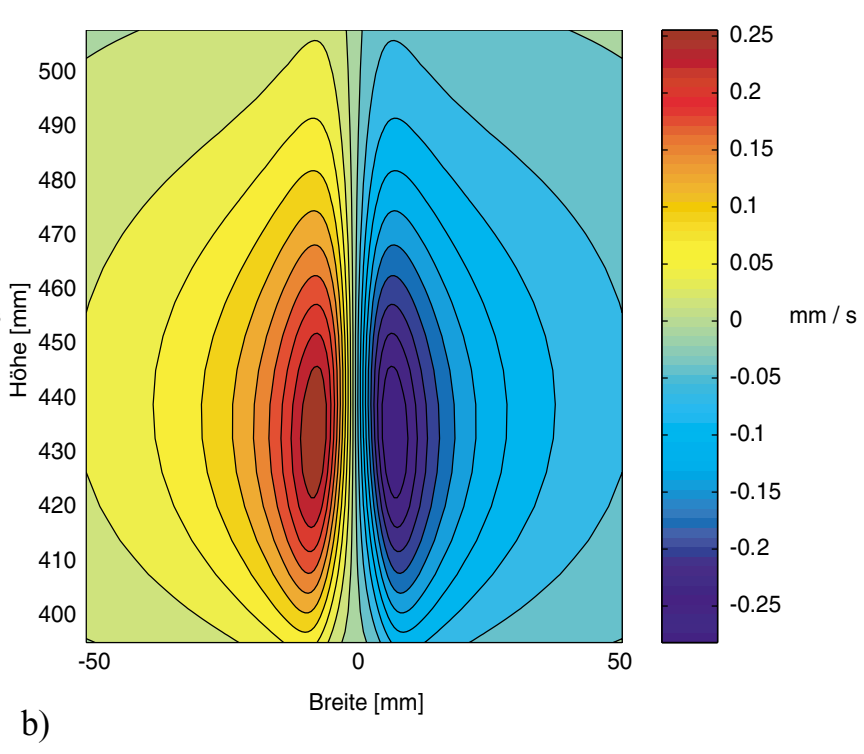

Abbildung 4.15: Der zur Abbildung 4.13 analoge Vergleich der Radial- bzw. Horizontalkomponente. a) PIV-Messung ungefiltert und nicht geglättet, b) numerisches Resultat.

Die experimentell bestimmten radialen Geschwindigkeitswerte sind im Vergleich zur Vertikalkomponente (Abbildung 4.13 a) stark verrauscht, was auf die um zwei Größenordnungen kleineren Amplituden zurückzuführen ist. Die groben Formen der Geschwindigkeitsstrukturen stimmen jedoch, wenn auch nicht so überzeugend wie im Fall der Vertikalkomponente, mit den numerisch ermittelten überein.

Zusammenfassend läßt sich feststellen, daß sich, abgesehen von den diskutierten Abweichungen, experimentelle Volumenstörungen mit den gleichen wellenartigen Eigenschaften ausbreiten wie numerische. Die Wellen zeigen in beiden Modellierungen vergleichbare Strö-

\footnotetext{
${ }^{1}$ Man beachte den Unterschied in der Darstellung im Vergleich zu den numerisch bestimmten Radialgeschwindigkeiten: Da bei den durchgeführten PIV-Aufnahmen mit dem Lichtschnitt eine senkrechte „Meßebene“ durch das Plumezentrum gelegt wird, sind die radialen Geschwindigkeiten in den PIV-Ergebnissen als horizontale Geschwindigkeiten in der Beobachtungsebene dargestellt, so daß eine Teilchenbewegung nach links durch ein positives und eine Teilchenbewegung nach rechts durch ein negatives Vorzeichen gekennzeichnet ist. Für die direkten Vergleiche sind auch die numerischen Resultate derartig dargestellt worden.
} 
mungsstrukturen und erzeugen die gleichen Temperaturanomalien.

Wechselwirkungen zwischen zwei Wellen wurden u.a. auch von Whitehead und Helfrich [1990] untersucht. Abbildung 4.16 zeigt eine von den Autoren aufgenommene Zeitsequenz von zwei aufsteigenden Wellen in einem Plume mit chemisch bedingten Dichteunterschieden. Da die Ausbreitungsgeschwindigkeiten solitärer Wellen von ihren Amplituden abhängen, ist eine Welle mit größerer Amplitude zunächst in der Lage eine kleinere einzuholen. Danach beobachtet man ein scheinbares Überholen der kleinen Welle durch die große. Während der Kollision der Wellen findet ein Materialaustausch statt, der zu Lasten der zweiten Welle die voranlaufende anwachsen läßt, so daß diese im Umfang zunimmt und dadurch fortan schneller aufsteigen kann. Die Beobachtung zeigt, daß es sich bei diesem Vorgang strenggenommen nicht um ein „Überholen“, sondern vielmehr um einen Austausch von Material und eine damit einhergehende Veränderung der Welleneigenschaften handelt.

Um zu untersuchen, ob die in dieser Arbeit beobachteten Wellen in thermischen Plumes ähnliche Wechselwirkungen zeigen, ist für die in Abbildung 4.17 dargestellte Sequenz aus Differenztemperaturfeldern eine Welle mit doppeltem Volumeninhalt gegenüber einer voranlaufenden Welle erzeugt worden.

Erwartungsgemäß steigt die Welle mit der größeren Amplitude schneller auf und holt die erste ein (Abbildung 4.17 a-c). Nach der Kollision findet jedoch ein „Verschmelzen“ der Temperaturanomalien statt, und die Wellen sind offenbar nicht mehr in der Lage sich wieder voneinander zu trennen (Abbildung 4.17 c-e). Selbst bei Rechnungen mit noch größeren

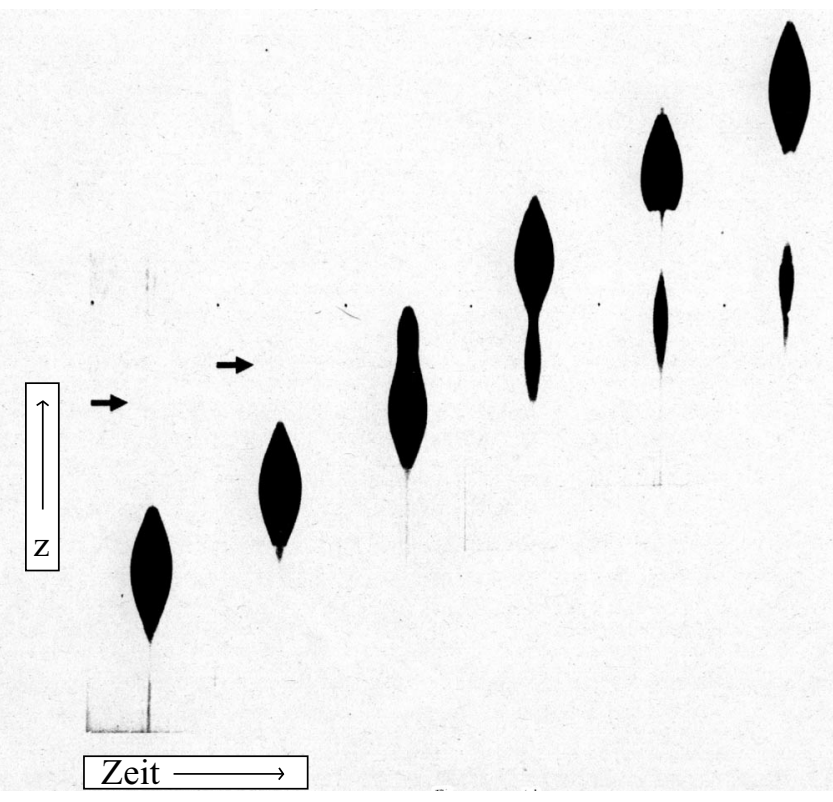

Abbildung 4.16: Wechselwirkung zwischen zwei solitären Wellen in einem chemischen Plume (aus Whitehead und Helfrich, [1990]). Die Sequenz zeigt eine kleine ungefärbte Welle (zunächst nicht sichtbar, gekennzeichnet durch Pfeile) und eine große eingefärbte Welle. Während der Kollision der Wellen findet ein Materialaustausch statt. 


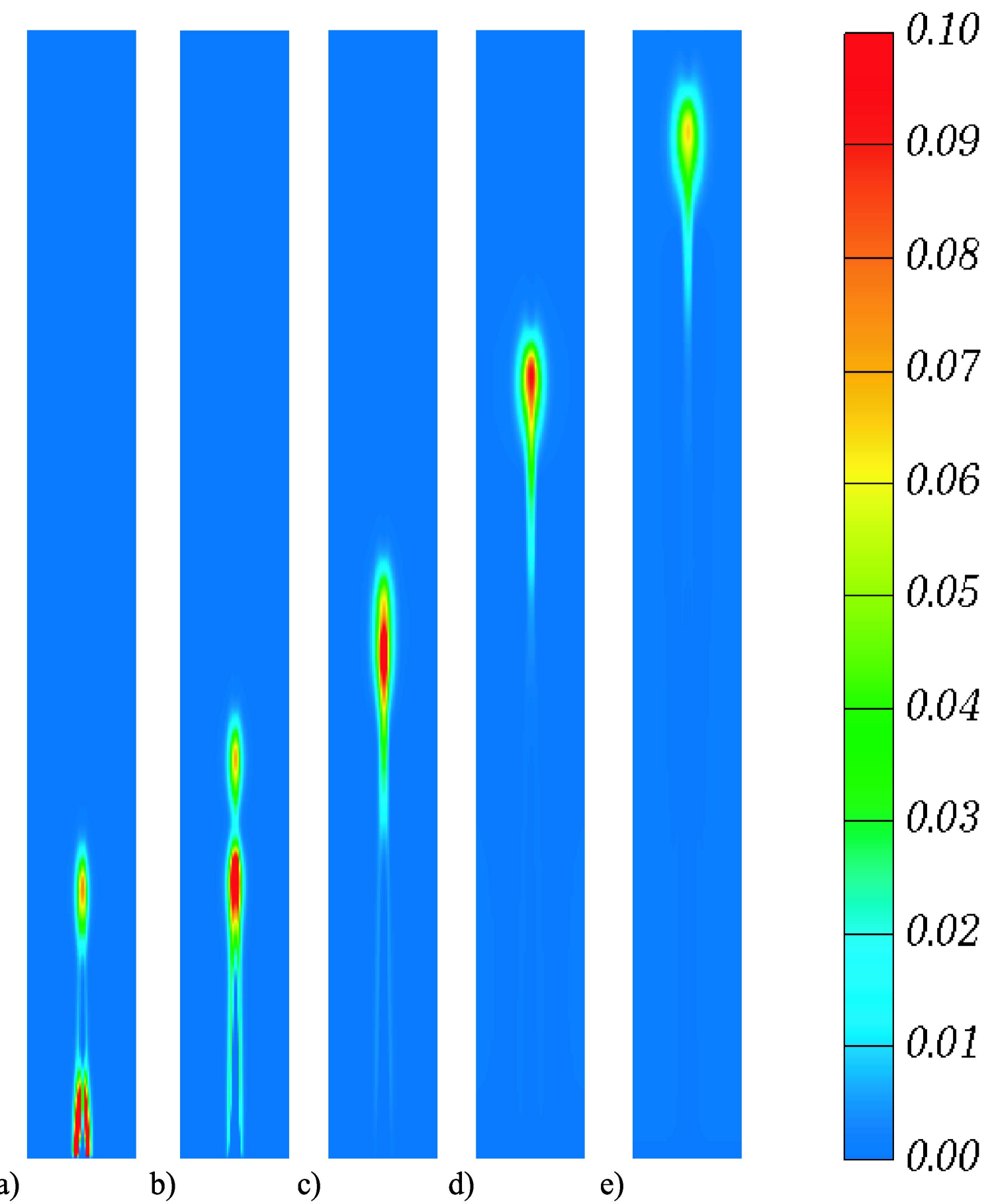

Abbildung 4.17: Dimensionslose Differenztemperaturfelder von zwei hintereinander aufsteigenden, unterschiedlich großen Wellen zu verschiedenen Zeitpunkten. Der Hintergrundvolumenfluß und die Temperaturanomalie ist identisch zu zu der in Abbildung 4.14 dargestellten Rechnung. Für die Erzeugung der ersten Welle ist der Volumenfluß für eine Sekunde um den Faktor 10, für die zweite Welle für eine Sekunde um den Faktor 25 erhöht worden. Verstrichene Zeit seit Wellenerzeugung der zweiten Welle, bezogen auf experimentelle Zeitskala: a) 29 s; b) 77 s; c) $153 \mathrm{~s}$; d) $296 \mathrm{~s}$; e) 495 s. Dargestellte Zylindergröße $0.2 \times 2 \mathrm{~m}$. 
nachfolgenden Wellen (bis zu Faktor 10 im Volumeninhalt gegenüber der ersten Welle) sind keine Ansätze für einen solchen Vorgang zu erkennen. Es wird vermutet, daß ein Auseinanderlaufen der Wellen auch bei größeren Rechengebieten bzw. Zylinderhöhen nicht zu beobachten ist. Die Ursache für dieses abweichende Verhalten der thermischen Wellen von den chemischen könnte darin liegen, daß eine voranlaufende Welle einen Kanal thermisch verbreitert, so daß der Aufstieg einer nachfolgenden Welle erleichtert wird (vergl. Diskussion im Zusammenhang mit Abbildung 4.14). Da die thermische Kanalverbreiterung unmittelbar hinter einer Welle am größten ist und diese auch nur langsam mit dem Abstand zur Welle abnimmt, würden potentiell langsamer werdende Materialpakete unterhalb der Welle sofort wieder beschleunigt und von der Welle eingefangen werden. Dieser Effekt trägt daher sehr stark zur Stabilisierung der aufsteigenden Temperaturanomalien bzw. Materialpakete bei und ist dem Auseinanderlaufen entgegengerichtet. Aus diesem Grund wird vermutet, daß es bei thermisch erzeugten Plumes grundsätzlich nicht möglich ist, daß sich einmal zusammengelaufene solitäre Wellen wieder voneinander trennen.

Im nachfolgenden Kapitel werden die hier beispielhaft angeführten Welleneigenschaften hinsichtlich Ausbreitungsgeschwindigkeit und transportierter Temperaturanomalie systematisch studiert und mit Hilfe von Skalierungsgesetzen allgemeiner dargestellt. 


\section{Kapitel 5}

\section{Ergebnisse numerischer Parameterstudien}

In diesem Kapitel werden die Ergebnisse systematischer numerischer Parameterstudien vorgestellt und in Form von Skalierungsgesetzen zusammengefaßt. Dabei wird zunächst auf die Aufstiegsgeschwindigkeit der Wellen und anschließend auf die von ihnen transportierte Temperaturanomalie eingegangen.

Danach werden Ergebnisse von Rechnungen mit tiefenabhängiger Viskosität dargestellt und deren Einfluß auf die Temperaturanomalie und die Wellenausbreitung diskutiert. Am Ende des Kapitels werden die dimensionslosen Resultate auf Plumes mit unterschiedlichen Hintergrundvolumenflüssen skaliert.

\subsection{Wellengeschwindigkeit}

Um die Abhängigkeit der Wellengeschwindigkeit von der Amplitude zu untersuchen, wurden unterschiedliche Wellen in stationären Plumekanälen erzeugt. Dabei variierten die Volumenflußerhöhungen um den Faktor 2-30. Die Dauer des erhöhten Flusses wurde zwischen 0.5-10 s variiert.

Zur Interpretation bzw. Skalierung der daraus erhaltenen Ergebnisse wird auf eine Studie von Olson und Christensen [1986] zurückgegriffen, die solitäre Wellen in chemischen Plumes sowohl experimentell als auch theoretisch untersucht. Für die analytische Betrachtung der Wellenausbreitung verwenden die Autoren ein Modell, das die in eine hochviskose Matrix eingebettete niedrigviskose Kanalströmung näherungsweise als Poiseuillesche Rohrströmung beschreibt. Dabei wird angenommen, daß sich die Breite des Kanals nur geringfügig mit der Höhe ändert, diffusive Effekte vernachlässigbar sind und die Kanalströmung laminar ist. Die daraus resultierenden Skalen sind in Anhang A.3 aufgeführt. Als Geschwindigkeitsskala wird zum Beispiel die mittlere Geschwindigkeit in einer ungestörten Kanalströmung gewählt.

Vergleiche mit experimentell erzeugten Volumenstörungen in vollentwickelten chemi- 
schen Plumekanälen zeigen, daß die Wellengeschwindigkeit systematisch von der Amplitude abhängt. Für dimensionlose Wellenvolumina kleiner 80 können die Autoren die experimentellen Ergebnisse gut durch die analytische Lösung der theoretischen Betrachtung beschreiben.

Um zu untersuchen, ob sich für thermisch erzeugte Wellen ähnliche systematische $\mathrm{Zu}$ sammenhänge zwischen Wellenamplitude und Aufstiegsgeschwindigkeit ermitteln lassen, sind die Ergebnisse der hier durchgeführten Parameterstudie mit den gleichen Größen wie bei Olson und Christensen [1986] (Anhang A.3) skaliert worden. Ein Problem dabei ist, daß die verwendeten Skalen bei thermischen Plumes höhenabhängig sind, da sich, anders als bei „,chemischen Plumes“, die in die Skalen eingehenden Viskositäts- und Dichteverhältnisse mit der Höhe ändern. Aus diesem Grund sind die Ergebnisse der Skalierung in Abbildung 5.1 nicht als Punkte, sondern als einzelne Graphen dargestellt. Mit zunehmender Aufstiegshö-

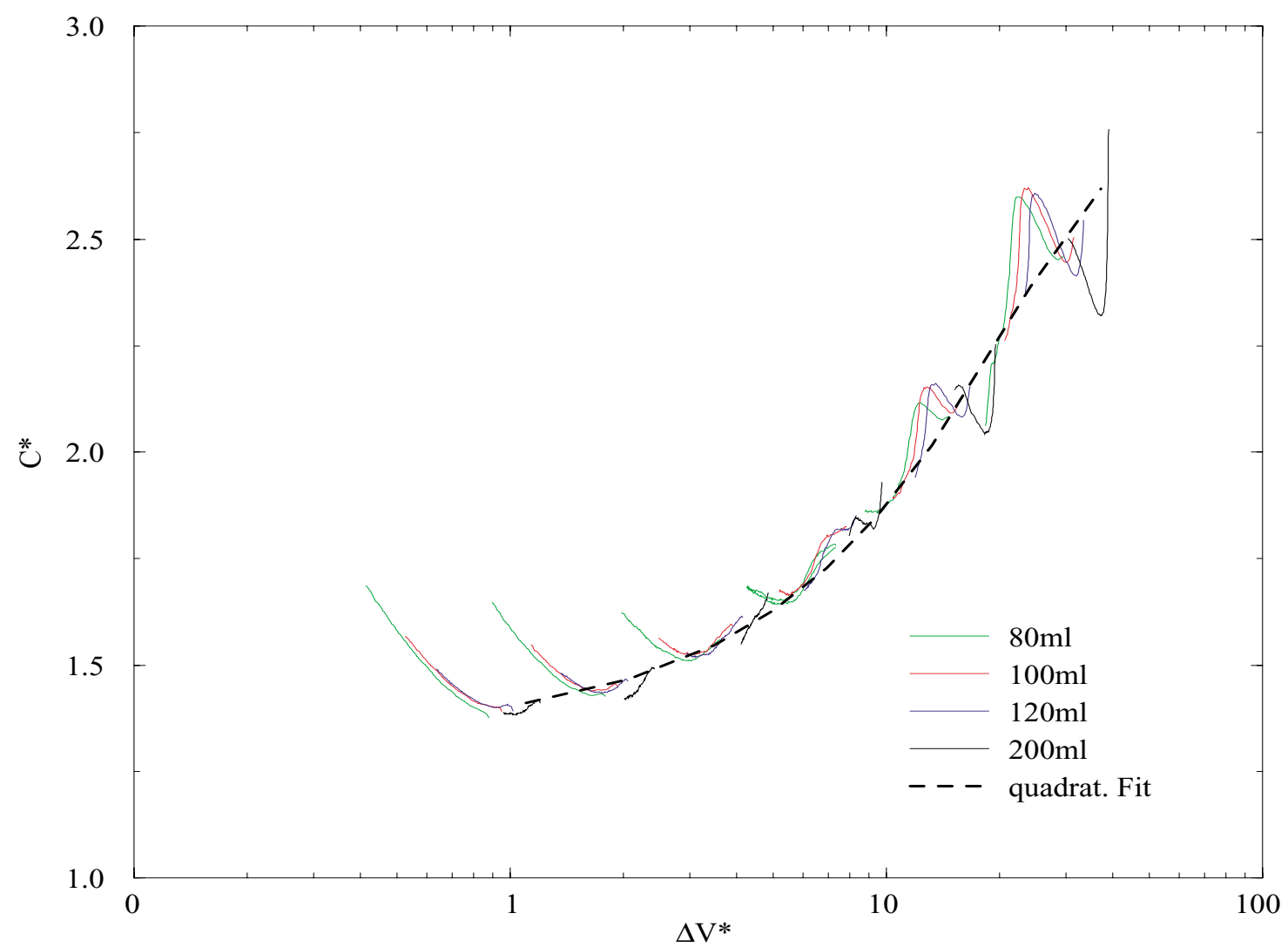

Abbildung 5.1: Dimensionslose Wellenausbreitungsgeschwindigkeit $C^{*}$ gegen dimensionsloses Wellenvolumen $\Delta V^{*}$ für thermische Plumes, bestimmt aus numerischen Parameterstudien. Das dimensionlose Wellenvolumen $\Delta V^{*}$ nimmt in größeren Aufstiegshöhen ab, so daß innerhalb eines einzelnen Graphen die Aufstiegshöhe von rechts nach links zunimmt. Ergebnisse unterschiedlicher Hintergrundvolumenflüsse sind farblich kodiert. Funktion der empirisch angebrachten Anpassung: $C_{f i t}^{*}=1.35+0.06 \Delta V^{*}-6.8 \times 10^{-4} \Delta V^{* 2}$ 
he wird das dimensionslose Wellenvolumen $\Delta V^{*}$ immer kleiner, da die Welle während des Aufstiegs thermische Energie verliert.

Abbildung 5.1 zeigt, daß sich die dimensionslosen Geschwindigkeiten $C^{*}$ der unterschiedlich großen Wellen im Bereich zwischen $\Delta V^{*}=1$ und $\Delta V^{*}=20 \mathrm{sehr}$ gut durch einen empirischen quadratischen Ausdruck anpassen lassen. Im Bereich von $\Delta V^{*}<1$ beobachtet man für kleine Hintergrundvolumenflüsse (gleiche Volumenflüsse sind mit den gleichen Farben dargestellt) ein Ansteigen der Geschwindigkeit $C^{*}$ mit abnehmendem Wellenvolumen $\Delta V^{*}$. Diese Beobachtung steht im Gegensatz zu der charakteristischen Welleneigenschaft, daß die Geschwindigkeit mit der Amplitude zunimmt. Die Ursache für diesen Widerspruch ist nicht ein grundsätzlich anderes Verhalten kleiner Wellen, sondern die begrenzte Gültigkeit der verwendeten Skalierung für thermische Plumes. Die Skalierung stößt hier offenbar an ihre Grenzen, da die diffusive Auskühlung bei kleineren Wellen eine größere Rolle spielt. Außerdem verändern sich bei kleinen Volumenflüssen auch die Eigenschaften des Plumekanals durch Auskühlung stark mit der Höhe.

Da in ,starken“ Plumekanälen die thermische Diffusion eine verhältnismäßig geringere Rolle spielt, haben darin aufsteigende Wellen die größte Ähnlichkeit mit den von Olson und Christensen [1986] untersuchten Wellen, so daß für diese Fälle die angewendete Skalierung für dimensionslose Volumina kleiner 11 die konsistentesten Ergebnisse liefert (vergl. Kurven für 120 und $200 \mathrm{ml} / \mathrm{min}$ Volumenfluß in Abbildung 5.1). Für Werte von $\Delta V^{*} \geq 11$ beobachtet man starke Schwankungen in der dimensionslosen Geschwindigkeit $C^{*}$, die vermutlich auf die langgestreckte Form dieser Wellen zurückzuführen sind. Die Wellenlänge ist zum Teil so groß, daß in einem vergleichbar langen Bereich des stationären Kanals die Viskosität um den Faktor 2 mit der Höhe zunimmt. Dadurch trifft der Anfang der Welle auf deutlich viskoseres Material als das Ende, so daß die Physik des Wellenaufstiegs stark von der thermischen Struktur des Kanals verfälscht wird (vergl. Diskussion im folgenden Abschnitt 5.2).

Zusammenfassend läßt sich festhalten, daß die für chemischen Plumes entwickelte Skalierung auch für die Untersuchung von thermischen Plumes und den darin aufsteigenden Wellen geeignet ist, solange die Wellenlängen klein gegenüber den vertikalen Veränderungen des Plumekanals bleiben und der Hintergrundvolumenstrom sowie der Volumeninhalt der Welle so groß ist, daß diffusive Effekte klein bleiben.

\subsection{Temperaturanomalie der Wellen}

Aus geophysikalischer Sicht ist die Untersuchung der durch die Wellen transportierten Temperaturanomalien von großer Bedeutung. Dabei spielt insbesondere die maximale Temperaturerhöhung in der Welle gegenüber dem Plumekanal eine wichtige Rolle. Bei schwachen, vollständig ausgebildeten Plumekanälen, die unterhalb der Lithosphäre so stark ausgekühlt sind, daß keine oder nur geringe Mengen an partiellen Schmelzen gebildet werden, kann eine aufsteigende Welle dafür sorgen, daß die Soliduskurve des Mantelgesteins deutlicher überschritten wird. So können größere Schmelzenmengen entstehen, die einen verstärkten 
Vulkanismus an der Erdoberfläche zur Folge hätten. Periodisch hintereinander aufsteigende Wellen könnten sich dann über Millionen Jahre hinweg als ,pulsierender“ Vulkanismus an der Erdoberfläche bemerkbar machen.

Skaliert man die Höhe (z-Achse) mit der diffusiven Längenskala $Q / \kappa$ (mit $Q$ Plumevolumenfluß, $\kappa$ thermische Diffusivität), so fallen Plumekanäle unterschiedlicher Stärke, wie in Abbildung 5.2 dargestellt, annähernd übereinander. In den folgenden Darstellungen wird die z-Koordinate auf die gleiche Weise skaliert.

Abbildung 5.3 zeigt beispielhaft das Verhältnis zwischen maximaler Wellentemperatur und maximaler Kanaltemperatur im Plumezentrum in Abhängigkeit von der dimensionslosen Aufstiegshöhe $z^{*}$ für 5 verschiedene Hintergrundvolumenflüsse. Für die Wellenerzeugung ist der Volumenfluß in diesem Fall jeweils um den Faktor 10 für 2 Sekunden erhöht worden. Die schwarze und längste Kurve stellt das Temperaturverhältnis beim geringsten Volumenfluß (40 ml/min) dar, die gelbe und kürzeste Kurve das beim stärksten Hintergrundvolumenfluß (120 $\mathrm{ml} / \mathrm{min})$.

Die Form der Kurven ist in allen Fällen sehr ähnlich und durchläuft jeweils ein Maximum (außer bei der gelben Kurve, wo die Höhe des Rechengebietes nicht ausreichte). Die Ursache für das Auftreten eines Maximums liegt darin, daß bei einem schnellen Wellenaufstieg der Temperaturkontrast zwischen Welle und Kanal zunächst immer größer wird. Dadurch erhöht sich der Temperaturgradient, so daß der konduktive Wärmetransport aus der Welle heraus langsam ansteigt. Die zunehmend kleiner werdenden Aufstiegsgeschwindigkeiten der Wel-

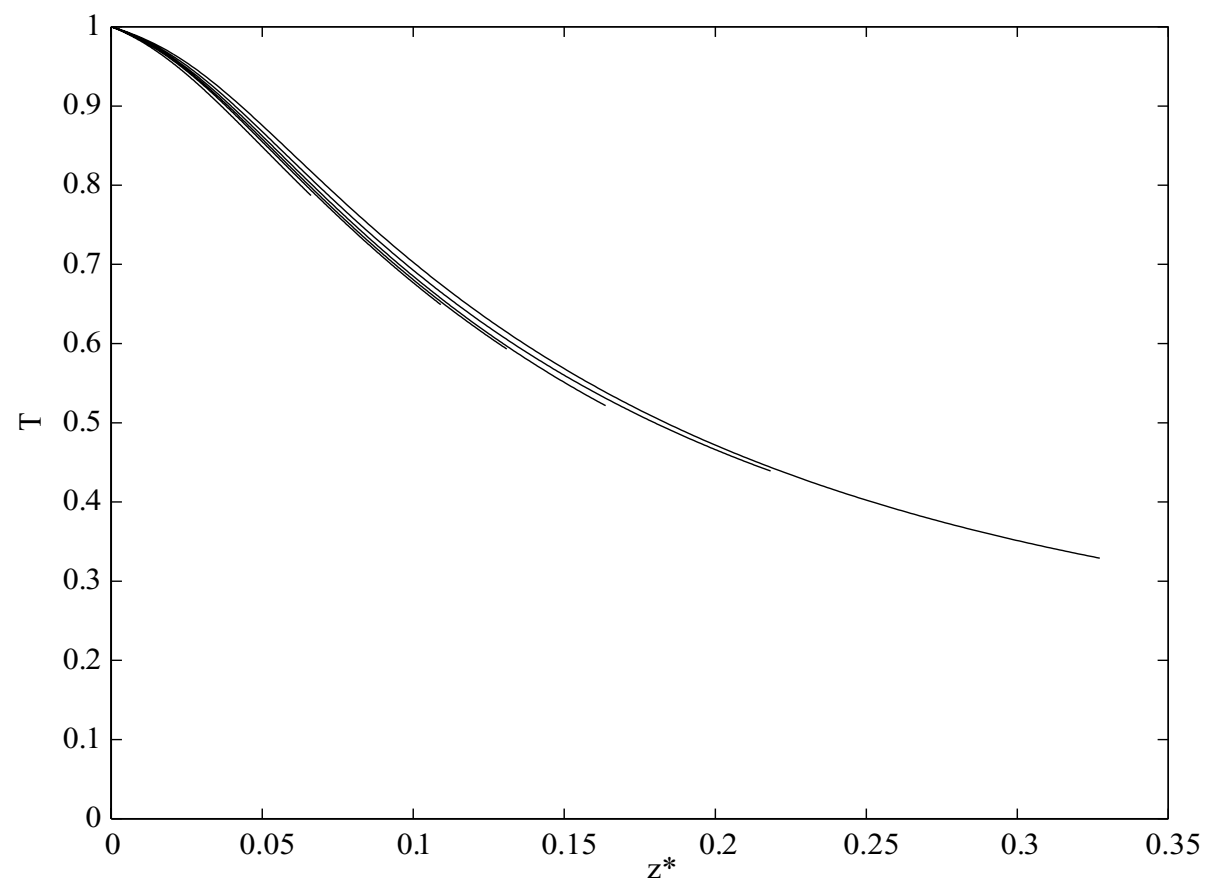

Abbildung 5.2: Zentrumstemperaturen für Plumekanäle mit Hintergrundvolumenflüssen von 40-120 ml/min. Die z-Koordinate ist mit der diffusiven Längenskala $Q / \kappa$ skaliert worden. 


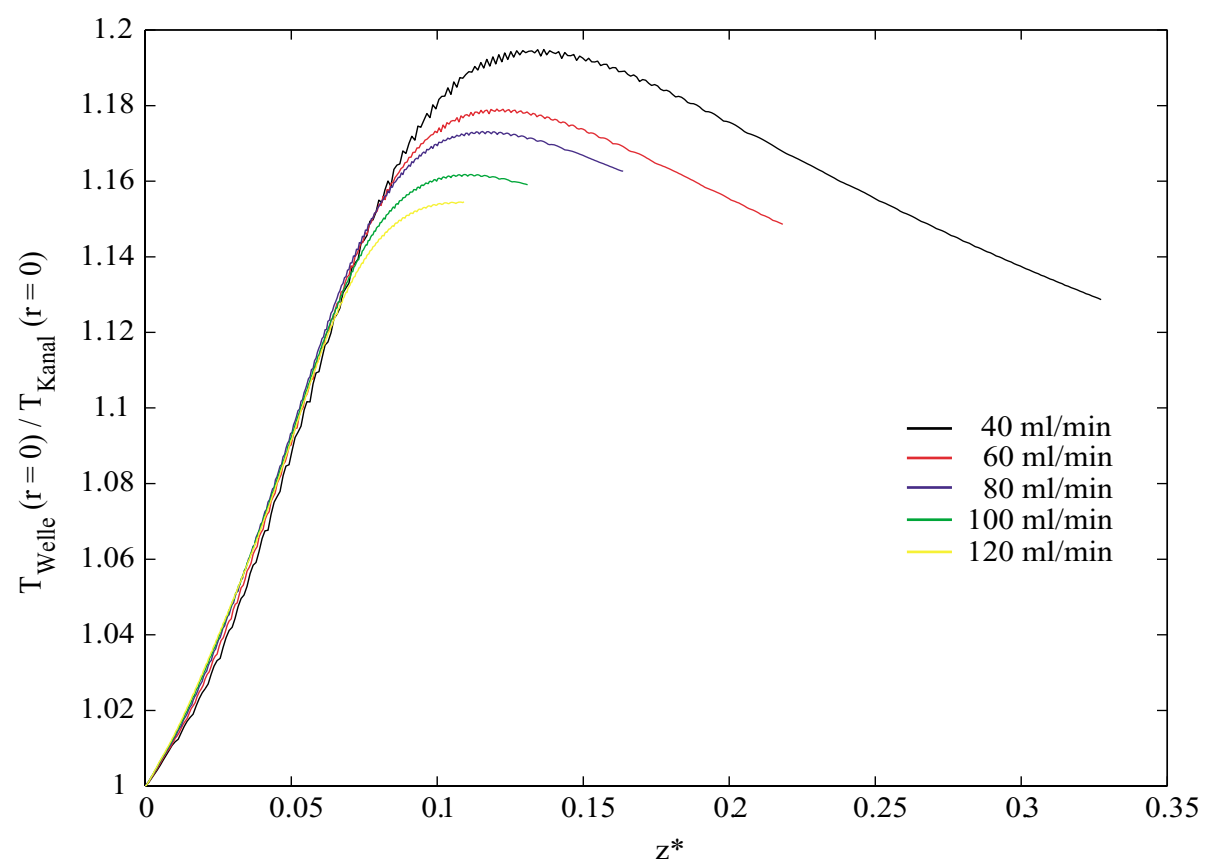

Abbildung 5.3: Verhältnis zwischen maximaler Wellentemperatur und maximaler Kanaltemperatur in Abhängigkeit von der dimensionslosen Aufstiegshöhe $z$ für verschiedene Hintergrundvolumenflüsse zwischen $40 \mathrm{ml} / \mathrm{min}$ (schwarze Kurve) und $120 \mathrm{ml} / \mathrm{min}$ (gelbe Kurve).

len lassen schließlich die diffusive Auskühlung so stark anwachsen, daß das Verhältnis der Temperaturen wieder kleiner wird.

Man erkennt, daß die Welle beim schwächsten Hintergrundvolumenfluß die größte Temperaturerhöhung verursacht. Außerdem verschiebt sich das Maximum der Kurven mit abnehmendem Volumenfluß des Kanals geringfügig zu größeren Aufstiegshöhen hin. Ein ähnliches Bild ergibt sich auch für Wellen, die aus größeren bzw. kleineren Volumenstörungen entstanden sind.

Abbildung 5.4 zeigt in analoger Form die Temperaturverhältnisse für verschiedene Wellen und unterschiedliche Hintergrundvolumenflüsse. Graphen gleicher Farbe gehören zu Wellen mit gleichen Erzeugungsmerkmalen. Da die Welleneigenschaften - wie in Kapitel 4 gezeigt nur von dem zusätzlich injizierten Wellenvolumen abhängen, stellen die in der Legende angegebenen Wellentypen jeweils nur Beispiele für Wellen eines Volumens dar. Kurven für andere Parameterkombinationen, die zu gleichen Wellenvolumina führen würden, sind nicht eingezeichnet, da sie sich nicht von den anderen Kurven unterscheiden ließen. Die Zuordnung zum Hintergrundvolumenfluß ergibt sich analog zu Abbildung 5.3 aus der Länge der dargestellten Graphen.

Das in Abbildung 5.4 dargestellte Ergebnis macht deutlich, daß die durch die Wellen erzeugte Temperaturanomalie systematisch von der Volumenflußerhöhung abhängt. Außerdem beobachtet man für Wellen in schwächeren Plumekanälen größere Temperaturanomalien im 


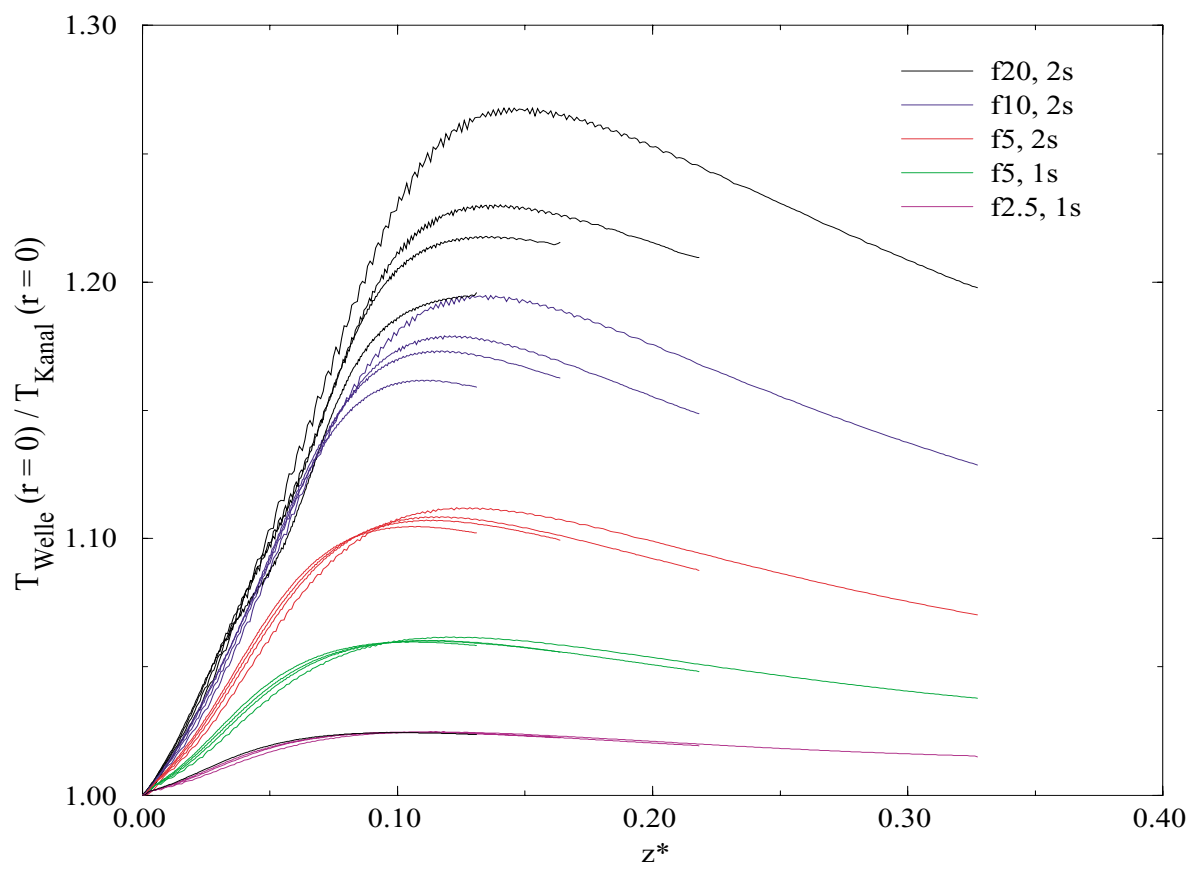

Abbildung 5.4: Verhältnis zwischen maximaler Wellentemperatur und maximaler Kanaltemperatur in Abhängigkeit von der dimensionslosen Aufstiegshöhe $z^{*}$ für verschiedene Hintergrundvolumenflüsse und unterschiedlich große wellenerzeugende Volumenstörungen.

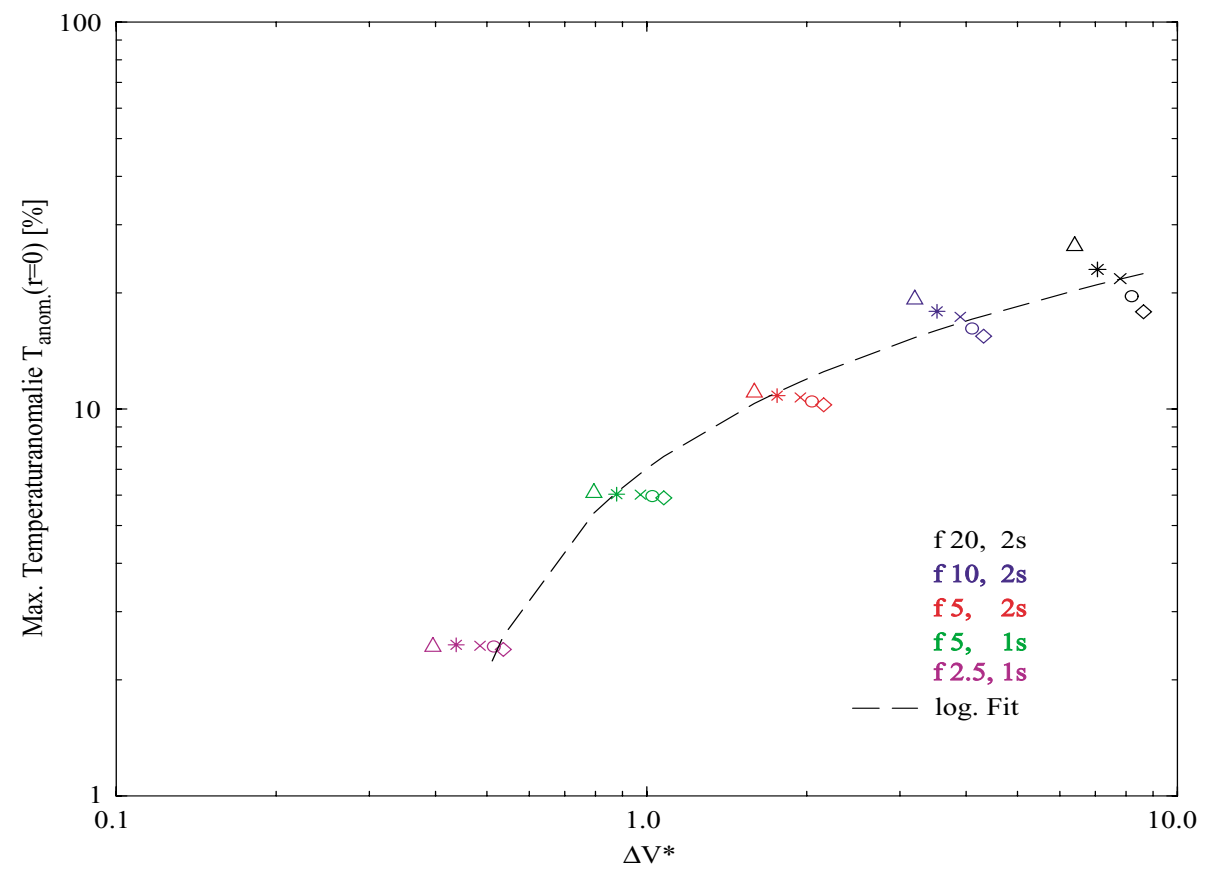

Abbildung 5.5: Maximale prozentuale Temperaturanomalie bei $r=0$ im Vergleich zum stationären Kanal gegenüber dimensionslosem Wellenvolumen $\Delta V^{*}$. Gleiche Symbole stehen für gleiche Hintergrundvolumenflüsse (vergl. Tabelle 5.1). Funktion der empirisch angebrachten Anpassung:

$T_{\text {anom }}=\left(0.07+0.0713 \ln \Delta V^{*}\right) \cdot 100 \%$ 
Vergleich zu den jeweiligen Temperaturen im Kanal.

In Abbildung 5.5 sind in einer Auftragung die aus Abbildung 5.4 bestimmten maximalen prozentualen Temperaturanomalien der Wellen gegenüber den dimensionlosen Wellenvolumina $\Delta V^{*}$ (skaliert gemäß Gleichung A.8 unter Verwendung der Größen am Einströmpunkt) dargestellt. Symbole gleicher Farbe entsprechen dabei Wellen mit gleichen Erzeugungsmerkmalen, während gleiche Symbole zu gleichen Hintergrundvolumenflüssen (genaue Zuordnung s. Tabelle 5.1) gehören. Man erkennt, daß die Skalierung die Symbole nicht exakt auf einen Graphen vereint, sondern innerhalb eines Wellentyps (gleiche Farben) systematische Schwankungen auftreten, die vermutlich auf die bereits angesprochene, stark variierende Wellenlänge zurückzuführen sind. In Tabelle 5.1 sind die vertikalen Ausdehnungen verschiedener Wellen in mittlerer Aufstiegshöhe für unterschiedliche Hintergrundvolumenflüsse aufgeführt. Da die Temperaturanomalien der Wellen aufgrund der in Kapitel 4.2.1 diskutierten langgezogenen Strukturen hinter den Wellen kein zuverlässiges Kriterium für die vertikalen Ausdehnungen liefern können, werden hier Bereiche 5\%-iger Geschwindigkeitserhöhungen gegenüber den stationären Geschwindigkeitsfeldern mit den Wellen assoziiert.

Tabelle 5.1 macht deutlich, daß die dimensionslosen Wellenlängen stark mit dem Wellenvolumen zunehmen. Außerdem zeigen die Tabellenzeilen eine Zunahme der Wellenlänge mit abnehmendem Hintergrundfluß.

Im Zentrum stationärer Plumekanäle verdoppelt sich die Viskosität in vertikaler Richtung bei einer dimensionslosen Höhenzunahme von 0.055. Tabelle 5.1 zeigt, daß dieser Wert in-

Tabelle 5.1: Skalierte Wellenlängen

\begin{tabular}{c|cccccc}
\hline Wellen- & Plumefluß: & $120 \mathrm{ml} / \mathrm{min}$ & $100 \mathrm{ml} / \mathrm{min}$ & $80 \mathrm{ml} / \mathrm{min}$ & $60 \mathrm{ml} / \mathrm{min}$ & $40 \mathrm{ml} / \mathrm{min}$ \\
typ & Symbol: & $\diamond$ & $\circ$ & $\times$ & $*$ & $\triangle$ \\
\hline f2.5, $1 \mathrm{~s}$ & & 0.0079 & 0.0086 & 0.0092 & 0.0092 & 0.0094 \\
f5, 1s & & 0.015 & 0.016 & 0.017 & 0.020 & 0.025 \\
f5, $2 \mathrm{~s}$ & & 0.021 & 0.022 & 0.024 & 0.025 & 0.032 \\
$\mathrm{f} 10,2 \mathrm{~s}$ & & 0.039 & 0.044 & 0.049 & 0.049 & 0.049 \\
$\mathrm{f} 20,2 \mathrm{~s}$ & & 0.051 & 0.06 & 0.07 & 0.079 & 0.081 \\
\hline
\end{tabular}

Anmerkung: Tabelliert ist die dimensionslose vertikale Ausdehnung (skaliert mit $Q / \kappa)$ verschiedener Wellen in mittlerer Zylinderhöhe für verschiedene Hintergrundvolumenflüsse. Die Parameterkombinationen der hier angegebenen Wellentypen sind in dieser Form in den Modellierungen verwendet worden. Sie sind an dieser Stelle als Beispiele für Parameterkombinationen zu verstehen, die auf gleiche Wellenvolumina führen würden. Das Wellenvolumen nimmt in der Tabelle von oben nach unten zu. Für die Lokalisierung der Welle ist der Bereich einer 5\%-igen Erhöhung der Vertikalgeschwindigkeit im Vergleich zur Kanalgeschwindigkeit auf der Zylinderachse herangezogen worden. Die skalierte Länge bei, der sich die Plumeviskosität des Kanals in vertikaler Richtung um den Faktor 2 ändert, beträgt 0.055. Die zu dem jeweiligen Hintergrundvolumenfluß gehörenden Symbole beziehen sich auf Abbildung 5.5. 
nerhalb eines Wellentyps insbesondere bei kleinen Plumeflüssen erreicht (teilweise sogar überschritten) werden kann, während die Wellenlängen bei größeren Plumeflüssen zum Teil weit davon entfernt sind. Daher ergeben sich innerhalb eines Wellentyps stark voneinander abweichende Hintergrundbedingungen, was als Hauptursache dafür angesehen wird, daß die Symbole eines Wellentyps in Abbildung 5.5 nicht genau aufeinander fallen. Um dennoch möglichst einfache Zusammenhänge zu finden, die sich geophysikalisch interpretieren lassen, wurden mittlere Werte der dargestellten Ergebnisse bestmöglich durch eine empirische logarithmische Funktion angepaßt. Diese Anpassung wird in Abschnitt 5.4 bei der Skalierung auf die Plumes der Erde verwendet.

\subsection{Einfluß einer tiefenabhängigen Viskosität}

Čadek und Fleitout [1999] finden durch Anpassung von Geoiddaten an ein geschichtetes Erdmodell mit vorgegebenen Plattengeschwindigkeiten eine Tiefenzunahme der Viskosität vom oberen zum unteren Erdmantel um etwa 2 Größenordnungen. Andere Studien verwenden Daten von nacheiszeitlichen Hebungsraten (z.B.: Lambeck et al.[1998] und Kaufmann und Lambeck [2000]) und kommen auf ähnliche Viskositätsvariationen. Für die hier durchgeführten Rechnungen mit tiefenabhängiger Viskosität ist die Konstante $c_{v i s c}$ in Gleichung 3.24 auf den Wert 2 gesetzt worden, so daß die Viskosität bei konstanter Temperatur über die Zylinderhöhe um zwei Größenordnungen von oben nach unten exponentiell zunimmt. Abbildung 5.6 zeigt die Zentrumstemperaturen stationärer Plumekanäle bei tiefenabhängiger

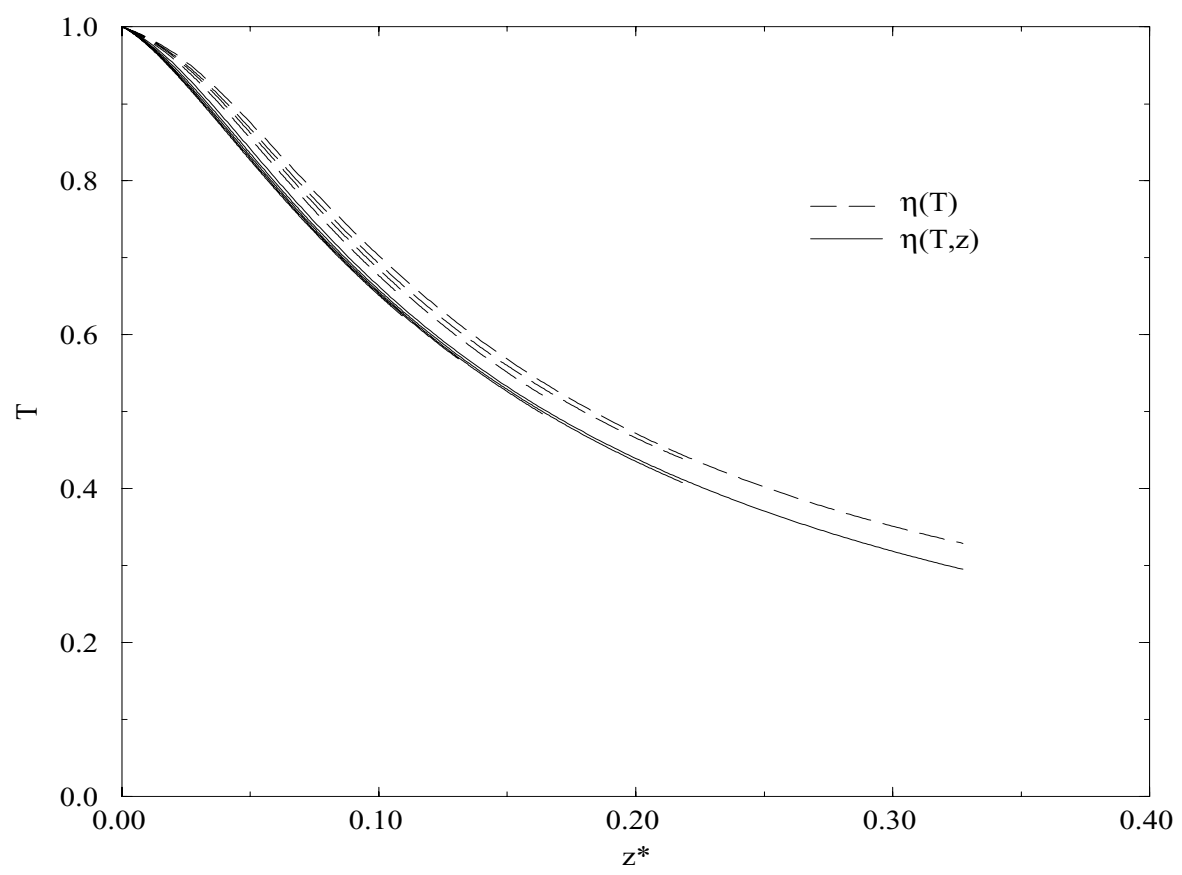

Abbildung 5.6: Zentrumstemperaturen für Plumekanäle mit und ohne tiefenabhängige Viskosität für verschiedene Hintergrundvolumenflüsse von 40-120 ml/min. Die z-Koordinate wurde mit der diffusiven Längenskala $Q / \kappa$ skaliert. 
Viskosität im Vergleich zu rein thermischer Viskosität. Man erkennt, daß die Kanaltemperaturen im Fall der tiefenabhängigen Viskosität geringfügig kleiner sind als bei Plumes mit ausschließlich thermischer Viskositätsabhängigkeit.

Deutlicher sind die Unterschiede in den Vertikalgeschwindigkeiten. Abbildung 5.7 zeigt, daß bei Rechnungen mit tiefenabhängiger Viskosität die Vertikalgeschwindigkeiten im unteren Bereich des Zylinders stark verringert sind. Dadurch kommt es zu einer Verbreiterung des zu Beginn aufsteigenden Plumekopfes (Abbildung 5.8 a) und des stationären Kanals (Abbildung $5.8 \mathrm{c}$ ).

Im oberen Bereich des Zylinders, in dem die Viskosität erniedrigt ist, nehmen die Vertikalgeschwindigkeiten trotz abnehmender Temperaturanomalie stark zu. Als Folge dessen verringert sich die Ausdehnung des Plumekopfes und des Plumekanals mit zunehmender Aufstiegshöhe (Abbildung 5.8 b bzw. c). Die Beobachtung eines mit der Höhe schmaler werdenden Plumekanals ist konsistent zu Ergebnissen anderer Studien (z.B. Hauri et al., [1994]).

Aufsteigende Wellen verhalten sich analog zu dieser Beobachtung. Sie haben im Bereich der erhöhten Viskosität stark verlangsamte, in größeren Zylinderhöhen aber stark ansteigende Geschwindigkeiten im Vergleich zu Wellen in Modellen mit rein thermischer Viskosität. Man beobachtet dabei auch, daß die Welle im Modell mit tiefenabhängiger Viskosität eine etwas langgestrecktere Form aufweist, was durch quantitative Betrachtungen der Geschwindigkeitsanomalien bestätigt wird. Abbildung 5.9 zeigt den direkten Vergleich von Differenz-

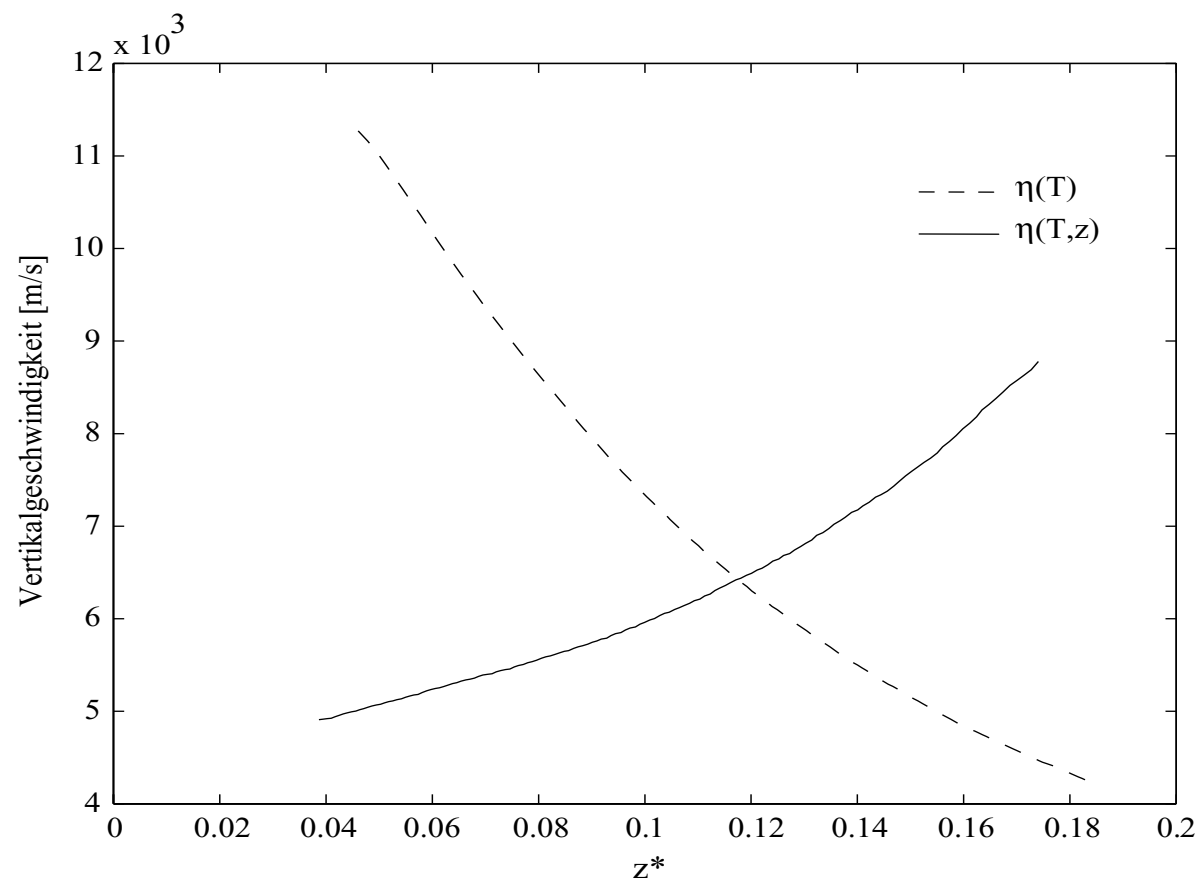

Abbildung 5.7: Beispiel für die Vertikalgeschwindigkeit im Zentrum von einem Plumekanal mit rein thermischer und einem Plumekanal mit zusätzlich tiefenabhängiger Viskosität. 


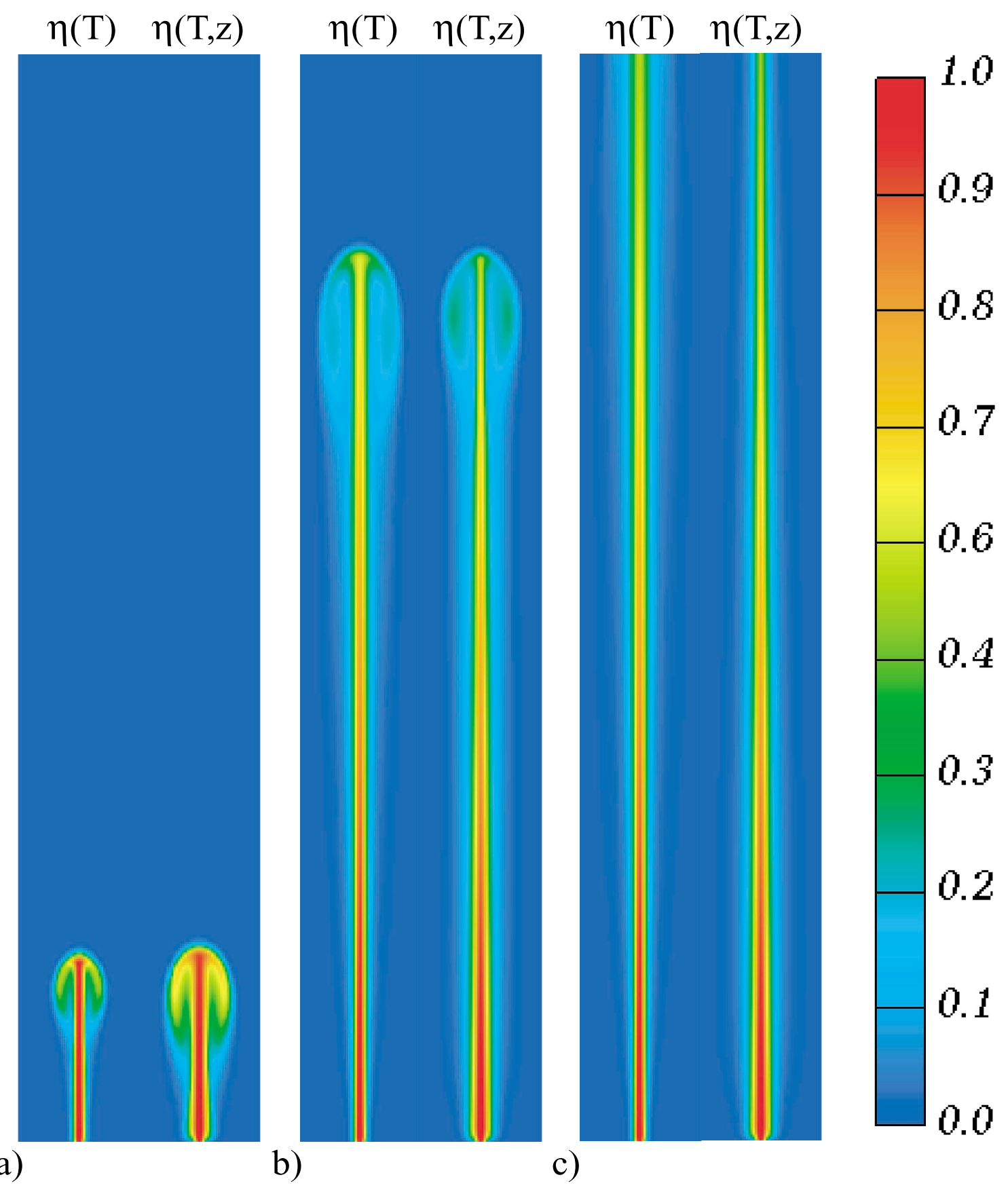

Abbildung 5.8: Vergleich eines Plumeaufstiegs für rein thermische Viskosität $\eta(T)$ und zusätzlich tiefenabhängiger Viskosität $\eta(T, z)$. Dargestellt sind Temperaturfelder einer Plumeströmung mit konstantem Volumenfluß zu jeweils zwei verschiedenen Zeitpunkten ( $a, b)$ und nach Erreichen des stationären Zustands (c). Dargestellte Zylindergröße $0.2 \times 2 \mathrm{~m}$. 


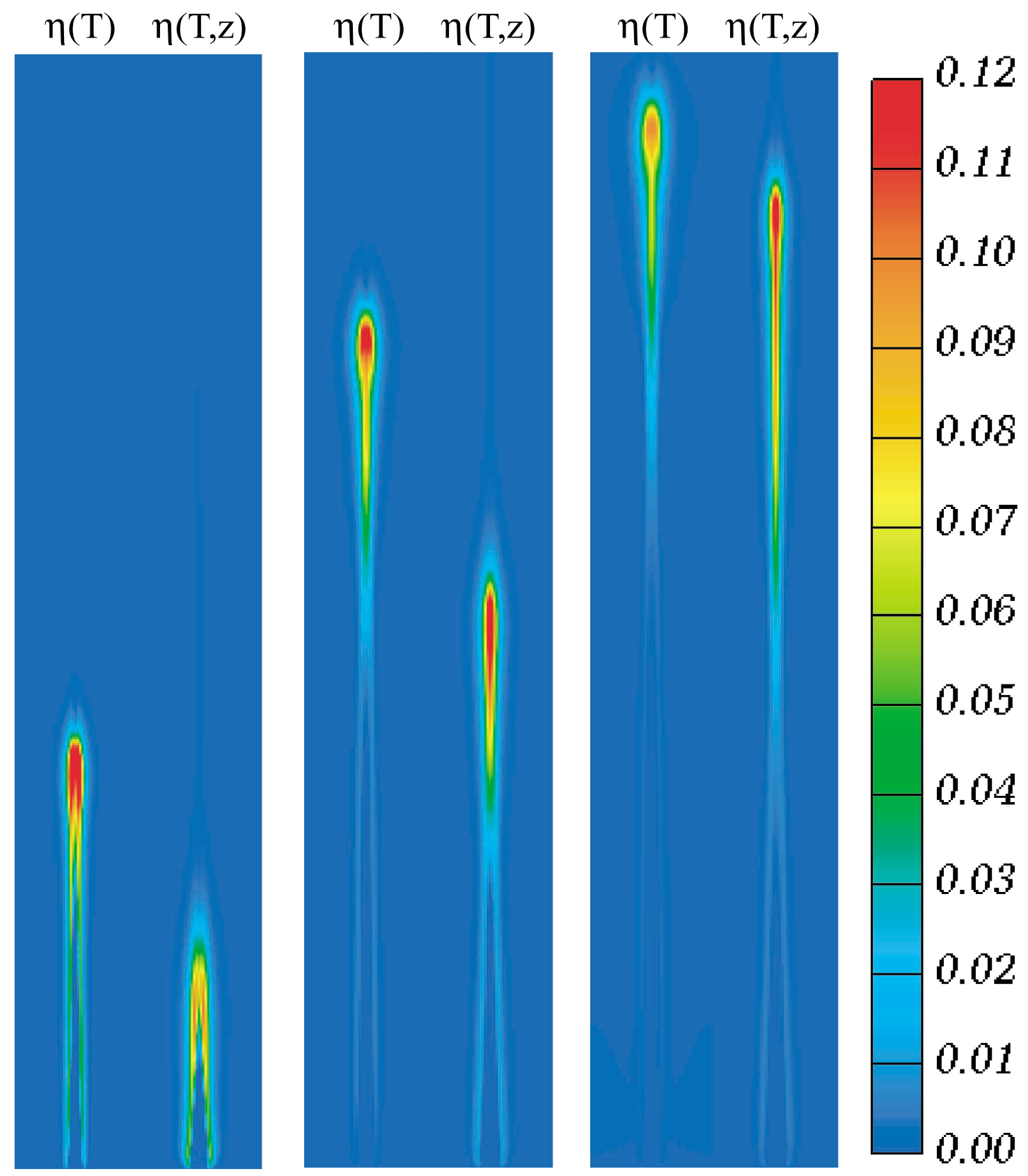

a)

b)

c)

Abbildung 5.9: Vergleich zwischen einem Wellenaufstieg für rein thermische Viskosität $\eta(T)$ und einem mit zusätzlich tiefenabhängiger Viskosität $\eta(T, z)$. Dargestellt sind die Differenztemperaturfelder zu den jeweiligen stationären Plumekanälen. Auf ein (theoretisches) Laborexperiment bezogen, betragen in diesen Rechnungen die Hintergrundvolumenflüsse $60 \mathrm{ml} / \mathrm{min}$ mit Temperaturanomalien von $75 \mathrm{~K}$. Die Volumenstörungen wurden jeweils durch zwei Sekunden (bezogen auf die experimentelle Zeitskala) andauernde Erhöhungen des Volumenflusses um den Faktor 20 erzeugt. Verstrichene Zeit seit Wellenerzeugung, bezogen auf die experimentelle Zeitskala: a) $44 \mathrm{~s}$; b) $141 \mathrm{~s}$; c) $217 \mathrm{~s}$. Dargestellte Zylindergröße $0.2 \times 2 \mathrm{~m}$. 
temperaturfeldern einer Wellenausbreitung bei rein thermischer und bei zusätzlich tiefenabhängiger Viskosität.

Abbildung 5.10 zeigt beispielhaft den Einfluß der tiefenabhängigen Viskosität auf die Maximaltemperaturen in einer Welle. Man erkennt, daß die tiefenabhängige Viskosität keinen großen Einfluß auf die Wellentemperatur hat. Da sowohl Welle als auch Kanal bei tiefenabhängiger Viskosität geringfügig kälter sind, ergibt sich auch für die Temperaturanomalie der Wellen kein nennenswerter Effekt. Auch für Wellen mit anderen Erzeugungsparametern bzw. Volumen zeigt sich im wesentlichen das gleiche Bild. Der Einfluß tiefenabhängiger Viskosität auf die Temperaturanomalie einer Welle kann darum näherungsweise vernachlässigt werden. Aus diesem Grund werden im nachfolgenden Abschnitt die Parameterstudienergebnisse des einfacheren Modells mit rein temperaturabhängiger Viskosität auf die Erde skaliert.

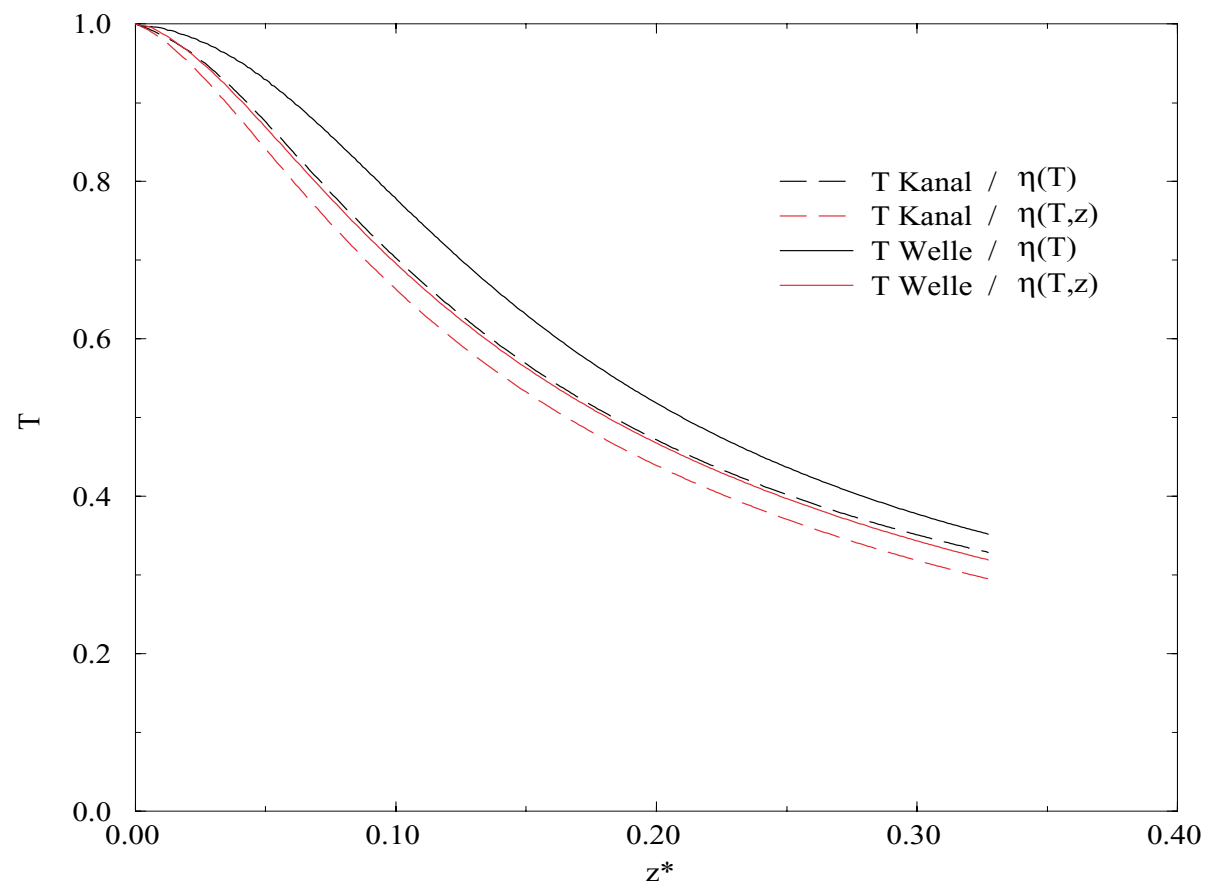

Abbildung 5.10: Beispielhafter Vergleich der Maximaltemperaturen in aufsteigenden Wellen mit gleichen Parametern, gerechnet mit und ohne tiefenabhängige Viskosität im Vergleich zu den Zentrumstemperaturen der dazugehörigen Kanäle.

\subsection{Skalierung auf die Mantelplumes der Erde}

Um beurteilen zu können, welche Rolle die in Experiment und Numerik beobachteten Wellen in den Mantelplumes der Erde spielen könnten, werden die in den Abschnitten 5.1 und 5.2 zusammengefaßten Ergebnisse auf die Erde reskaliert. Dazu werden die in Anhang A.3 aufgeführten Skalen und die empirisch gefundenen Zusammenhänge zwischen dimensionslosem Wellenvolumen $\Delta V^{*}$ und Ausbreitungsgeschwindigkeit $C^{*}$ (vergl. Abbildung 5.1), so- 
Tabelle 5.2: Die für die Reskalierung verwendeten Parameter

\begin{tabular}{clcc}
\hline Parameter & Bedeutung & Wert & Einheit \\
\hline$T_{0}$ & Mittlere Temperatur des Erdmantels & 1573 & $\mathrm{~K}$ \\
$\Delta T$ & Temperaturanomalie des Plumes & 300 & $\mathrm{~K}$ \\
$\alpha$ & Therm. Ausdehnungskoeffizient & $3.5 \times 10^{-5}$ & $\mathrm{~K}^{-1}$ \\
$\eta_{M}$ & Mittlere Viskosität des Mantels & $5 \times 10^{21}$ & Pas \\
$\eta_{L}$ & Viskosität des Plumematerials & $1 \times 10^{18}$ & $\mathrm{Pas}$ \\
$\kappa$ & Thermische Diffusivität & $10^{-6}$ & $\mathrm{~m}^{2} / \mathrm{s}$ \\
$g$ & Schwerebeschleunigung & 9.81 & $\mathrm{~m} / \mathrm{s}^{2}$ \\
$\rho_{0}$ & Mittlere Dichte des Mantelgesteins & 3300 & $\mathrm{~kg} / \mathrm{m}^{3}$ \\
$Q_{0}$ & Volumenfluß des Plumes & $29-100$ & $\mathrm{~m}^{3} / \mathrm{s}$ \\
\hline
\end{tabular}

wie Volumen $\Delta V^{*}$ und Temperaturanomalie $T_{\text {anom }}$ (vergl. Abbildung 5.5) verwendet.

Die für die Erde angenommenen Parameter sind in Tabelle 5.2 aufgeführt. Die Werte des Plumeflusses $Q_{0}$ decken einen Bereich ab, dessen Untergrenze aus dem von Albers und Christensen [1996] angegeben minimalen Auftriebsfluß von $B=1000 \mathrm{~kg} / \mathrm{s}$ für Plumes, die von der Kern-Mantelgrenze stammen, bestimmt wurde. Die Studie besagt, daß Plumes, die aus 2900 km Tiefe aufsteigen, diesen Mindestauftriebsfluß haben müssen, um unter der Lithosphäre noch Schmelzen erzeugen zu können. Der Volumenfluß $Q_{0}$ ist über

$$
Q_{0} \equiv \frac{B}{\rho_{0} \alpha \Delta T}
$$

mit dem Auftriebsfluß $B$ verknüpft (Ribe et al., [1995]), so daß sich mit den hier für $\rho_{0}, \alpha$ und $\Delta T$ angenommenen Werten (vergl. Tabelle 5.2) ein Mindestwert für $Q_{0}$ von $29 \mathrm{~m}^{3} / \mathrm{s}$ ergibt. Da auf der Erde der Hawaii-Plume den größten Auftriebsfluß besitzt, wird dieser für die Wahl der Obergrenze von $Q_{0}$ verwendet. Ribe und Christensen [1999] bestimmen in einem 3DKonvektionsmodell unter Berücksichtigung von Schmelzenproduktion eine Auftriebsflußspanne zwischen $2200 \mathrm{~kg} / \mathrm{s} \leq B \leq 3500 \mathrm{~kg} / \mathrm{s}$ in der ihr Modell mit für den Hawaii-Plume beobachteten Schmelzraten, sowie Höhe und Breite der topographischen Lithosphärenplattenaufwölbung konsistent ist. Dabei entspricht ein Auftriebsfluß von $3500 \mathrm{~kg} / \mathrm{s}$ gerade einem Volumenfluß von etwa $100 \mathrm{~m}^{3} / \mathrm{s}$.

In Tabelle 5.3 sind die Ergebnisse der Reskalierung für einen systematisch variierten Volumenfluß $Q_{0}$ angegeben. Da es, wie im Zusammenhang mit Abbildung 4.10 diskutiert, nur auf den Volumeninhalt der Welle bzw. das zusätzlich vom Kanal aufgenommene Volumen ankommt, können die Werte der zweiten und dritten Spalte in Tabelle 5.3 im gewissen Rahmen variiert werden, solange sich die absolute Menge des Wellenmaterials (vierte Spalte) dabei nicht ändert. Hier sind in der zweiten und dritten Spalte geophysikalisch möglichst plausible Parameterkombinationen angegeben worden. Die Wellenlängen (fünfte Spalte) sind aus den in Tabelle 5.1 angegebenen Werten abgeschätzt worden. Die Ergebnisse für die Geschwindigkeit und die Temperaturanomalie im Vergleich zum Plumekanal (sechste und siebte Spalte) wurden aus den empirischen Anpassungen der Parameterstudien bestimmt. Die letzte 
Tabelle 5.3: Ergebnisse der Reskalierung

\begin{tabular}{cccccccc}
\hline $\begin{array}{c}\text { Plumefluß } \\
{\left[\mathrm{m}^{3} / \mathrm{s}\right]}\end{array}$ & $\begin{array}{c}\text { Faktor } \\
-\end{array}$ & $\begin{array}{c}\text { tStörung } \\
{[\mathrm{Ma}]}\end{array}$ & $\begin{array}{c}\text { Wellenvol. } \\
{\left[\mathrm{km}^{3}\right]}\end{array}$ & $\begin{array}{c}\text { Wellenlänge } \\
{[\mathrm{km}]}\end{array}$ & $\begin{array}{c}\text { Geschw. } \\
{[\mathrm{m} / \mathrm{a}]}\end{array}$ & $\begin{array}{c}T_{\text {anom. }} \\
{[\%]}\end{array}$ & $\begin{array}{c}\text { Zeit } \\
{[\mathrm{Ma}]}\end{array}$ \\
\hline 29 & 2 & 0.32 & $5.9 \times 10^{5}$ & 400 & 0.87 & 5 & 0.5 \\
29 & 2 & 0.43 & $7.9 \times 10^{5}$ & 580 & 0.88 & 7 & 0.7 \\
29 & 1.5 & 1.15 & $1.6 \times 10^{6}$ & 810 & 0.91 & 12 & 0.9 \\
40 & 1.5 & 0.40 & $7.5 \times 10^{5}$ & 560 & 1.02 & 5 & 0.5 \\
40 & 1.5 & 0.53 & $1.0 \times 10^{6}$ & 800 & 1.03 & 7 & 0.8 \\
40 & 1.5 & 1.06 & $2.0 \times 10^{6}$ & 1100 & 1.07 & 12 & 1.0 \\
55 & 1.5 & 0.37 & $9.6 \times 10^{5}$ & 770 & 1.20 & 5 & 0.6 \\
55 & 1.5 & 0.49 & $1.3 \times 10^{6}$ & 1100 & 1.21 & 7 & 0.9 \\
63 & 1.5 & 0.36 & $1.1 \times 10^{6}$ & 890 & 1.28 & 5 & 0.7 \\
63 & 1.5 & 0.47 & $1.4 \times 10^{6}$ & 1260 & 1.29 & 7 & 1.0 \\
70 & 1.5 & 0.23 & $7.7 \times 10^{5}$ & 600 & 1.34 & 2 & 0.5 \\
70 & 1.5 & 0.35 & $1.1 \times 10^{6}$ & 980 & 1.35 & 5 & 0.7 \\
70 & 1.5 & 0.46 & $1.5 \times 10^{6}$ & 1400 & 1.36 & 7 & 1.0 \\
85 & 1.5 & 0.22 & $8.9 \times 10^{5}$ & 730 & 1.47 & 2 & 0.5 \\
85 & 1.5 & 0.33 & $1.3 \times 10^{6}$ & 1200 & 1.49 & 5 & 0.8 \\
100 & 1.5 & 0.21 & $1.0 \times 10^{6}$ & 860 & 1.60 & 2 & 0.5 \\
\hline
\end{tabular}

Anmerkung: Tabelliert sind die Ergebnisse der Reskalierung für unterschiedliche Werte von Q. Dabei geben die zweite und dritte Spalte den Faktor und die zeitliche Dauer ( ${ }_{\text {ttörung }}$ ) der Volumenflußerhöhung an. In den nachfolgenden Spalten folgt das absolute Wellenvolumen in $\mathrm{km}^{3}$, sowie die Wellenlänge, die Aufstiegsgeschwindigkeit, die durch die Welle verursachte prozentuale Erhöhung der Plumekanaltemperaturanomalie im Vergleich zur Manteladiabate und die abgeschätzte Zeit während der die Welle einen potentiellen Einfluß auf die Schmelzenproduktion unter der Lithosphäre nehmen könnte.

Spalte gibt die Zeit an, die die Welle benötigt, um eine Wegstrecke entsprechend ihrer eigenen Länge zurückzulegen. Diese Zeit dient als Abschätzung für die Dauer einer möglichen Erhöhung der Schmelzenproduktion.

Für die Berechnung der in Tabelle 5.3 dargestellten Ergebnisse sind Wellen ausgewählt worden, die Temperaturanomalien zwischen 2-12\% gegenüber der Kanaltemperatur erzeugen. Dabei sind für Plumes mit kleinen Hintergrundvolumenflüssen eher größere Störungen angenommen worden als bei Plumes mit großen Flüssen. Der Grund dafür liegt in der stark mit dem Hintergrundvolumenfluß anwachsenden Wellenlänge, wodurch zum Teil Werte von deutlich mehr als $1000 \mathrm{~km}$ entstehen. Derartig langgestreckte Wellen sind in einem $2900 \mathrm{~km}$ tiefen Erdmantel eher unwahrscheinlich.

Im nachfolgenden Kapitel werden die geophysikalischen Resultate dieser Arbeit zusammenfassend diskutiert und anhand von Beispielen die geophysikalische Relevanz der hier dargestellten Ergebnisse aufgezeigt. 


\section{Kapitel 6}

\section{Diskussion}

Die Ergebnisse der Laborexperimente und der systematischen numerischen Parameterstudien haben gezeigt, daß sich Volumenstörungen in thermischen Plumekanälen wellenartig fortbewegen können. Da die Aufstiegsgeschwindigkeiten von den Wellenamplituden abhängen, steigen Wellen mit großem Volumen schneller auf als Wellen mit kleinem Volumen.

Die Tatsache, daß eine Welle, wie in Kapitel 4 gezeigt, eine bevorzugte Form und Ausbreitungsgeschwindigkeit hat, die nicht von der Dauer bzw. der Intensität der Volumenflußerhöhung abhängt, ist aus physikalischer Sicht von großer Bedeutung. Aufgrund dieser Beobachtung kann man davon ausgehen, daß die Wellenerzeugung nicht besonders ,,anspruchsvoll“ ist. Dadurch wird es wahrscheinlicher, daß durch Fluktuationen in der thermischen Grenzschicht - an der der Plume entsteht - Wellen hervorgerufen werden können. Dabei kann man sich vorstellen, daß Instabilitäten die in der Grenzschicht nicht allzuweit von einem bestehenden Plumekanal auftreten, zunächst zu diesem hinwandern und dann vom Kanal aufgenommen werden.

Die in Tabelle 5.3 angegebenen Werte für eine Volumenflußerhöhung um den Faktor 1.5-2, sowie die Zeiten des erhöhten Volumenflusses in der Größenordnung von mehreren hunderttausend Jahren erscheinen in diesem Zusammenhang plausibel. Auch das dadurch entstehende zusätzliche Wellenvolumen in der Größenordnung eines Würfels mit der Kantenlänge von $100 \times 100 \times 100 \mathrm{~km}$ verträgt sich gut mit der allgemeinen Vorstellung, daß Plumes Durchmesser in der Größenordung von $100 \mathrm{~km}$ besitzen.

Kritischer sind die in Tabelle 5.3 angegebenen Wellenlängen, da diese zum Teil so groß werden, daß die Vorstellung einer frei im Kanal aufsteigenden Welle unrealistisch wird. Bei einem Erdmantel von $2900 \mathrm{~km}$ Tiefe mit Phasengrenzen in $660 \mathrm{~km}$ und $410 \mathrm{~km}$ Tiefe, wo insbesondere im Fall der 660 km-Diskontinuität ein sehr starker Einfluß auf aufsteigende Plumes angenommen werden kann (Christensen, [1995]), ist bei Wellenlängen von $1000 \mathrm{~km}$ oder mehr nicht mehr mit dem in dieser Arbeit beobachteten Wellenverhalten zu rechnen.

Betrachtet man als Beispiel für einen starken Plume den Hawaii-Plume und nimmt als Auftriebsfluß die Obergrenze des durch Ribe und Christensen [1999] angegebenen Auftriebsflußintervalls von $B=3500 \mathrm{~kg} / \mathrm{s}$ (entspricht $Q_{0}=100 \mathrm{~m}^{3} / \mathrm{s}$ ) an, so erhält man nach Tabelle 5.3 selbst für eine sehr kleine Störung schon eine Wellenlänge von etwa 860 km Län- 
ge. Eine derartig kleine Störung würde eine etwa 2\%-ige Temperaturerhöhung im Vergleich zur Kanaltemperatur verursachen.

In Abbildung 6.1 ist die Schmelzkurve für trockenen Peridotit als Funktion des Drucks bzw. der Tiefe nach McKenzie und Bickle [1988] aufgetragen. Da es beim Mantelgestein keinen scharfen Übergang zwischen fester und flüssiger Phase gibt, sind in der Darstellung verschiedene Kurven mit unterschiedlichen Schmelzraten zwischen der Solidus- und der Liquiduskurve eingezeichnet. Mit Hilfe dieser Darstellung läßt sich abschätzen, wie groß die Temperaturanomalie eines Plumes unterhalb der Lithosphäre sein muß, damit partielle Schmelzen entstehen können. Dabei nimmt man an, daß die Unterseite der Lithosphäre den Aufstieg des Plumes stoppt, so daß in dieser Tiefe der Solidus überschritten sein muß, damit Schmelzen entstehen können.

Bei einer für Hawaii angenommenen Lithosphärenmächtigkeit in der Größenordnung von $80 \mathrm{~km}$ und einer potentiellen Temperatur des Plumes von $1600^{\circ} \mathrm{C}$ (Ribe und Christensen, [1999]), würde eine 2\%-ige Erhöhung der Kanaltemperatur (entspricht nur etwa 5 K) den

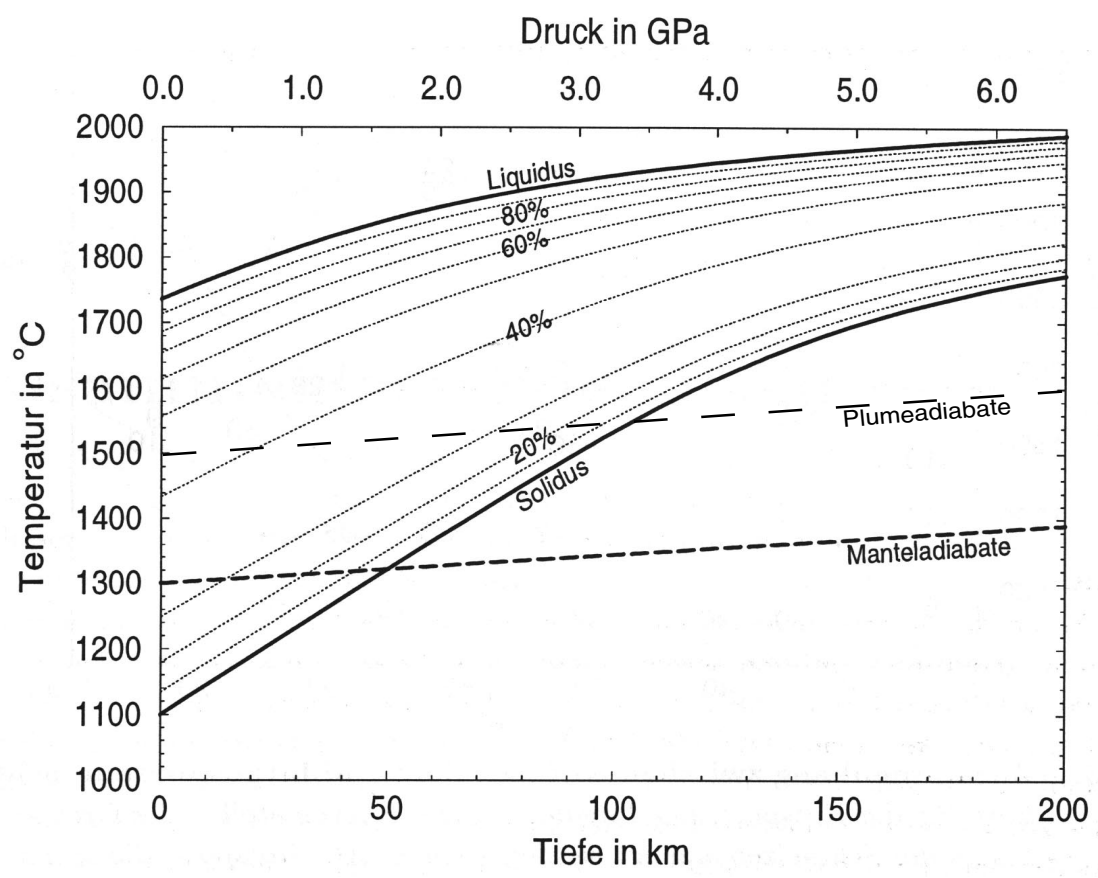

Abbildung 6.1: Schmelzkurve für trockenen Peridotit nach McKenzie und Bickle [1988]. Zwischen der Solidus- und der Liquiduskurve geben die gepunktet dargestellten Kurven den Grad der Aufschmelzung an. Die Manteladiabate mit einer potentiellen Temperatur (an die Erdoberfläche extrapolierte Temperatur der Manteladiabate) von $1300^{\circ} \mathrm{C}$ ist mit dem in Tabelle 5.2 angegebenen thermischen Ausdehnungskoeffizienten $\alpha=3.5 \times 10^{-5} \mathrm{~K}^{-1}$ bestimmt worden. Beispielhaft ist eine Adiabate für einen Plume mit einer potentiellen Temperatur von $1500^{\circ} \mathrm{C}$ eingezeichnet. (Modifizierte Darstellung aus Albers [1995].) 
Grad der Aufschmelzung von etwa 35\% praktisch nicht erhöhen.

Geht man von dem nach Ribe und Christensen [1999] niedrigst möglichen Auftriebsfluß von $B=2200 \mathrm{~kg} / \mathrm{s}$ (entspricht $Q_{0}=63 \mathrm{~m}^{3} / \mathrm{s}$ ) für den Hawaii-Plume aus, so sind Wellen denkbar, deren Längen zwar auch im Bereich von $900 \mathrm{~km}$ lägen, die aber in der Lage wären, 5\%-ige Temperaturanomalien gegenüber dem Kanal zu erzeugen (vergl. Tabelle 5.3). Nach Abbildung 6.1 würde dies bei sonst gleichen Parametern die Aufschmelzrate von 35\% auch nur um wenige Prozentpunkte erhöhen.

Derartig kleine Änderungen im Aufschmelzungsgrad des Gesteins lassen es unwahrscheinlich erscheinen, daß man zeitliche Variationen der Aktivität des Hawaii-Hotspots mit aufsteigenden Wellen in Verbindung bringen kann. Es ist zwar nicht auszuschließen, daß eine Welle die vulkanische Aktivität verstärken könnte, aber der direkte Zusammenhang wäre nur schwer nachzuweisen. Vermutlich würden lokale Einflüsse, die entweder die Schmelzenproduktion erhöhen oder deren Transport erleichtern könnten (Topographie der Lithosphärenunterseite, lokal erhöhter Wassergehalt der Mantelminerale, lokale Bruchzonen o.ä.) die Effekte einer aufsteigenden Welle deutlich übersteigen. Hinzu kommt, daß bei Rechnungen mit tiefenabhängiger Viskosität die Länge der Wellen tendenziell weiter zunimmt, so daß Wellenlängen von mehr als 1000 km erreicht werden können. Durch Wechselwirkungen dieser Wellen mit kleinskaligeren Strukturen, wie Phasengrenzen, könnte die Physik des Wellenaufstiegs so stark beeinflußt werden, daß ein Vergleich mit den in den Modellen beobachteten Wellen unter Umständen nicht mehr gerechtfertigt wäre.

Daher muß man feststellen, daß aufsteigende Wellen in der Form, wie sie in Experiment und Numerik zu beobachten sind, im Hawaii-Plume vermutlich nicht existieren bzw. eine zu vernachlässigende Rolle in der Schmelzenproduktion spielen.

Bei der Betrachtung von schwächeren Plumes wird als Untergrenze ein Mindestvolumenfluß von $Q_{0}=29 \mathrm{~m}^{3} / \mathrm{s}$ angenommen, da nach Albers und Christensen [1996] Plumes mit geringeren Volumen- bzw. Auftriebsflüssen wegen der starken Auskühlung unter der Lithosphäre keine Schmelzen mehr produzieren könnten, es sei denn, sie stammen nicht von der Kern-Mantelgrenze. Wegen den auftretenden, generell sehr großen Wellenlängen wird davon ausgegangen, daß Wellen in der Form wie sie in Experiment und Numerik beobachtet werden, allenfalls in Plumes existieren können, die eine Quellregion in $2900 \mathrm{~km}$ Tiefe haben. Eine Existenz in Plumes, die an der 660 km Diskontinuität entstehen, wird ausgeschlossen.

Betrachtet man einen schwachen Plume unter junger Lithosphäre (z.B. Galapagos-Plume, vergl. Sleep [1990]), so sind nach Tabelle 5.3 Temperaturanomalien von 5-7\% im Vergleich zum Plumekanal mit Wellenlängen von 400-600 km Länge verbunden. Da die Länge dieser Wellen nur ungefähr ein Fünftel des unteren Mantels ausmacht, kann hier ein von der thermischen Grenzschicht bzw. den Phasengrenzen im wesentlichen unbeeinflußter Aufstieg (zumindest im unteren Mantel) angenommen werden. Dadurch bleibt die Physik des Modells in einem solchen Fall mit der des realen Wellenaufstiegs vergleichbar. Nach Abbildung 6.1 könnte eine 7\%-ige Erhöhung einer $200 \mathrm{~K}$ heißen Plumetemperaturanomalie in $10 \mathrm{~km}$ Tiefe den Schmelzgrad von ca. $42 \%$ auf etwa $45 \%$ erhöhen, so daß auch in diesem Fall nicht mit einer signifikanten Erhöhung der vulkanischen Aktivität zu rechnen ist. Selbst bei noch 
größeren Wellen mit 12\%-igen Erhöhungen der Kanaltemperaturen ließe sich für dieses Beispiel unter junger Lithosphäre vermutlich kein signifikanter Unterschied im Vulkanismus feststellen.

Als weiteres Beispiel wird ein schwacher Plume $\left(Q_{0} \approx 30 \mathrm{~m}^{3} / \mathrm{s}\right.$, Temperaturanomalie des Kanals: $200 \mathrm{~K}$ ) unter alter und dicker Lithosphäre (ca. $100 \mathrm{~km}$ ) betrachtet, der keine Schmelzen produziert, weil seine Temperaturanomalie allein gerade nicht ausreicht die Soliduskurve in dieser Tiefe (vergl. Abbildung 6.1, Schnittpunkt Plumeadiabate / Solidus) zu überschreiten. Nach Tabelle $5.3 \mathrm{kann}$ eine große, etwa $800 \mathrm{~km}$ lange Welle durch eine etwa 1 Millionen Jahre andauernde Volumenflußerhöhung um den Faktor 1.5 die Temperaturanomalie des Kanals um 12\% erhöhen. Dadurch wäre es nach Abbildung 6.1 denkbar, daß der Solidus überschritten wird und sich eine 10-15\%-ige Aufschmelzung des Mantelgesteins ergibt. In diesem Fall wäre mit einer signifikanten Änderung der vulkanischen Aktivität für einen Zeitraum von etwa einer Million Jahren zu rechnen. Als Beispiele für Hotspots der Erde, bei dem der hier beschriebene Mechanismus im Zusammenhang mit einem schwachen Plume eine Rolle spielen könnte, kämen die Kanaren oder die Cap Verdischen Inseln in Frage. Beide Hotspots befinden sich in Regionen mit alter Lithosphäre $(>120 \mathrm{~km})$ und der nach Sleep [1990] angegebene Auftriebsfluß liegt bei $1000 \mathrm{~kg} / \mathrm{s}$ bzw. $1600 \mathrm{~kg} / \mathrm{s}$. Der Yellowstone-Hotspot gehört mit einem Auftriebsfluß von $1500 \mathrm{~kg} / \mathrm{s}$ und einer Lithosphärenmächtigkeit von etwa 80 km (Smith und Braile, [1994]) auch ungefähr zu dieser Kategorie. Dieser Hotspot war in seiner Vergangenheit sehr periodisch aktiv und hat eine Hotspot-Spur mit einer deutlichen Altersprogression hinterlassen (Smith und Braile, [1994]), was auf den Einfluß von Wellen hindeuten könnte. Allerdings ist es bei Hotspots unter kontinentaler Lithosphäre aufgrund der inhomogeneren Plattenstruktur um ein Vielfaches schwieriger, lokale Vorgänge, die zur Erhöhung der vulkanischen Aktivität führen könnten, auszuschließen. Da sich dieses Problem im geringerem Maße auch unter ozeanischer Lithosphäre stellt, wird es wahrscheinlich nicht gelingen, einen direkten Zusammenhang zwischen einer vulkanischen Aktivitätszunahme und einem vermuteten Wellenaufstieg herzustellen.

Aus den vorangegangenen Überlegungen heraus kann man zusammenfassend feststellen, daß die in dieser Arbeit untersuchten Wellenphänomene vermutlich nur in Plumes eine Rolle spielen könnten, deren Temperaturen unter der Lithosphäre gerade nicht den Solidus erreichen. Eine aufsteigende Welle wäre dann in der Lage, die nötige Temperaturanomalie zu liefern, um diesen zu überschreiten, so daß aus einem vulkanisch ruhigen Hotspot ein aktiver Hotspot werden kann. 


\section{Kapitel 7}

\section{Zusammenfassung}

Mit Hilfe experimenteller und numerischer Modellierungen wurde die Ausbreitung von wellenförmigen Volumenstörungen in thermischen Plumes untersucht. In Laborexperimenten wurden thermische Plumes durch Injizieren von heißem Sirup in eine mit kaltem Sirup gefüllte Plexiglassäule erzeugt. Dabei bildet sich zunächst ein Diapir aus, der angetrieben durch den thermischen Dichtekontrast langsam aufsteigt. Heißes Material strömt nach, so daß sich bei konstanter Injektionsrate nach einiger Zeit ein stationärer Kanal ausbildet. Durch eine kurzzeitige Erhöhung des injizierten Volumenstroms wurden Volumenstörungen erzeugt, die sich wellenartig im stationären Kanal ausbreiten.

Durch die Verwendung einer neuentwickelten berührungslosen Temperaturmeßmethode konnten radiale Temperaturprofile in stationären Plumekanälen und in darin aufsteigenden Wellen gemessen werden, ohne die Strömung zu beeinflussen. Die Meßmethode beruht auf einem die axialsymmetrische Struktur passierenden Laserstrahl, der durch die thermisch bedingten Variationen des Brechungsindexes abgelenkt wird. Zur Bestimmung der Temperaturen aus den Ablenkungsdaten wird ein der Seismologie entlehntes, modifiziertes WiechertHerglotz Inversionsverfahren verwendet.

Geschwindigkeitsmessungen wurden mit einer PIV (Particle Image Velocimetry) - Methode durchgeführt. Dabei wird die mit reflektierenden Teilchen versetzte Strömung mit einem Laserlichtschnitt beleuchtet. Durch Korrelation der zu unterschiedlichen Zeitpunkten aufgenommenen Partikelbilder lassen sich die Geschwindigkeiten in der Lichtschnittebene bestimmen.

Für die numerische Modellierung wurde ein zweidimensionales, axialsymmetrisches Finite-Differenzen-Programm entwickelt und auf seine korrekte Funktion hin getestet. Im Zusammenhang mit dieser Modellierung bestand durch die beiden experimentellen Meßmethoden zum ersten Mal die Möglichkeit, thermische Plumes und darin aufsteigende Wellen in Experiment und Numerik direkt quantitativ zu vergleichen. Dabei zeigte sich grundsätzlich eine gute bis sehr gute Übereinstimmung in den Plume- und Wellentemperaturen. Bei den Geschwindigkeitsmessungen stimmten die Werte im stationären Fall gut mit den numerisch bestimmten überein.

Experimentelle und numerische Studien zur Wellenstruktur haben gezeigt, daß insbeson- 
dere in niedrigen Aufstiegshöhen bzw. bei starken Hintergrundvolumenflüssen das thermische Erscheinungsbild der Wellen hauptsächlich durch eine thermische Verbreiterung des stationären Kanals und weniger durch eine Erhöhung der Maximaltemperatur gekennzeichnet ist. Dabei ist auch zu beobachten, daß sich im Zentrum der Welle ein annähernd konstanter Temperaturverlauf zeigt, der auf die interne Zirkulation in der Welle und die damit verbundene advektive Durchmischung zurückzuführen ist.

Mit zunehmender Aufstiegshöhe steigt die Temperaturanomalie der Welle an, da sie durch ihre schnelle Aufstiegsgeschwindigkeit weniger stark auskühlt als der Kanal.

Durch Detailbetrachtungen von gemessenen und berechneten Teilchengeschwindigkeiten innerhalb einer Welle konnte gezeigt werden, daß die radialen Geschwindigkeiten im oberen Bereich einer Welle stark lokalisiert und vom Betrage her größer sind als die Geschwindigkeiten im unteren Bereich der Welle. Dadurch wird Kanalmaterial von innen nach außen transportiert, was das Auftreten langgezogener vertikaler Temperaturanomalien hinter einer aufsteigenden Welle erklären konnte.

Aus Experimenten mit chemischen Plumes ist bekannt, daß eine große solitäre Welle eine kleine überholen kann. Bei den hier in diesem Zusammenhang durchgeführten Untersuchungen hat sich gezeigt, daß eine größere thermische Welle eine kleinere zwar einholen kann, dann aber ein Verschmelzen der Temperaturanomalien auftritt und sich die Wellen nicht mehr voneinander trennen.

Parameterstudien mit unterschiedlich großen Volumenstörungen in Plumekanälen mit unterschiedlichen Hintergrundvolumenflüssen haben gezeigt, daß ein systematischer Zusammenhang zwischen dem Wellenvolumen und der Geschwindigkeit sowie dem Volumen und der Temperaturanomalie besteht. Die dabei verwendete Skalierung wurde von Modellen für chemische Plumes übernommen. Sie lieferte insbesondere im Falle kleiner diffusiver Effekte konsistente Ergebnisse, so daß sie vor diesem Hintergrund auch für die Beschreibung von thermischen Wellen geeignet ist. Außerdem wurde deutlich, daß die Wellen eine bevorzugte Form und Ausbreitungsgeschwindigkeit haben, die nicht von der Dauer bzw. dem Faktor der Volumenflußerhöhung abhängen, sondern lediglich vom Volumeninhalt der Welle.

Numerische Rechnungen mit einer zusätzlich tiefenabhängigen Viskosität haben einen deutlichen Einfluß der Viskositätszunahme mit der Tiefe auf die radiale Ausdehnung des Plumes gezeigt. Ein nennenswerter Einfluß auf die Temperaturanomalien der Wellen konnte aber nicht beobachtet werden. Deshalb sind zur Vereinfachung die Parameterstudienergebnisse von Rechnungen mit rein thermischer Viskosität auf die Erde skaliert worden.

Dabei zeigte sich, daß die Länge von Wellen mit gleichen Erzeugungsmerkmalen bzw. gleichen dimensionslosen Volumina sehr stark mit dem Hintergrundvolumenfluß des Plumekanals zunimmt. Aus diesem Grund mußten Wellen mit großen Volumina, die in schwachen Plumes realistische Längen haben und große Temperaturanomalien hervorrufen, für starke Plumekanäle ausgeschlossen werden, da diese zum Teil Wellenlängen von deutlich mehr als 1000 km besitzen würden. Daher kommen für starke Plumes nur Wellen mit kleinen dimensionslosen Volumina in Frage, die nur geringe Temperaturerhöhungen im Plume verursachen, so daß sich der Aufschmelzungsgrad des Gesteins nur unwesentlich erhöht. 
Deshalb wurde bei Plumes mit Auftriebsflüssen von mehr als $2200 \mathrm{~kg} / \mathrm{s}$ (nach Ribe und Christensen [1999] der niedrigst mögliche Auftriebsfluß für den Hawaii-Plume) ein nennenswerter Einfluß der Wellen auf die Schmelzenproduktion ausgeschlossen.

Da bei der Skalierung auf schwache Plumes der Erde immer noch Mindestwellenlängen von $400 \mathrm{~km}$ aufgetreten sind, wurden Wellen, in der Form wie sie in Experiment und Numerik beobachtet wurden, für Plumes, die an der $660 \mathrm{~km}$ Diskontinuität entstehen, ebenfalls ausgeschlossen.

Nimmt man als Quellregion der für die Wellenausbreitung geeigneten Plumes die KernMantelgrenze an, so sind Wellen mit mehr als 1000 km Länge unwahrscheinlich. Bei der Betrachtung von Plumes mit niedrigen Volumenflüssen hat sich gezeigt, daß aber auch kürzere Wellen einen signifikanten Einfluß auf den Vulkanismus haben können, wenn sie unter alter und dicker Lithosphäre aufsteigen. Dabei ist es denkbar, daß ein Plumekanal den Solidus des Mantelgesteins gerade nicht oder nur geringfügig überschreitet. Eine aufsteigende Welle mit einer Länge von etwa 800 km könnte darin eine etwa 12\%-ige Erhöhung der Kanaltemperatur verursachen. Dadurch wird der Solidus deutlich überschritten, so daß eine 10-15\%-ige Aufschmelzung des Mantelgesteins auftreten kann.

Insgesamt hat sich gezeigt, daß der Einfluß der Wellen deutlich kleiner ist, als zunächst angenommen. Im Hinblick auf schwächere Plumes unter alter Lithosphäre kann er aber dennoch so groß sein, daß er als Ursache für einen periodisch erhöhten Vulkanismus nicht auszuschließen ist. 
Anhang 


\section{A.1 Sirupeigenschaften}

Der verwendete Glukosesirup trägt die Bezeichnung MOR-SWEET 01415². Der Hersteller macht folgende Angaben zu Eigenschaften und Zusammensetzung :

$\begin{array}{cc}\text { Trockensubstanz } & 79-80 \% \\ \text { Dichte bei } 20^{\circ} \mathrm{C} & \text { ca. } 1.42 \times 10^{3} \mathrm{~kg} / \mathrm{m}^{3} \\ \text { Sulfatasche } & \text { max. } 0.5 \% \\ \mathrm{pH}-\text { Wert } & 4.8-5.2 \\ \mathrm{SO}_{2} & \text { max. } 20 \mathrm{ppm} \\ \text { Brechungsindex bei } 20^{\circ} \mathrm{C} & 1.4944-1.4971 \\ \text { Viskosität bei } 20^{\circ} \mathrm{C} & 95 \mathrm{Pas} \\ \text { Viskosität bei } 40^{\circ} \mathrm{C} & 8 \mathrm{Pas} \\ \text { Viskosität bei } 60^{\circ} \mathrm{C} & 1.3 \mathrm{Pas} \\ & \\ \text { Zusammensetzung der Trockensubstanz: } \\ \text { D-Glukose } & \text { ca. } 7 \% \\ \text { Disaccharide (Maltose) } & \text { ca. } 45 \% \\ \text { Mehrfach- und höhermol. Saccharide ca. } 48 \%\end{array}$

\footnotetext{
${ }^{1}$ Der Sirup wurde von der Firma CERESTAR, Krefeld, freundlicherweise zur Verfügung gestellt.
} 


\section{A.1.1 Dichte, Viskosität und andere Größen}

In Abbildung A.1 ist die Temperaturabhängigkeit der Dichte dargestellt. Für den vom Hersteller angegebenen Wert von $1.42 \times 10^{3} \mathrm{~kg} / \mathrm{m}^{3}$ bei $20^{\circ} \mathrm{C}$ ergibt sich eine gute Übereinstimmung.

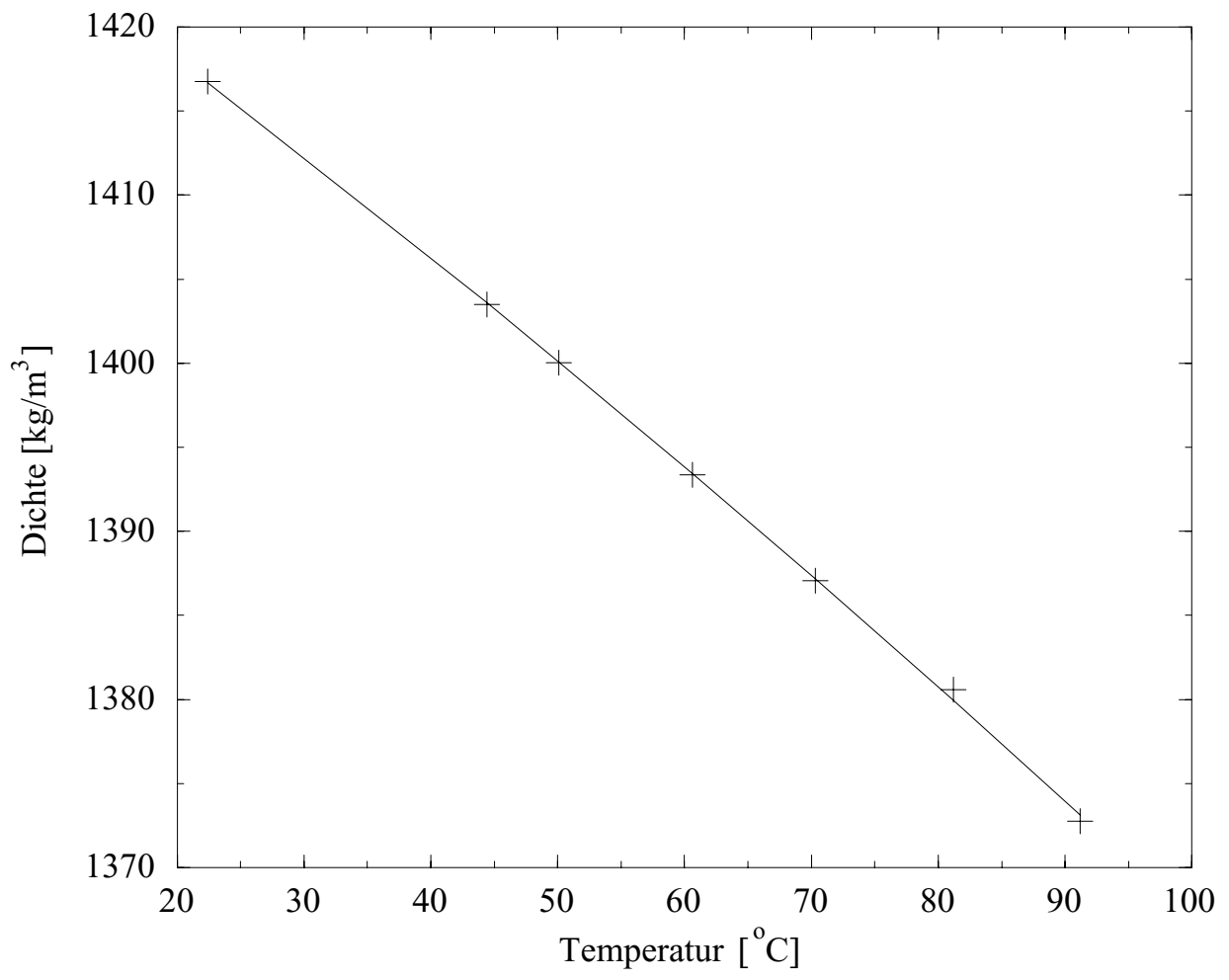

Abbildung A.1: Aufgetragen ist die Dichte in Abhängigkeit von der Temperatur.

Die quadratische Ausgleichsrechnung liefert folgende Temperaturabhängigkeit der Sirupdichte:

$$
\rho_{\text {Sirup }}=1429.2-0.540 T-8.163 \times 10^{-4} T^{2}\left[\mathrm{~kg} / \mathrm{m}^{3}\right]
$$


In Abbildung A.2 ist die Temperaturabhängigkeit der Sirupviskosität dargestellt. Diese ist mit einem Kugelfallviskosimeter nach dem Stokesschen Prinzip gemessen worden.

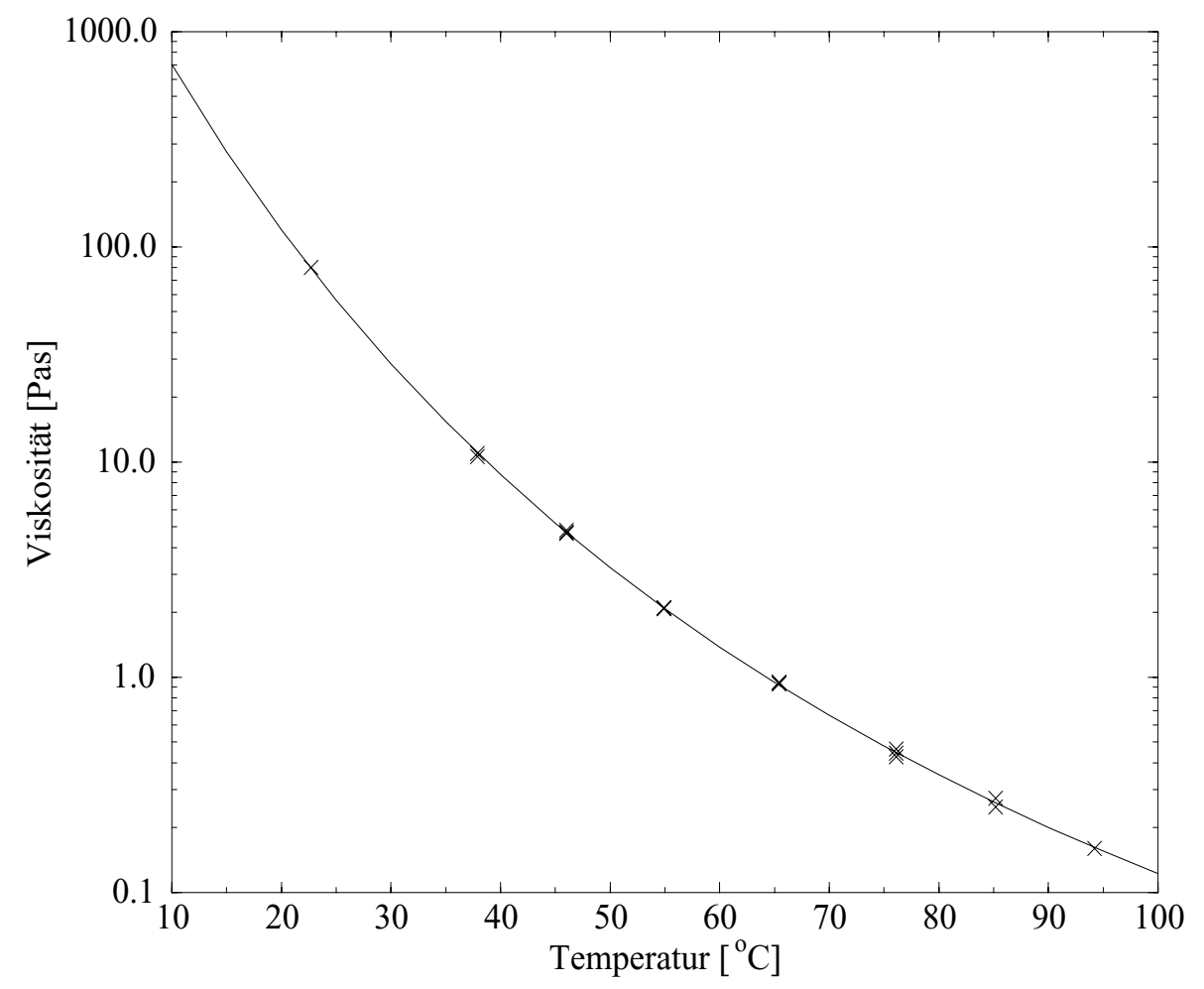

Abbildung A.2: Sirupviskosität in Abhängigkeit von der Temperatur.

Aus einer exponentiellen Ausgleichsrechnung ergeben sich folgende Koeffizienten für das Viskositätsgesetz:

$$
\begin{gathered}
\eta_{S}(T)=\eta_{\text {So }} \exp \left(\frac{1}{a+b T}\right) \\
\eta_{S o}=0.335 \times 10^{-4} \text { Pas } ; a=0.52 \times 10^{-1} ; b=7 \times 10^{-4} K^{-1}
\end{gathered}
$$

Damit variiert die Viskosität im experimentell relevanten Bereich zwischen $10^{\circ} \mathrm{C}$ und $100^{\circ} \mathrm{C}$ über vier Größenordnungen.

Weitere Eigenschaften des Sirups sind (Giannandrea, [1992]) :

$$
\text { Wärmeleitfähigkeit: } \quad \lambda=0.35+1.71 \times 10^{-4} T\left[\frac{\mathrm{W}}{\mathrm{mK}}\right]
$$

Wärmeausdehnungskoeffizient: $\quad \alpha_{v}=3.39 \times 10^{-4}+1.96 \times 10^{-6} T\left[K^{-1}\right]$

$$
\text { Spezifische Wärmekapazität: } \quad c_{p}=2202+3.935 T-6.6 \times 10^{-3} T^{2}\left[\frac{\mathrm{J}}{\mathrm{kgK}}\right]
$$




\section{A.1.2 Brechungsindex}

Der Zusammenhang zwischen Brechungsindex $n$ und Temperatur wird mit einem temperierbaren Abbe-Refraktometer gemessen.

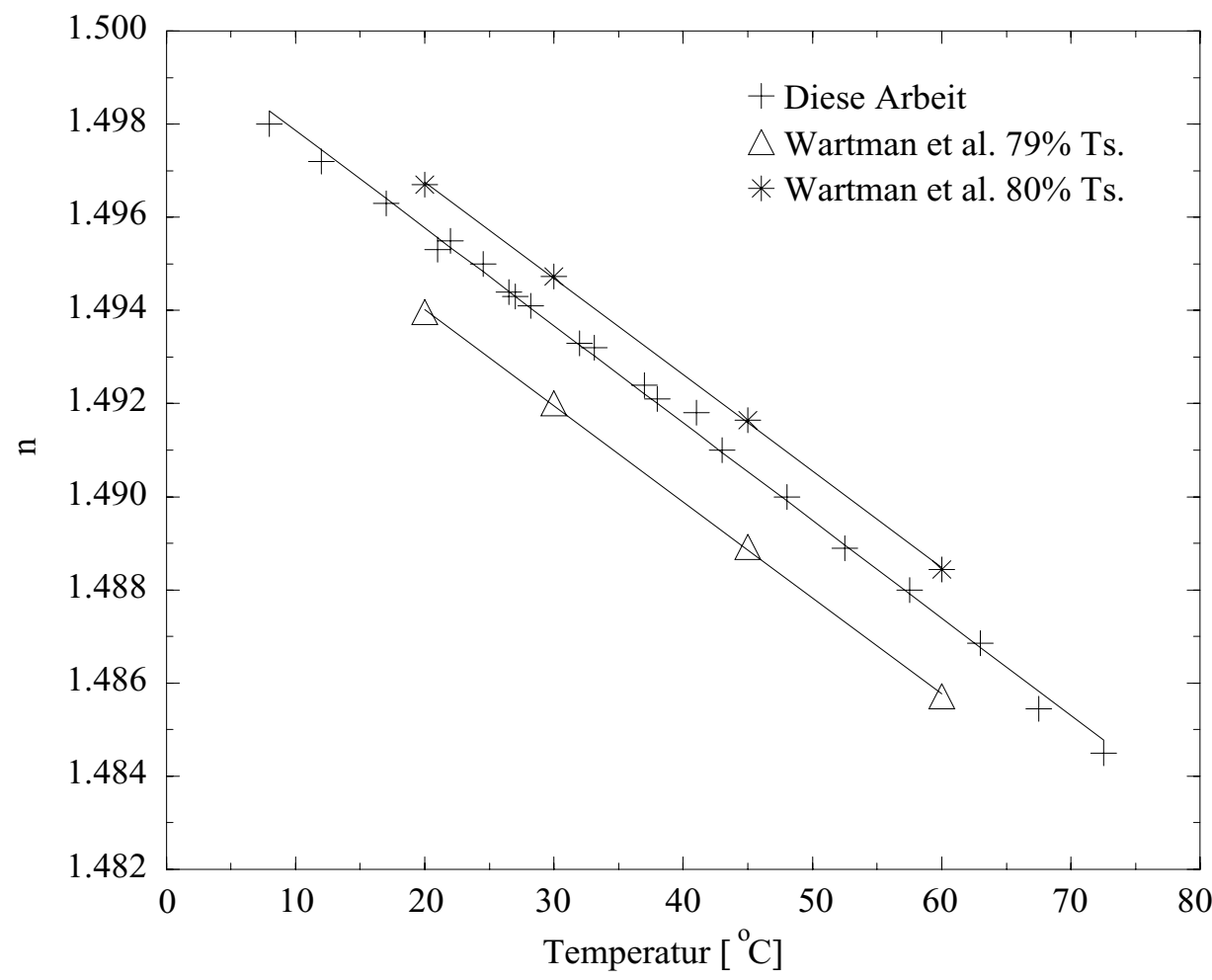

Abbildung A.3: Aufgetragen ist der Brechungsindex in Abhängigkeit von der Temperatur. Neben den gemessenen Daten finden sich auch zwei Geraden für $79 \%$ und 80\% Trockensubstanz(Ts.), die der Literatur (Wartman et al.) entnommen sind.

Der im Experiment verwendete Sirup hat laut Hersteller (Anhang A.1) eine Trockensubstanz(Ts.) von 79-80\%. Die für die thermische Abhängigkeit des Brechungsindexes gemessene Gerade liegt genau zwischen den von Wartman et al. bestimmten Geraden für $79 \%$ und 80\% Trockensubstanz.

Die Daten wurden durch folgende Funktionen angepaßt:

Diese Arbeit: $\quad \mathrm{n}=-2.0945 \times 10^{-4} T+1.5000$

Wartman et al. $79 \%$ Trockens.: $\quad \mathrm{n}=-2.0620 \times 10^{-4} T+1.4981$

Wartman et al. $80 \%$ Trockens.: $\mathrm{n}=-2.0671 \times 10^{-4} T+1.5009$ 


\section{A.2 Gleichungen}

\section{A.2.1 Stokesgleichung in Stromfunktionsformulierung}

Durch die Verwendung der Stromfunktionsformulierung ist die Kontinuitätsgleichung von vorn herein erfüllt, allerdings erhöht sich dadurch auch die Ordnung der Gleichung um eins. Sie lautet:

$$
\begin{array}{r}
\frac{\partial^{2}}{\partial z^{2}}\left(\frac{\eta}{r}\left[\frac{\partial^{2} \psi}{\partial r^{2}}-\frac{\partial^{2} \psi}{\partial z^{2}}-\frac{1}{r} \frac{\partial \psi}{\partial r}\right]\right)-\frac{\partial^{2}}{\partial r^{2}}\left(\frac{\eta}{r}\left[\frac{\partial^{2} \psi}{\partial r^{2}}-\frac{\partial^{2} \psi}{\partial z^{2}}-\frac{1}{r} \frac{\partial \psi}{\partial r}\right]\right)+ \\
\frac{\partial^{2}}{\partial r \partial z}\left(2 \eta\left[\frac{1}{r^{2}} \frac{\partial \psi}{\partial z}-\frac{2}{r} \frac{\partial^{2} \psi}{\partial r \partial z}\right]\right)+2 \frac{\partial}{\partial z}\left(\frac{\eta}{r^{2}}\left[\frac{2}{r} \frac{\partial \psi}{\partial z}-\frac{\partial^{2} \psi}{\partial r \partial z}\right]\right) \\
\frac{\partial}{\partial r}\left(\frac{\eta}{r^{2}}\left[\frac{\partial^{2} \psi}{\partial r^{2}}-\frac{\partial^{2} \psi}{\partial z^{2}}-\frac{1}{r} \frac{\partial \psi}{\partial r}\right]\right)-R a \frac{\partial}{\partial r} T=0
\end{array}
$$

\section{A.2.2 Diskretisierung der Wärmetransportgleichung}

Die Diskretisierung der Wärmetransportgleichung in Zylinderkoordinaten 3.28 ist hier exemplarisch für den ersten Teilschritt des verwendeten ADI-Verfahrens dargestellt. Dazu sind die radialen Ableitungen zusammen mit dem advektiven Term $u \cdot \partial T / \partial r$ implizit in $k+1 / 2$ und die vertikalen Ableitungen zusammen mit $w \cdot \partial T / \partial z$ explizit in $k$ genommen worden.

$$
\begin{gathered}
2\left(\frac{1}{h_{-}\left(h_{-}+h_{+}\right)} T_{i-1, j}^{k+1 / 2}-\frac{1}{h_{+} h_{-}} T_{i, j}^{k+1 / 2}+\frac{1}{h_{+}\left(h_{-}+h_{+}\right)} T_{i+1, j}^{k+1 / 2}\right) \\
+2\left(\frac{1}{k_{-}\left(k_{-}+k_{+}\right)} T_{i, j-1}^{k}-\frac{1}{k_{-} k_{+}} T_{i, j}^{k}+\frac{1}{k_{+}\left(k_{-}+k_{+}\right)} T_{i, j+1}^{k}\right) \\
\quad+\frac{1}{2 r}\left(\frac{1}{h_{+}}\left(T_{i+1, j}^{k+1 / 2}-T_{i, j}^{k+1 / 2}\right)+\frac{1}{h_{-}}\left(T_{i, j}^{k+1 / 2}-T_{i-1, j}^{k+1 / 2}\right)\right) \\
-u_{i, j}\left(\frac{1}{2 h_{+}}\left(T_{i+1, j}^{k+1 / 2}-T_{i, j}^{k+1 / 2}\right)+\frac{1}{2 h_{-}}\left(T_{i, j}^{k+1 / 2}-T_{i-1, j}^{k+1 / 2}\right)\right) \\
-w_{i, j}\left(\frac{1}{2 k_{+}}\left(T_{i, j+1}^{k}-T_{i, j}^{k}\right)+\frac{1}{2 k_{-}}\left(T_{i, j}^{k}-T_{i, j-1}^{k}\right)\right) \\
=2 \frac{T_{i j}^{k+1 / 2}-T_{i j}^{k}}{\Delta t}
\end{gathered}
$$




\section{A.3 Verwendete Skalen}

Die hier aufgeführten Skalen, die im Zusammenhang mit den in Kapitel 5.1 dargestellten Parameterstudien ${ }^{1}$ verwendet werden, sind in dieser Form Olson und Christensen [1996] entnommen worden. Sie wurden von den Autoren im Zusammenhang mit einer in eine hochviskose Umgebung eingebettete, niedrigviskose Kanalströmung, die sich näherungsweise als Poiseuillesche Rohrströmung beschreiben läßt, gewählt (vergl. Abbildung A.4). Sie lauten:

$$
\begin{aligned}
\text { Längenskala }: L & =\left(\frac{\eta_{M} A_{0}}{8 \pi \eta_{L}}\right)^{1 / 2}=\frac{Q_{0}^{1 / 4} \eta_{M}^{1 / 2}}{\left(8 \pi \eta_{L} \Delta \rho g\right)^{1 / 4}} \\
\text { Zeitskala: } T & =\frac{1}{\Delta \rho g}\left(\frac{8 \pi \eta_{L} \eta_{M}}{A_{0}}\right)^{1 / 2}=\frac{\left(8 \pi \eta_{L}\right)^{1 / 4} \eta_{M}^{1 / 2}}{(\Delta \rho g)^{3 / 4} Q_{0}^{1 / 4}}
\end{aligned}
$$

Geschwindigkeitsskala: $\frac{L}{T}=\frac{Q_{0}^{1 / 2}(\Delta \rho g)^{1 / 2}}{\left(8 \pi \eta_{L}\right)^{1 / 2}}=\left(\frac{\Delta \rho g Q_{0}}{8 \pi \eta_{L}}\right)^{1 / 2}$

$$
\text { Volumenskala: } V_{0}=Q_{0} T=A_{0} L=\frac{\eta_{M}^{1 / 2} A_{0}^{3 / 2}}{\left(8 \pi \eta_{L}\right)^{1 / 2}}=\frac{Q_{0}^{3 / 4} \eta_{M}^{1 / 2}\left(8 \pi \eta_{L}\right)^{1 / 4}}{(\Delta \rho g)^{3 / 4}}
$$

Volumenflußskala: $Q_{0}$

\footnotetext{
${ }^{1}$ Bei der systematischen Untersuchung der Wellentemperaturen bzw. Anomalien ist abweichend von dem hier aufgeführtem Ausdruck die diffusive Längenskala $Q / \kappa$ verwendet worden.
}

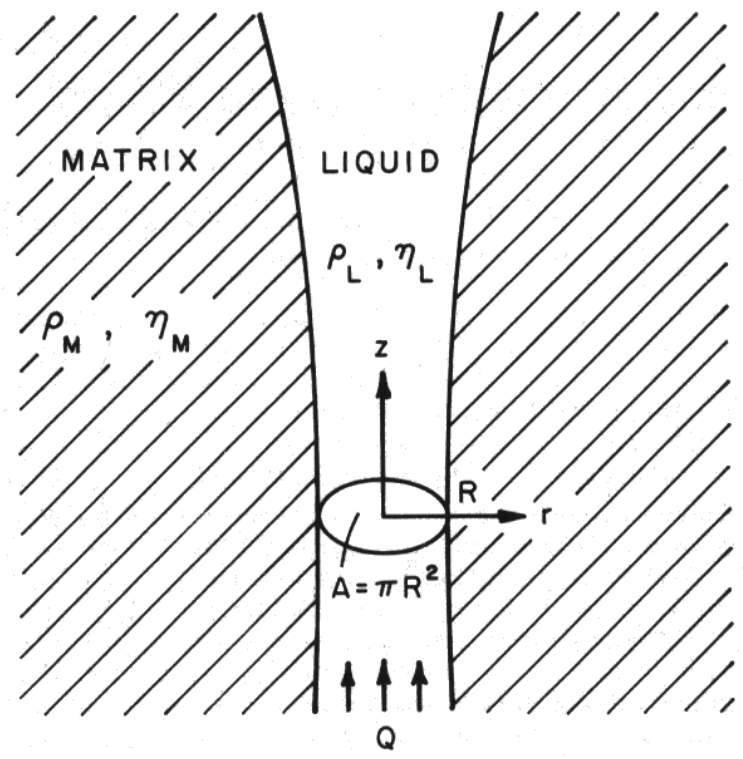

Abbildung A.4: Skizze der niedrigviskosen, kanalartigen Strömung in einer hochviskosen Matrix (aus Olson und Christensen [1986]). 
Dabei ist $\Delta \rho$ der Dichtekonstrast zwischen $\rho_{M}$ (Dichte der Matrixflüssigkeit) und $\rho_{L}$ (Dichte der Kanalflüssigkeit). $\eta_{L}$ bzw. $\eta_{M}$ stehen für die Viskositäten der Kanal- bzw. der Matrixflüssigkeit. $Q_{0}$ ist der ungestörte Volumenfluß, der bei einer Poiseuilleschen Rohrströmung durch

$$
Q_{0}=\frac{\Delta \rho g}{8 \pi \eta_{L}} A_{0}^{2}
$$

mit der Querschnittsfläche $A_{0}$ verbunden ist. 


\section{A.4 Verwendete Geräte}

\section{A.4.1 Nd:YAG-Laser}

Für ein Großteil der PIV-Messungen ist ein durch Blitzlampen gepumter Nd:YAG-Laser der Firma Quantel vom Typ „ULTRA CFR“ verwendet worden. Technische Daten:

- Wellenlänge: $532 \mathrm{~nm}$

- Energie pro Puls: $30 \mathrm{~mJ}$

- Pulsrate: $20 \mathrm{~Hz}$

- Strahldurchmesser: $2.8 \mathrm{~mm}$

- Strahldivergenz: $<8 \mathrm{mrad}$

- Vertikal polarisiert

Zur Erzeugung eines etwa $1 \mathrm{~mm}$ dicken Laserlichtschnitts sind verschiedene Linsen des „Microbench“-Systems der Firma Spindler\&Hoyer verwendet worden. Detailierte Beschreibungen zur Erzeugung eines Laserlichtschnitts finden sich zum Beispiel in Raffel et al. [1998].

\section{A.4.2 CCD-Kamera für PIV}

Zur Aufnahme digitaler PIV-Bilder ist das „PixelFly Scientific“-Kamerasystem der Firma $\mathrm{PCO}^{1}$, Computer Optics, Kelheim verwendet worden. Technische Daten:

- Schwarz/weiß-Kamera mit 1280(H) $\times 1024(V)$ Pixeln auf 2/3“ Sensor

- 12 bit dynamische Auflösung

- Bildrate bei voller Auflösung: 12.5 fps (Frames per s)

\section{A.4.3 HeNe-Laser für Temperaturmeßmethode}

Der verwendete HeNe-Laser mit der Leistung von $10 \mathrm{~mW}$ ist ein Produkt der Firma SpectraPhysics mit der Bezeichnung 105-1.

Die Austrittsstrahltaille hat einen Querschnittsdurchmesser von $0.8 \mathrm{~mm}$.

\section{A.4.4 CCD-Kamera für Temperaturmeßmethode}

Die für die Temperautrmeßmethode verwendete Zeilenkamera der Firma „Focus GmbH“, Darmstadt trägt die Bezeichnung CCD 1000 und arbeitet mit einem 2048 Pixel-Sensor der Firma Sony.

${ }^{1}$ Mein Dank gilt Herrn Kaspar der Firma PCO, der mir ein vollständiges Kamerasystem für meine Messungen zur Verfügung gestellt hat. 


\section{A.4.5 Galvanoscanner}

Bei dem für die Temperaturmeßmethode verwendeten Galvanoscanner handelt es sich um ein Produkt der Firma GENERAL SCANNING INC. mit der Bezeichnung G325DT und der Steuerelektronik AE 850. Die Stromversorgung erfolgt über ein Standardnetzteil der Firma Rohde \& Schwartz. Die Steuerspannung wird von einem Frequenzgenerator der Firma Hewlett Packard 3325B Synthesizer / Funktion Generator geliefert. 


\section{Literaturverzeichnis}

Albers, M., Numerische Untersuchung stationärer Mantel-Plumes, Diplomarbeit, Institut für Geophysik, Universität Göttingen (1995).

Albers, M. und U.R. Christensen, The excess temperature of plumes rising from the coremantle boundary, Geophys. Res. Lett. 23, 3567-3570 (1996).

Albers, M., Numerische Untersuchungen zur Wechselwirkung von Mantelplumes mit Mittelozeanischen Rücken, Dissertation, Institut für Geophysik, Universität Göttingen (2000).

Batchelor, G.K., An introduction to fluid dynamics, Cambridge University Press, Cambridge (1992).

Bunge, H.-P., M.A. Richards und J.R. Baumgardner, Effect of depth-dependent viscosity on the planform of mantle convection, Nature 379, 436-438 (1996).

Čadek, O. und L. Fleitout, A global model with imposed plate velocities and partial layering, J. Geophys. Res. 104, 29055-29075 (1999).

Christensen, U.R., Effects of phase transitions on mantle convection, Ann.Rev.Earth Planet.Sci., 23, 65-87, (1995).

Christensen, U.R., Influence of chemical buoyancy on the dynamics of slabs in the transition zone, J. Geophys. Res. 102, 22435-22443 (1997).

Christensen, U.R. und H. Harder, 3-D convection with variable viscosity, Geophys. J. Int. 104, 213-226 (1991).

Davaille, A., Simultaneous generation of hotspots and superswells by convection in a heterogeneous planetary mantle, Nature 402, 756-760 (1999).

Duncan, R.A. und M.A. Richards, Hotspots, mantle plumes, flood basalts, and true polar wander, Rev. Geophys. 29, 31-50 (1991).

Eckelmann, H., Einführung in die Strömungsmeßtechnik, Teubner, Leitfäden der angewandten Mathematik und Mechanik, Bd. 74, Stuttgart (1997). 
Fowler, C.M.R., The solid earth, Cambridge University Press, Cambridge (1990).

Frigo, M. und S.T. Johnson, www.fftw.org, fftw version 2.1.3, Massachusetts Institute of Technology, anonymous ftp to fftw.org.

Giannandrea, E.R., Experimentelle Simulation der Konvektionsströme im Erdmantel, Dissertation, Fachbereich Geowissenschaften der Johannes-Gutenberg-Universität in Mainz (1992).

Griffiths, R.W., The differing effects of compositional and thermal buoyancies on the evolution of mantle diapirs, Earth Planet. Sci. Lett. 43, 261-273 (1986).

Griffiths, R.W. und I.H. Campbell, Stirring and structure in mantle starting plumes, Earth Planet. Sci. Lett. 99, 66-78 (1990).

Hauri, E.H., J.A. Whitehead und S.R. Hart, Fluid dynamic and geochemical aspects of entrainment in mantle plumes, J. Geophys. Res. 99, 24275-24300 (1994).

Herbert, V. und O. LeContel, Origine et structuré thermique des panaches, MSc thesis, Ecole Normale Supérieure de Paris (1991).

Jarvis, G.T. und D.P. McKenzie, Convection in a compressible fluid with infinite Prandtl number, J. Fluid Mech. 96, 515-583 (1980).

Kaufmann, G. und K. Lambeck, Mantle dynamics, postglacial rebound and the radial viscosity profile, Phys. Earth Planet. Int. 121 (3-4), 303-327 (2000).

Kellogg, L.H., B.H. Hager und R.D. van der Hilst, Compositional stratification in the deep mantle, Science 283, 1881-1884 (1999).

Lambeck, K., C. Smither und P. Johnston, Sea-level change, glacial rebound and mantle viscosity for northern Europe, Geophys. J. Int. 134, 102-144 (1998).

Laudenbach, N., Entwicklung einer berührungslosen Temperaturmeßmethode zur Untersuchung experimentell erzeugter thermischer Plumes, Diplomarbeit, Institut für Geophysik, Universität Göttingen (1997).

Laudenbach, N. und U.R. Christensen, An optical method for measuring temperature in laboratory models of mantle plumes, Geophys. J. Int. 145, 528-534 (2001).

Lay, T. und Wallace, T.C., Modern Global Seismology, Academic Press, San Diego (1995).

Li, X., R. Kind, K. Priestley, S.V. Sobolev, F. Tilmann, X. Yuan und M. Weber, Mapping the Hawaiian plume conduit with converted seismic waves, Nature 405, 938-941 (2000).

McKenzie, D. und M. Bickle, The volume and composition of melt generated by extension of the lithosphere, J. Petrol. 29, 625-679 (1988). 
Morgan, W.J., Plate motions and deep mantle convection, Mem. Geol. Soc. Am. 132, 7-22 (1972).

Nataf, H. C., C. Froidevaux, J.L. Levrat und M. Rabinowicz, Laboratory convection experiments: Effect of lateral cooling and generation of instabilities in the horizontal boundary layers, J. Geophys. Res. 86, 6143-6154 (1981).

Olson, P., Hot spots, swells and mantle plumes, in: M.P. Ryan (Hrsg.), Magma transport and storage, Wiley, New York (1990), p. 33-51.

Olson, P. und U.R. Christensen, Solitary wave propagation in a fluid conduit within a viscous matrix, J. Geophys. Res. 91, 6367-6374 (1986).

Olson, P., G. Schubert und C. Anderson, Structure of axisymmetric mantle plumes, J. Geophys. Res. 98, 6829-6844 (1993).

Olson, P., G. Schubert, C. Anderson und P. Goldman, Plume formation and lithosphere erosion: A comparison of laboratory and numerical experiments, J. Geophys. Res. 93, 15065-15084 (1988).

Olson, P. und H. Singer, Creeping plumes, J. Fluid Mech. 158, 511-531 (1985).

Press, W.A., S.A. Teukolsky, W.T. Vetterling und B.P. Flannery, Numerical Recipes in C, Cambridge Univ. Press, (1992).

Raffel, M, C. Willert und J. Kompenhans, Particle Image Velocimetry, a practical guide, Springer, Berlin Heidelberg (1998).

Ribe, N.M. und U.R. Christensen, The dynamical origin of Hawaiian volcanism, Earth Planet. Sci. Lett. 171, 517-531 (1999).

Ribe, N.M., U.R. Christensen und J. Theißing, The dynamics of plume-ridge interaction, 1: Ridge-centered plumes, Earth Planet. Sci. Lett. 134, 155-168 (1995).

Ritter, J.R.R., M. Jordan, U.R. Christensen und U. Achauer, A mantle plume below the Eifel volcanic fields, Earth Planet. Sci. Lett. 186, 7-14 (2001).

Ronneberger, O., M. Raffel und J. Kompenhans, Advanced evaluation algorithms for standard and dual plane particle image velocimetry, Proceedings 9th International Symposium on Application of Laser Techniques to Fluid Mechanics, Lisbon paper 10.1, (1988).

Schowalter, W. R., Mechanics of non-Newtonian fluids, Pergamon Press, Oxford, (1978).

Schubert, G., P. Olson, C. Anderson und P. Goldman, Solitary waves in mantle plumes, J. Geophys. Res. 94, 9523-9532 (1989). 
Shen, Y., S.C. Solomon, I.T. Bjarnason und C.J. Wolfe, Seismic evidence for a lower-mantle origin of the Iceland plume, Nature 395, 62-65 (1998).

Sleep, N.H., Hotspots and mantle plumes: Some phenomenology, J. Geophys. Res. 95, 67156736 (1990).

Smith, R.B. und L.W. Braile, The Yellowstone hotspot, J. Volcanology and Geotherm. Rese$\operatorname{arch~61,~121-187~(1994).~}$

Turcotte, D.L. und G. Schubert, Geodynamics, Wiley, New York (1982).

Wartman, A.M., C. Hagberg und M.A. Eliason, Refractive index - Dry substance relationships for comercial corn syrups, Augustana Research Foundation, Rock Island, Illinois.

Watson, S. und D. McKenzie, Melt generation by plumes: A study of Hawaiian volcanism, J. Petrol. 32, 501-537 (1991).

Westerweel, J., Efficient detection of spurious vectors in particle image velocimetry data, Exp. i. Fluids 16, 236-247 (1994).

Westerweel, J., D. Dabiri und M. Gharib, The effect of a discrete window offset on the accuracy of cross-correlation analysis of digital PIV recordings, Exp. i. Fluids 23, 2028 (1997).

White, R.S., Melt production rates in mantle plumes, Phil. Trans. R. Soc. Lond. A 342, 137153 (1993).

Whitehead, J.A. und K.R. Helfrich, Wave transport of deep mantle material, Nature 336, 59-61 (1988).

Whitehead, J.A. und K.R. Helfrich, Magma waves and diapiric dynamics, in: M.P. Ryan (Hrsg.), Magma transport and storage, Wiley, New York (1990), p. 53-76.

Wolfe, C.J., I.T. Bjarnason, J.C. VanDecar und S.C. Solomon, Seismic structure of the Iceland mantle plume, Nature 385, 245-247 (1997). 


\section{Danksagung}

An erster Stelle danke ich Herrn Prof. Dr. Christensen für die gute Betreuung und die Unterstützung, die ich während der Anfertigung dieser Arbeit erhalten habe. Die Labor- und die umfangreiche Computerausstattung des Instituts haben die Arbeit nicht nur sehr erleichtert, sondern zum Teil überhaupt erst möglich gemacht. Prof. Christensen gilt mein Dank, da er mich in meinen Vorhaben bestärkt hat und mir die Gelegenheit gab, meine Ergebnisse auf vielen nationalen und internationalen Tagungen zu präsentieren.

Herrn Prof. Dr. Eckelmann vom Institut für Nichtlineare Dynamik der Universität Göttigen danke ich sehr für die Übernahme des Korreferats und für das umfassende Interesse am Fortgang dieser Arbeit.

Ein großer Dank geht auch an die Mitarbeiterinnen und Mitarbeiter der Geodynamik Arbeitsgruppe, die nicht nur nach ,5-Minuten-Beiträgen“ gerne mit mir über meine Ergebnisse diskutiert, sondern jederzeit meine Arbeit durch Fragen und Anregungen bereichert haben. Bei Michael Albers und Johannes Wicht bedanke ich mich sehr herzlich für die sorgfältige Durchsicht verschiedenster Teile dieser Arbeit.

Wilfried Steinhoff und Rudi Deppe aus der Feinmechanischen Werkstatt gilt mein Dank für das Anfertigen des Plumeexperiments und das Lösen von unzähligen „kleinen und großen Problemen“, die während der experimentellen Arbeit (meistens unerwartet) angefallen sind.

Bei Matthias Wehmeier bedanke ich mich für prozessorgesteuerte Geräte zum automatischen Auslösen von Spiegelreflexkameras und die umfangreiche Beratung hinsichtlich chemischer Fotographie, die im Zusammenhang mit ersten PIV-Experimenten von großer Bedeutung war.

Ulrich Einecke danke ich für die Betreuung des Rechnernetzes und sein Verständnis dafür, daß beim zeitabhängigen Rechnen in der „Hohen_Box“ größere Datenmengen anfallen können.

Nicht nur der Großteil der finanziellen, sondern auch ein Teil der wissenschaftlichen Unterstützung kam vom Graduiertenkolleg für Strömungsinstabilitäten und Turbulenz der Universität Göttingen. Ohne den über das Graduiertenkolleg zustande gekommenen Kontakt zur DLR Göttingen wären PIV-Messungen in dieser Arbeit nur schwer möglich gewesen. Danke an Prof. Eckelmann, Andreas Schröder und die DLR Göttingen für die Vermittlung bzw. das Ausleihen eines ,gepumpten“ Lasers!

Bei Frau Eggert-Heise, Frau Schliebe und Frau Eberhart bedanke ich mich für das Bearbeiten etlicher Dienstreiseanträge bzw. von Abrechnungen und Hiwi-Verträgen.

Mein größter Dank gilt meinen Eltern, die mir das Studium ermöglicht und mir immer einen großen Rückhalt gegeben haben. Meinem Vater und Katja danke ich für das vollständige Korrekturlesen der Arbeit und hoffe, daß es nicht zu anstrengend war. Katja danke ich

außerdem für ihr Verständnis während der Zeit des Zusammenschreibens, sowie für die vielen aufmunternden Anrufe und Besuche! 



\section{Lebenslauf:}

\section{Persönliche Daten}

Name:

Geburtsdatum:

Geburtsort:

Staatsangehörigkeit:

\section{Schulbildung}

07/1977-06/1981

07/1981-06/1983

07/1983-06/1987

07/1987-06/1990

\section{Zivildienst}

07/1990-10/1991

\section{Studium}

ab 10/1991

$10 / 1993$

ab 10/1993

10/1995-04/1997

11/1996-04/1997

07/1997

08/1997-10/2000

11/2000-10/2001

08/1997-10/2001

\section{Auszeichnungen}

03/1999

\section{Eingeladener Vortrag}

$11 / 2000$
Nils Laudenbach

$$
\text { 1. November } 1970
$$

Bremen

Deutscher

Grundschule an der Admiralstraße, Bremen

Orientierungsstufe SZ Regensburger Straße, Bremen

Gymnasialer Zweig SZ Regensburger Straße

Gymnasiale Oberstufe, Lange Reihe, Bremen, Abschluß: Abitur
Grundstudium Physik / Geophysik an der Georg-August-Universität Göttingen

Vordiplom Physik

Hauptstudium Physik an der Universität Göttingen, Spezialgebiet Geophysik

Diplomarbeit am Institut für Geophysik der Universität Göttingen in der Arbeitsgruppe von Prof. Dr. U. Christensen. Thema der Arbeit:,Entwicklung einer berührungslosen Temperaturmeßmethode zur Untersuchung experimentell erzeugter thermischer Plumes."

Studentische Hilfskraft am Institut für Geophysik, finanziert aus Drittmitteln der Deutschen Forschungsgemeinschaft.

Diplomprüfung Physik

Stipendiat im Graduiertenkolleg für Strömungsinstabilitäten und Turbulenz und wissenschaftliche Hilfskraft am Institut für Geophysik, finanziert aus Drittmitteln der Deutschen Forschungsgemeinschaft.

Wissenschaftlicher Mitarbeiter am Institut für Geophysik.

Im Rahmen der genannten Finanzierungen bestand Gelegenheit zur Promotion. Thema: „Experimentelle und numerische Untersuchungen zur Ausbreitung von Volumenstörungen in thermischen Plumes.“

Vortragsauszeichnung durch die Deutsche Geophysikalische Gesellschaft anläßlich ihrer 59. Jahrestagung in Braunschweig.

Vortrag am Institut für Meteorologie und Geophysik der Johann Wolfgang Goethe-Universität, Frankfurt am Main, Titel: „Experimentelle und numerische Modellierung von thermischen Plumes“. 